\title{
Rotor Inflow Noise Caused by a Boundary Layer: Inflow Measurements and Noise Predictions
}

\author{
Michael Andrew Morton
}

Thesis submitted to the faculty of the Virginia Polytechnic Institute and State University in partial fulfillment of the requirements for the degree of

\author{
Master of Science \\ in \\ Aerospace Engineering
}

\author{
William J. Devenport \\ Stewart A. L. Glegg \\ Kevin T. Lowe \\ $08 / 02 / 2012$ \\ Blacksburg, Virginia
}

Keywords: Turbulent boundary layer, haystacking, velocity correlation, turbulence ingestion, rotor noise 


\title{
Rotor Inflow Noise Caused by a Boundary Layer: Inflow Measurements and Noise Predictions
}

\author{
Michael Andrew Morton
}

\begin{abstract}
A rotor immersed in a thick turbulent boundary layer produces unsteady loading on the blades which generates unwanted noise and vibration. Two point velocity fluctuations were measured in detail to determine the full four-dimensional correlation function of a boundary layer generated over a smooth wall in the Virginia Tech Stability Wind Tunnel. The correlation function reveals anisotropy in the flow dominated by a large scale correlation structure elongated in the streamwise direction and inclined relative to the wall. This correlation function was then evaluated in the blade frame of reference of an idealized 10 bladed rotor partially immersed in the flow. Blade to blade upwash coherence shows significant asymmetry which is a direct result of the anisotropy of the flow. Using a newly developed theory, the correlation function was used to predict the far-field radiated noise from the rotor at various operating and flow conditions. Predictions show the sound field is dominated by the effects of "haystacking" which is further increased with the inclusion of the presence of the wall. Directivity predictions suggest the far-field sound field acts like a monopole/dipole combination.
\end{abstract}




\section{Acknowledgements}

I would like to start off by thanking my parents for getting me into the position to accomplish all of this. Without their love and support none of this would have been possible.

Next, I would like to thank my advisor Dr. William Devenport. I have learned a great deal from him over the last two years and am very grateful to be a part of his research group. He has encouraged me to become a better researcher, student, and person. I couldn't have asked for a better mentor.

I would also like to thank my committee members Dr. Stewart Glegg and Dr. Todd Lowe for their assistance throughout my research.

I would like to thank Bill Oetjens for his assistance in the wind tunnel and understanding when things didn't go as planned. Also, I thank the workers in the AOE machine shop who were available to help when things went awry keeping things on schedule.

As for my coworkers in lab 7, I would like to thank you all for your help during my time at Virginia Tech. Thanks to Dr. Nathan Alexander, Jon Forest, Manuj Awasthi, Tim Meyers, Chris Rock, and Matt Rasnick for their help working in the wind tunnel especially during the installation of the wall. Special thanks go to Dr. Aurelien Borgoltz for spending the long overnight hours in the tunnel taking data as well as sharing his amazing knowledge of quadwire repair. They have all been great friends and made my experience in Blacksburg enjoyable.

Lastly, I would like to thank the Office of Naval Research for their funding of this project under grant N00014-10-1-0908, making this research possible.

Again, thank you all.

Michael Morton 
CHAPTER 1 INTRODUCTION

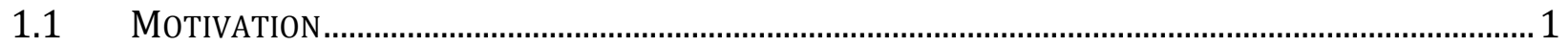

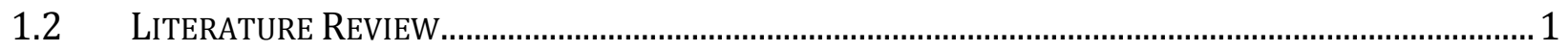

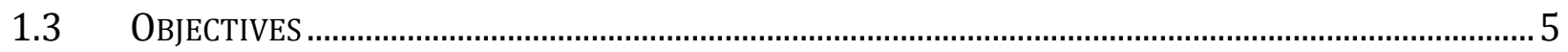

CHAPTER 2 APPARATUS AND INSTRUMENTATION.................................................... 6

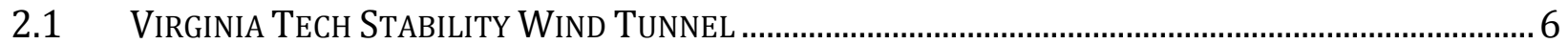

2.2 TESTING SURFACE AND INSTRUMENTATION ……………........................................................... 7

2.2.1 False Wall .......................................................................................................................

2.2.2 Coordinate System ……....................................................................................................11

2.2.3 Reference Instrumentation ….....................................................................................12

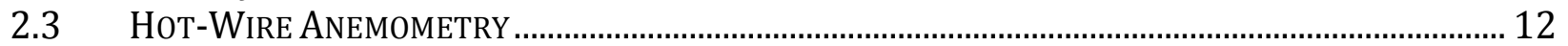

2.3.1 Hot-Wire Probes.........................................................................................................12

2.3.2 Constant Temperature Anemometers ............................................................................... 13

2.3.3 Quad-wire Calibration.................................................................................................13

2.4 PRoBe Mounting AND Positioning ………………..................................................................... 13

2.4.1 Traverse System ............................................................................................................... 13

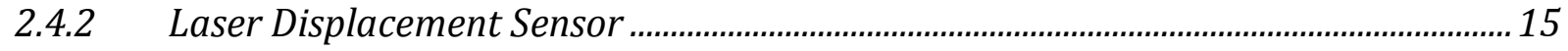

2.4.3 Probe Separation ............................................................................................................. 16

2.5 Two-PoINT QUADWIRE MEASUREMENT GRIDS.............................................................................. 17

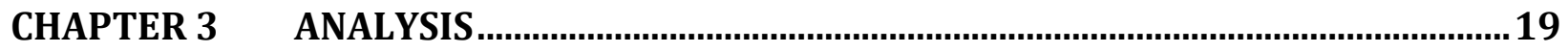

3.1 MEASUREMENT SUMMARY ................................................................................................... 19

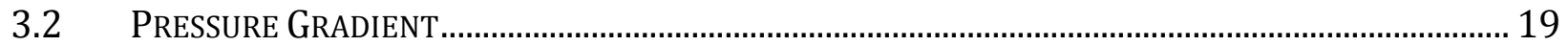

3.3 SINGLE PoINT MEASUREMENTS ................................................................................................ 20

3.3.1 Mean Velocities........................................................................................................... 20

3.3.2 Boundary Layer Turbulence....................................................................................24

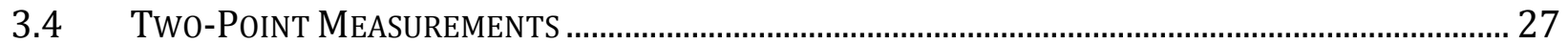

3.4.1 Probe Angle Transformation ....................................................................................28

3.4.2 Two-Point Velocity Correlation …………….........................................................................29

3.4.3 Proper Orthogonal Decomposition and Linear Stochastic Estimation ........................ 37

3.4.4 Compact Eddy Structures...............................................................................................40

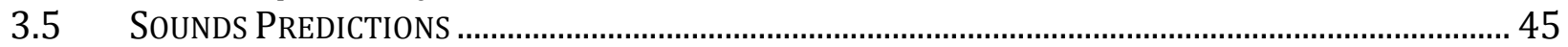

3.5.1 Sound Prediction Methodology ………….............................................................................. 45

3.5.2 Rotor Geometry ...............................................................................................................................4 4

3.5.3 Source Terms as Seen in the Blade Reference Frame ...................................................... 48

3.5.4 Upwash Coherence ..................................................................................................... 50

3.5.5 Farfield Sound...................................................................................................................... 54

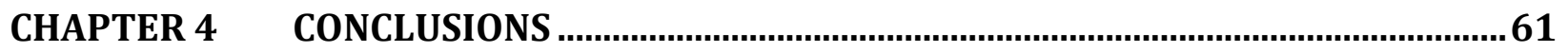

REFERENCES 


\section{Nomenclature}

\section{Roman}

\begin{tabular}{|c|c|}
\hline$A_{i j}$ & Estimation coefficients \\
\hline$B$ & Number of total rotor blades \\
\hline$c$ & Speed of sound; also, blade chord \\
\hline$C_{f}$ & Skin friction coefficient \\
\hline$f$ & Frequency $(\mathrm{Hz})$ \\
\hline$H$ & Boundary layer shape factor; also, Hankel function \\
\hline$h$ & Rotor hub height \\
\hline$J$ & Advance ratio \\
\hline$K$ & Fourier series coefficient \\
\hline$k$ & Blade index number \\
\hline$m$ & Bessel function order \\
\hline$M_{r}$ & Mach number of the source velocity \\
\hline$n$ & Blade index number; also, mode number; also blade normal vector \\
\hline$p$ & Difference in blade indices, $(p=n-k)$ \\
\hline$p^{\prime}$ & Acoustic pressure \\
\hline$R$ & Rotor radius; also, blade radial location \\
\hline$R_{e}$ & Reynolds number \\
\hline$r$ & Distance from source to observer \\
\hline$r_{o}$ & Distance from hub to observer \\
\hline$R_{i j}$ & Space-time correlation function \\
\hline$S$ & Sears function \\
\hline$S_{L L}$ & Unsteady lift spectrum \\
\hline$S_{p p}$ & Unsteady power spectrum \\
\hline$S_{w w}$ & Unsteady upwash spectrum \\
\hline$T$ & Time Period \\
\hline$t$ & Time \\
\hline$u$ & Local mean velocity, $x$-direction \\
\hline$u^{\prime}$ & Local RMS fluctuating velocity, $x$-direction \\
\hline$u^{+}$ & Normalized local mean velocity for the law of the wall, $x$-direction \\
\hline$u_{t}$ & Friction velocity \\
\hline$U_{e}$ & Edge velocity; also effective velocity \\
\hline$U_{r e f}$ & Free stream reference velocity \\
\hline$v$ & Local mean velocity, $y$-direction \\
\hline$v^{\prime}$ & Local RMS fluctuating velocity, $y$-direction \\
\hline$w$ & Local mean velocity, $z$-direction \\
\hline$w^{\prime}$ & Local RMS fluctuating velocity, $z$-direction \\
\hline$x$ & Streamwise position, measured downstream of primary trip \\
\hline $\boldsymbol{x}$ & Vector of observer location, $\left(x_{1}, x_{2}, x_{3}\right)$ \\
\hline$X_{1}, X_{2}, X_{3}$ & $\begin{array}{l}\text { Observer position measured from the center of the rotor, streamwise, } \\
\text { spanwise, and normal to wall directions respectively }\end{array}$ \\
\hline$y$ & Spanwise position, measured from the tunnel centerline \\
\hline$z$ & Normal to wall position, measured from the wall \\
\hline
\end{tabular}


Normalized distance away from the wall for the law of the wall

\section{Greek}

$\begin{array}{ll}\gamma & \text { Coherence } \\ \delta & \text { Boundary layer thickness } \\ \delta^{*} & \text { Boundary layer displacement thickness } \\ \kappa & \text { Von Kármán constant } \\ \lambda & \text { Eigenvalue } \\ \nu & \text { Kinematic viscosity } \\ \omega & \text { Angular frequency (radians) } \\ \rho & \text { Density } \\ \Sigma_{n} & \text { Planform area } \\ \tau & \text { Time delay } \\ \tau_{w} & \text { Wall shear } \\ \varphi & \text { Basis function; also, rotor angle } \\ \theta & \text { Boundary layer momentum thickness; also, angle from the } \mathrm{x}_{1} \text { axis } \\ \Omega & \text { Rotor angular velocity }\end{array}$




\section{List of Tables}

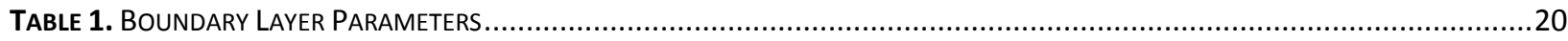

TABLE 2. BLADE ANGLES ALONG THE RADIAL DISTANCE OF EACH BLADE MEASURED FROM THE PLANE OF THE ROTOR .........................48

\section{List of Videos}

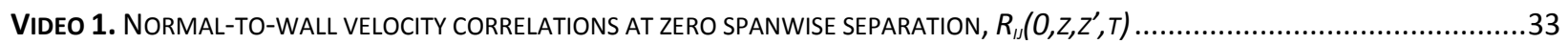

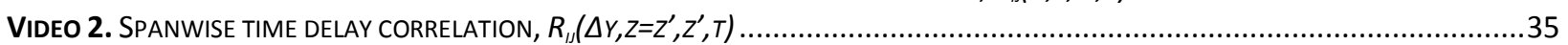

VIDEO 3. STREAMWISE VELOCITY CORRELATION $R_{11}$ SEEN BY THE ROTOR BLADE TIPS AT VARIOUS BLADE SEPARATIONS.......................50

\section{List of Figures}

Figure 2.1. OVerVieW of the Virginia TeCh Stability Wind TUNnel (COURTESy of VT AOE Department).....

FIGURE 2.2. DIAGRAM OF THE VIRGINIA TECH STABILITY WIND TUNNEL WITH FULL ANECHOIC CONFIGURATION INSTALLED (LEFT) AND MODIFIED VERSION WITH FALSE WALL INSTALLED (RIGHT). FIGURE ADAPTED FROM FOREST (2012).

FIGURE 2.3. PLASTIC PLUGS INSTALLED TO FILL INSTRUMENTATION HOLES IN THE LEXAN AND MAINTAIN A HYDRAULICALLY SMOOTH FLOW OVER THE WALL.

FIGURE 2.4. RAILS TO MOUNT THE LEXAN PANELS AND TRAVERSE SYSTEM INSTALLED ON THE FLOOR OF THE TEST SECTION, FIGURE ADAPTED FROM FOREST (2012).

FIGURE 2.5. BACK VIEW OF A TYPICAL FALSE WALL PANEL. FIGURE ADAPTED FROM FOREST (2012) . ...........................................

FIGURE 2.6. TEST SURFACE, MODIFIED CONTRACTION, AND PRIMARY TRIP INSTALLED IN THE TEST SECTION....................................11

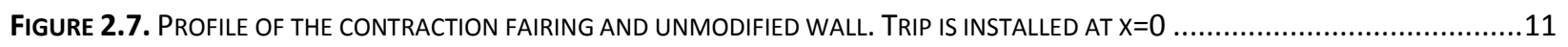

FIGURE 2.8. TOP VIEW SCHEMATIC OF THE TEST SECTION WITH FALSE WALL INSTALLED. ...................................................12

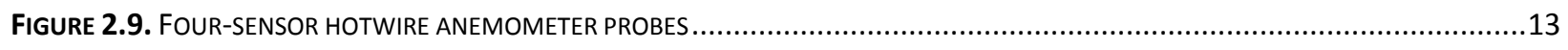

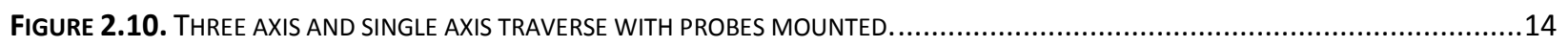

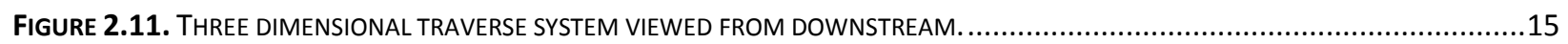

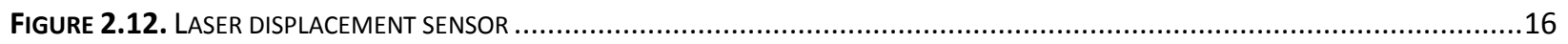

FIGURE 2.13. QUADWIRE PROBES AT THEIR MINIMAL Y SEPARATION OF 4 MM (FROM CENTER TO CENTER OF MEASUREMENT VOLUMES).17

FIGURE 2.14. ONE OF 31 TWO-POINT GRIDS USED IN MEASURING THE FOUR-DIMENSIONAL TWO-POINT SPACE TIME CORRELATION, SUPERIMPOSED ON AN ESTIMATE OF THE ZERO TIME DELAY CORRELATION BASED ON MOSER ET AL.'S (1999) DNS CHANNEL FLOW

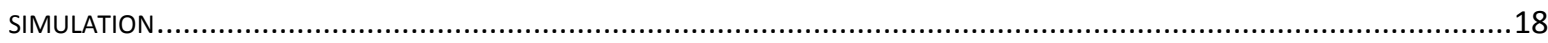

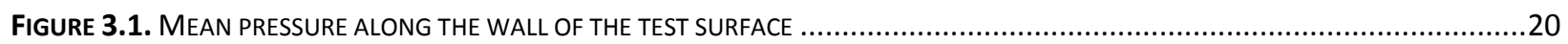

FIGURE 3.2. MEAN VELOCITY PROFILES. BLACK, BLUE, AND RED REPRESENT 20, 30, AND $55 \mathrm{M} / \mathrm{S}$ RESPECTIVELY.............................21

FIGURE 3.3. MEAN VELOCITY COMPARISON BETWEEN SINGLE-POINT QUADWIRE, TWO-POINT QUADWIRE, AND CONCURRENT STUDY WITH

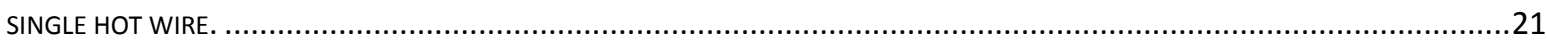

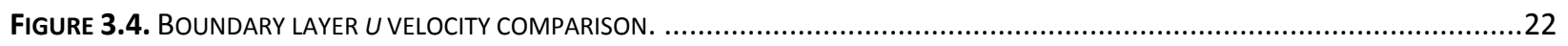

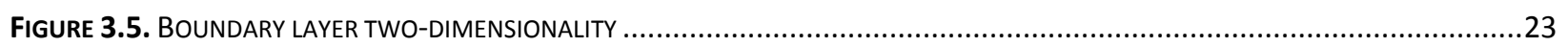

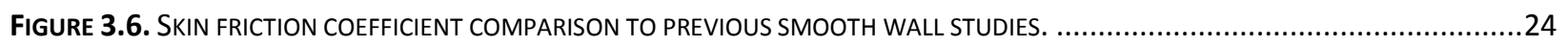

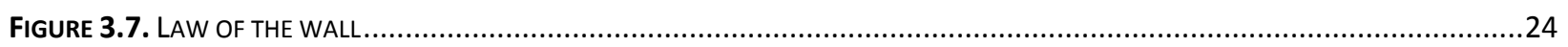

FIGURE 3.8. (A) TURBULENT NORMAL STRESSES AND NON-ZERO REYNOLDS STRESS PROFILES NORMALIZED ON UE ${ }_{\mathrm{E}}^{2}$. BLACK REPRESENTS $20 \mathrm{M} / \mathrm{s}$, BLUE $30 \mathrm{M} / \mathrm{s}$, AND RED 55M/s. (B) $U^{\prime 2}$ NORMAL STRESS NORMALIZED ON INNER REGION VARIABLES ..........................26

FIGURE 3.9. AUTOSPECTRA OF VELOCITY FLUCTUATIONS MEASURED AT VARIOUS POSITIONS WITHIN THE BOUNDARY LAYER. SPECTRAL LEVELS AND FREQUENCIES ARE MULTIPLIED BY 0.1, 0.01 AND 0.001 FOR $Z / \Delta=24 \%, 54 \%$ AND 101\%, RESPECTIVELY.............27

FIGURE 3.10. REYNOLDS STRESS PROFILES OF THE MOVING AND FIXED PROBE BEFORE APPLYING A TRANSFORMATION TO THE MOVING

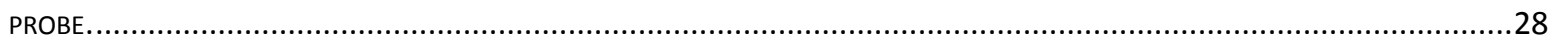

FIGURE 3.11. REYNOLDS STRESS PROFILES AFTER APPLYING A TRANSFORMATION TO THE MOVING PROBE....................................29

Figure 3.12. Comparison of Reynolds Stress Profiles from Non-Processed DATA ANd Zero-Time Delay $R_{I J}\left(Z=Z^{\prime}\right)$. BLACK

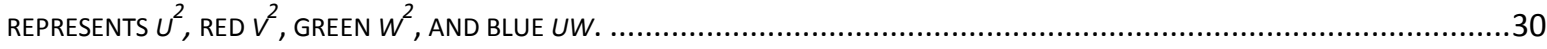

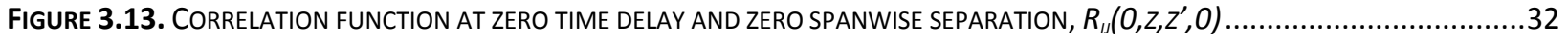


Figure 3.14. COMPARISON BETWEEN THE CORRELATION FUNCTION OF MOSER (TOP) AND THE PRESENT STUDY (BOTTOM) AT A FIXED PROBE LOCATION OF $Z / \triangle=20 \%$.

FIgURE 3.15. COMPARISON BETWEEN THE CORRELATION FUNCTION OF MOSER (TOP) AND THE PRESENT STUDY (BOTTOM) AT A FIXED

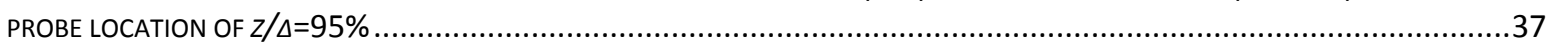

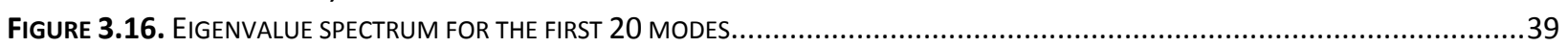

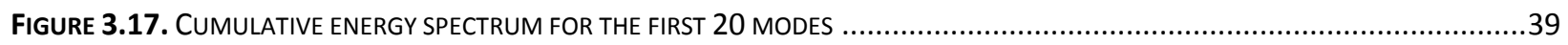

FIGURE 3.18. MOdAL PROFILES OF THE FIRST FOUR MODES DETERMINED FROM PROPER ORTHOGONAL DECOMPOSITION (POD).........40

FIGURE 3.19. COMPACT EDDY STRUCTURES OF THE FIRST FOUR MODES WITH EIGENVALUE NORMALIZATION ....................................42

FIGURE 3.20. COMPACT EDDY STRUCTURES FOR MODE 1 (TOP) AND MODE 2 (BOTTOM) AT VARIOUS NORMAL TO WALL LOCATIONS ......43

FIGURE 3.21. COMPACT EDDY STRUCTURES FOR MODE 3 (TOP) AND MODE 4 (BOTTOM) AT VARIOUS NORMAL TO WALL LOCATIONS ......44

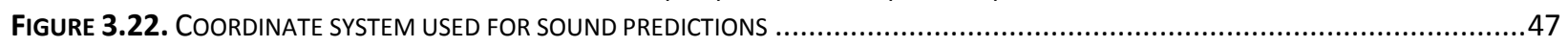

FIGURE 3.23. SCHEMATIC OF THE ROTOR WITH PROFILES OF THE BLADES AT THE TIP AND ROOT ...............................................48

FIGURE 3.24. SPANWISE COHERENCE BETWEEN VELOCITY FLUCTUATIONS AT THE BLADE TIP AND VARIOUS RADIAL LOCATIONS ON THE SAME BLADE. $(R N=1, P=0)$

FigURE 3.25. BLADE TO BLADE COHERENCE BETWEEN VELOCITY FLUCTUATIONS SEEN AT THE BLADE TIP FOR DIFFERENT BLADE NUMBER

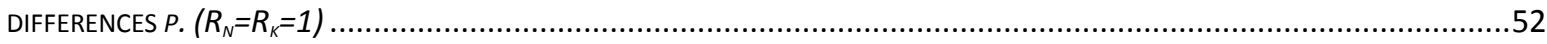

FIGURE 3.26. BLADE TO BLADE COHERENCE BETWEEN VELOCITY FLUCTUATIONS SEEN AT THE BLADE TIP AND AT THE $95 \%$ RADIAL

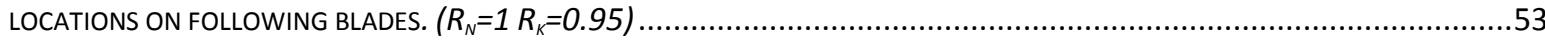

FIGURE 3.27. BLADE TO BLADE COHERENCE BETWEEN VELOCITY FLUCTUATIONS SEEN AT THE BLADE TIP AND AT THE $90 \%$ RADIAL

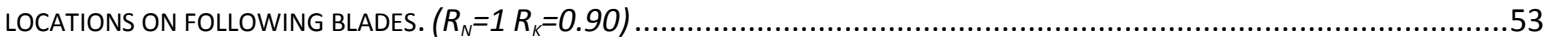

Figure 3.28. BLADE TO BLADE COHERENCE BETWEEN VELOCITY FLUCTUATIONS SEEN AT THE BLADE TIP AND AT THE 85\% RADIAL

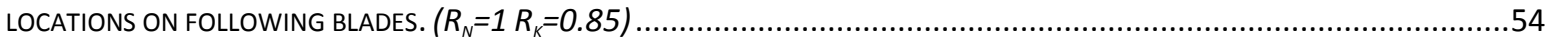

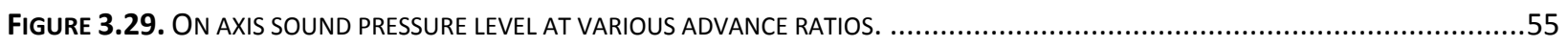

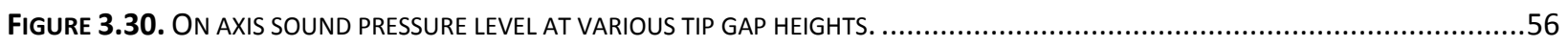

FIGURE 3.31. ON AXIS SOUND PRESSURE LEVEL AT FREE STREAM VELOCITIES OF 20 AND 30M/s.............................................56

FIgURE 3.32. SOUND PRESSURE LEVELS AT VARIOUS ANGLES ABOUT THE $X_{2}$ AXIS ( $\left.\Phi=90\right)$. THE DASHED BLACK LINE REPRESENTS THE FIRST

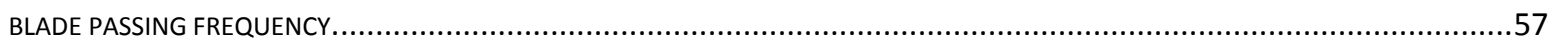

FIGURE 3.33. FAR-FIELD SOUND LEVELS ABOUT THE $X_{2}$ AXIS WITHOUT THE EFFECT OF THE WALL. CONTOUR LEVELS ARE $S_{P P}$ [DB] (REL.

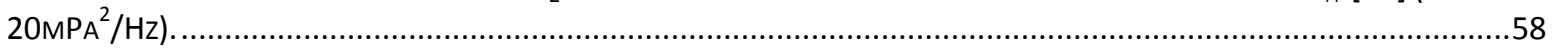

Figure 3.34. FAR-FIELD SOUND LEVELS ABOUT THE $X_{3}$ AXIS WITHOUT THE EFFECT OF THE WALL. CONTOUR LEVELS ARE $S_{P P}$ [DB] (REL.

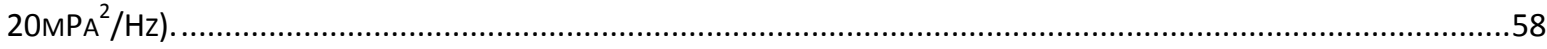

FIGURE 3.35. EFFECT OF THE WALL ON THE FAR-FIELD SOUND AT AN OBSERVER LOCATED 5M DOWNSTREAM ON THE AXIS OF THE ROTOR

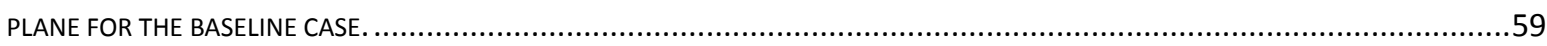

Figure 3.36. FAR-FIELD SOUND LEVELS ABOUT THE $X_{2}$ AXIS WITH THE EFFECT OF THE WALL. CONTOUR LEVELS ARE $S_{P P}$ [DB] (REL. $\left.20 \mathrm{MPA}^{2} / \mathrm{Hz}\right)$.

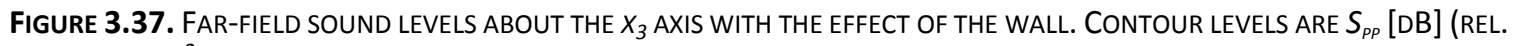
$\left.20 \mathrm{MPA}^{2} / \mathrm{Hz}\right)$. 


\section{CHAPTER 1 INTRODUCTION}

\section{$1.1 \quad$ Motivation}

The interaction between a rotating system and a turbulent boundary layer has been the focus of many studies since it is a significant source of noise and vibration in aircraft engines. However, the scenario of a rotor ingesting a plane boundary layer with thickness comparable to the rotor radius has not been explicitly studied. This problem involves the effects of mean flow shear which complicates the traditionally used rapid distortion theory. The shear flow turbulence is distorted as it is ingested into the rotor which results in an unknown response by the rotor blades. This thesis thus presents the efforts to achieve an accurate description of the interaction between a rotor and a turbulent boundary layer and the noise that this interaction generates utilizing the Virginia Tech Stability Wind Tunnel facility.

\section{$1.2 \quad$ Literature Review}

A rotor interacting with a nonuniform turbulent inflow generates unsteady forces on the blade surfaces which can be a source of acoustic radiation and mechanical vibration. The reduction of such noise and vibration is highly desirable to reduce an aircraft's acoustic signature and ensure proper operation of the engines drive system. A prime example of the occurrence of this interaction is the embedded aircraft engine. The Silent Aircraft Initiative is a recent collaboration between academia, industry, and government agencies to reduce the noise generated by an aircraft, including the effects of embedded engines (Hileman et al. 2007). The final design of this effort involves embedding multiple engines at the trailing edge of a Blended-Wing-Body (BWB) each of which ingest the suction side boundary layer. Plas (2006) shows that, for this design at typical flight conditions, the ingestion of a boundary layer can provide a significant decrease in fuel burn. However, as discussed by de la Rosa (2007), this new concept introduces difficulties in predicting the noise generated since the engines are now ingesting boundary layer turbulence.

Sevik (1970) was one of the earliest experimental studies that investigated turbulence ingestion into a rotor. In this study a 10 bladed, 8 inch diameter propeller was mounted in a water tunnel and subjected to a turbulent inflow generated by turbulence grids. The ingested turbulence was assumed to be isotropic and homogeneous. Experimental measurements of the unsteady thrust and theoretical calculations exhibit the same overall trend with the exception of "haystacks" in the measured data which occur at multiples of the blade passing frequency. These haystacks occur when multiple blades cut through the same turbulent structure meaning fluctuating loads are correlated between blades, something Sevik did not take into consideration. This result prompted future studies to incorporate the possibility that fluctuating loads may be correlated between blades (Blake, 1986, Martinez, 1997, Glegg and Walker, 1998, Joseph and Parry, 2001, Attassi and Logue, 2009). Blade to blade correlation is governed by two main factors: the rotor's advance ratio and the distortion of turbulence as it is drawn into the rotor. A small advance ratio means the rotor is spinning at high speeds relative to the incoming flow and as a result adjacent blades are more likely to cut the same structure. A thrusting rotor causes distortion of turbulence by accelerating the flow as it approaches the fan face consequently stretching the incoming turbulence in the streamwise direction. This 
stretching also increases the possibility that multiple blades will cut through the same structure.

The study of Hanson (1974) demonstrates an extreme case of turbulence ingestion. Hanson examined the noise generated by the ingestion of atmospheric turbulence into a stationary shrouded rotor. Measurements of the turbulent velocities were performed at the fan inlet using hot-wire anemometry to provide an aerodynamic description of the inlet turbulence and measurements of the blade pressure response performed using pressure transducers embedded in the fan blades. He found the streamwise scale of the turbulence to be over 100 diameters while the transverse scale was only a fraction of a diameter. This equated to a 400:1 streamwise to cross stream aspect ratio which caused narrow peaks in the spectral data resulting from multiple blades cutting the same structure. Majumdar and Peake (1998) developed a model to predict the noise generated from the interaction of ingested atmospheric turbulence with a rotating fan using rapid distortion theory and assuming isotropic turbulence. They determined that the contraction of the inflow is much stronger under static conditions than at typical flight conditions but the effect of turbulence distortion during normal flight is still relevant.

Many studies on the ingestion of less distorted turbulence have been performed in the anechoic wind tunnel at the University of Notre Dame. Sharpf and Mueller (1995) studied the noise radiation from a four bladed, 10 inch diameter propeller operating in air at $3000 \mathrm{rpm}$ and free stream flow of $33 \mathrm{ft} / \mathrm{s}$ using hotwire anemometry and microphones. Three different turbulence grids located upstream of the rotor were used to generate turbulence levels from 0.2 to $5.5 \%$. Blade to blade correlation was only observed when the turbulence grids were removed and the larger eddies generated by the facility interacted with the rotor. The radiated noise level was found to increase by $2 \mathrm{~dB}$ for every $1 \%$ increase in the turbulence levels. In the same facility Wojno et al. $(2002,2002 \mathrm{a})$ used similar turbulence generating grids to measure the far-field noise generated by the 10bladed Sevik rotor. The cross spectrum between the fluctuating velocity components of two hotwire probes was measured over a detailed cross section $11 \mathrm{~cm}$ upstream of the rotor then converted to the rotating reference frame by assuming Taylor's hypothesis. Theoretical predictions were made in the spectral domain using strip theory and the summation gain approach of Blake (1986), which account for blade to blade correlation. Predictions match well with measurements especially for broadband levels over the frequency range of 1 to $3 \mathrm{BPF}$. However, the narrowband response around the blade passing frequency suggests blade to blade correlation was underestimated. Minniti et al. (2001, 2001a) developed techniques to infer inflow characteristics based on the unsteady pressure seen on the blades of a 4 bladed propeller ingesting turbulence. The study considered both large-scale turbulence and grid generated turbulence with different characteristic eddy sizes. Unsteady pressure measurements were performed using a total of nine PVDF unsteady pressure transducers mounted at different locations on each blade. The sensors were arranged in such a way that allowed the blade to blade correlation and the spanwise correlation of unsteady lift to be measured. Hotwire measurements were also performed in the plane of the rotor by mounting a probe on a blade just forward of its leading edge. Unsteady pressure, inflow, and far-field measurements all agreed well with predictions. However, the blade spacing on this four bladed rotor is relatively large compared to the measured turbulent scales. Lynch et al. (2005, 2005a, 2005b) studied the aeroacoustic response of an idealized stator, represented by a flat-plate airfoil, 
downstream of a rotor. Hotwire measurements were made at the location of the airfoil for multiple propellers with blade counts of 4,10, and 20, respectively, to determine the effects of blade spacing. Based on the rapid distortion theory predictions of Graham (1998), a technique was developed to predict the levels of turbulence suppression downstream of the rotor. After separating the effects of the ingested turbulence from the propeller wake, predictions and measurements matched well.

Most studies of boundary layers being drawn into rotors have focused on axisymmetric casing and hub flows; not the ingestion of thick plane boundary layer flows such as those present in embedded engines. Traditional airplane applications involve thin boundary layers and thus research tends to focus in that area. A well-known study was performed Ganz et al. (1998) which analyzed the fan tip/boundary layer interaction of the Boeing 18-inch fan. This model was a high-bypass-ratio fan with an extended inlet duct equipped with a perforated wall suction system to allow for the removal of the boundary layer. Comparisons between the measurements with and without the $1 \mathrm{~cm}$ thick inlet boundary layer demonstrate that the interaction between the blade tips and the boundary layer is a significant source of noise. Measurements also indicate the effects of haystacking which suggests the presence of blade to blade correlation. Glegg and Walker (1998) later modeled experiment of Ganz using a rectilinear approximation of the ducted fan. This study found that by using an anisotropic model with axial length scales ten times larger than transverse scales, a good representation of the spectral humps is achieved. However, this ratio is much larger than the measured ratio of Ganz. The Ganz experiment was also modeled by Joseph and Parry (2001) using an approach previously developed by Gavin and Lauchle (2000). Similar results were found in this study in that the measured ratio of length scales is too small to account for the haystacking effect.

More recently, Stephens and Morris (2009) performed a study focusing on the interaction between the blade tips of the Sevik rotor and a boundary layer formed from a circular duct surrounding the rotor. The rotor was mounted in a $206 \mathrm{~mm}$ diameter duct leaving a gap between the duct and blades of $1.4 \mathrm{~mm}$. To vary the thickness of the boundary layer that was interacting with the blades, the rotor was positioned at different streamwise locations. In order to determine the rotor's radiated self-noise the rotor was placed at a location $2.54 \mathrm{~cm}$ downstream from the inlet where the flow was essentially steady and axisymmetric. With the rotor near zero thrust, the noise produced from the interaction of the boundary layer with the blade tips was studied with the rotor at an axial location of 1.125 rotor diameters downstream from the inlet where the boundary layer was thickest and measured to be $11 \mathrm{~mm}$. Measurements of the two-point cross correlation of axial velocity were made in the boundary layer. This correlation was then extrapolated into the full 4-Dimensional correlation function by assuming an exponential decay in the spatial separation distance that is proportional to the magnitude of the autocorrelation. From this correlation function Stephens and Morris determined the unsteady loading on the blades and used this to determine the radiated sound. Noise predictions were found to agree well with experimental measurements.

Other studies have focused on hub boundary layer interaction. Moiseev (1978) studied the effects of different turbulent inflows generated by a rotor hub interacting with a 10 and 17 bladed rotor. Measurements were performed for the natural hub boundary layer condition, a tripped boundary layer on the hub, and a fully developed hub boundary layer. The inlet was sufficiently long to ensure that the turbulence from the hub and casing 
fully enclosed the rotor cross section. Turbulence properties were also changed by adding a turbulence grid at the inlet. Aerodynamic measurements include single-point hotwire measurements at the inlet to determine mean velocities and turbulence intensities, and two-point hotwire measurements to determine the cross-correlation between the circumferential components of turbulent fluctuations. Far-field sound measurements were performed 4.35 duct diameters upstream of the inlet duct opening in the anechoic chamber. The turbulence grid greatly reduced the integral scales but axial scales were still significantly larger than circumferential scales. The sound levels were found to vary as the square of turbulent intensities and inversely to the ratio of axial length scale to blade spacing.

Muench (2001) studied the effect of wake deficits generated by a stator row just upstream of a 6 bladed rotor which causes unsteady forces on the blades and therefore also radiated noise. This configuration was mounted at the end of an axisymmetric body. Detailed measurements were performed over a cross section of the flow between the stator row and plane of the propeller using hotwire probes. Muench also used 15 unsteady pressure sensors embedded in the blades of the rotor to measure the spanwise, chordwise, and blade to blade unsteady pressure correlations. This study was then continued by Huyer and Snarski (2003) who performed additional measurements in the rotating frame. From these measurements the axial, radial, and circumferential velocity components were determined which allowed the effects of the turbulent hull boundary layer to be separated from the induced velocity of the stator wakes and the induced velocity from the rotor. The inflow was found to have a region close to the hub of increased turbulence levels suggesting that the axisymmetric body was the main contributor to the distortion of turbulence as opposed to the ingestion from the rotor. Huyer and Beal (2007) expanded on this work by using the previously measured inflow to predict the far-field radiated noise. The model used for noise predictions was based on the alteration of the velocity inflow to match previous hotwire measurements. Far-field noise simulations were found to accurately predict the sound field for the first two blade passing frequencies but, at higher frequencies only the broadband component was accurate.

The majority of studies previously discussed have used rapid distortion theory as a basis for their calculations. However, a thick turbulent boundary layer generally has a mean flow shear which cannot be ignored. This largely affects rapid distortion theory meaning the effects of mean flow shear may have influenced the studies on hub and casing boundary layer flows above.

Recently a study was performed by Catlett et al. (2012) with many similarities to the present study, which was by design. The focus of this study was to investigate the radiated sound generated by a propeller ingesting a sheared, spatially inhomogeneous, turbulent flow. A 7 bladed 11.52" diameter rotor was mounted at the trailing edge of an airfoil of $10 \mathrm{ft}$ chord to create a sheared turbulent velocity field at the rotor inlet. Measurements were performed using two hotwire probes traversed through a 3 dimensional measurement volume to determine the two-point statistics of the flow field in the plane of the rotor as well as microphones mounted in the far-field downstream of the rotor to determine the radiated sound. Theoretical predictions were performed by applying a coordinate system transformation to the acoustic compact dipole model to incorporate the effects of anisotropy in the flow. The predicted sound generated based on the unsteady velocities measured in the flow agree well with corresponding acoustic measurements. 


\section{$1.3 \quad$ Objectives}

The purpose of this study is to analyze the interaction between a rotor and the thick turbulent boundary layer in which it is immersed by removing unwanted complexities that mask the underlying physics. The objectives of the study are as follows:

- Generation in a controlled laboratory environment of a fully developed very high Reynolds number turbulent boundary layer that is thick enough to immerse an idealized rotor configuration.

- Document the two-point space time correlation structure of the undisturbed boundary layer.

- Determine the correlation seen by a theoretical rotor as it cuts through the boundary layer.

- Use the theory developed by Glegg, which accounts for the interaction of each blade with the anisotropy of the turbulence, to predict the noise generated from the rotor.

- Examine the effect of various operating conditions on the noise predictions. 


\section{CHAPTER 2 APPARATUS AND INSTRUMENTATION}

\subsection{Virginia Tech Stability Wind Tunnel}

All data presented were taken in the Virginia Tech Stability Wind Tunnel, as it is particularly well suited to velocity and sound measurements at high Reynolds number. The Stability Tunnel is a continuous, single return, subsonic wind tunnel capable of achieving speeds up to $80 \mathrm{~m} / \mathrm{s}$ in the test section. The facility features interchangeable test sections allowing the tunnel to run in an anechoic configuration that has been shown to be acoustically quiet without disturbing the aerodynamics of the flow (Devenport, et al. 2010). Turbulence levels at the inlet of the test section have been measured to be $0.021 \%$ and $0.031 \%$ at $21 \mathrm{~m} / \mathrm{s}$ and $57 \mathrm{~m} / \mathrm{s}$ respectively.

Power to the tunnel is generated by a $0.45 \mathrm{MW}$ variable speed DC motor with the capability of drawing up to 900 Amps. The motor is controlled by a custom built Emerson VIP ES-6000 SCR Drive which is used to limit any cyclic unsteadiness introduced by the motor. The motor is directly connected to the fan which consists of 8 Clark-Y airfoil section blades and measures 4.27 meters in diameter. Foam has been added to reduce the clearance between the fan and wall thus reducing the noise generated by the blade tips by 5 to $6 \mathrm{~dB}$ across the entire frequency range (Remillieux, et al. 2008). A schematic of the wind tunnel can be seen in Figure 2.1.

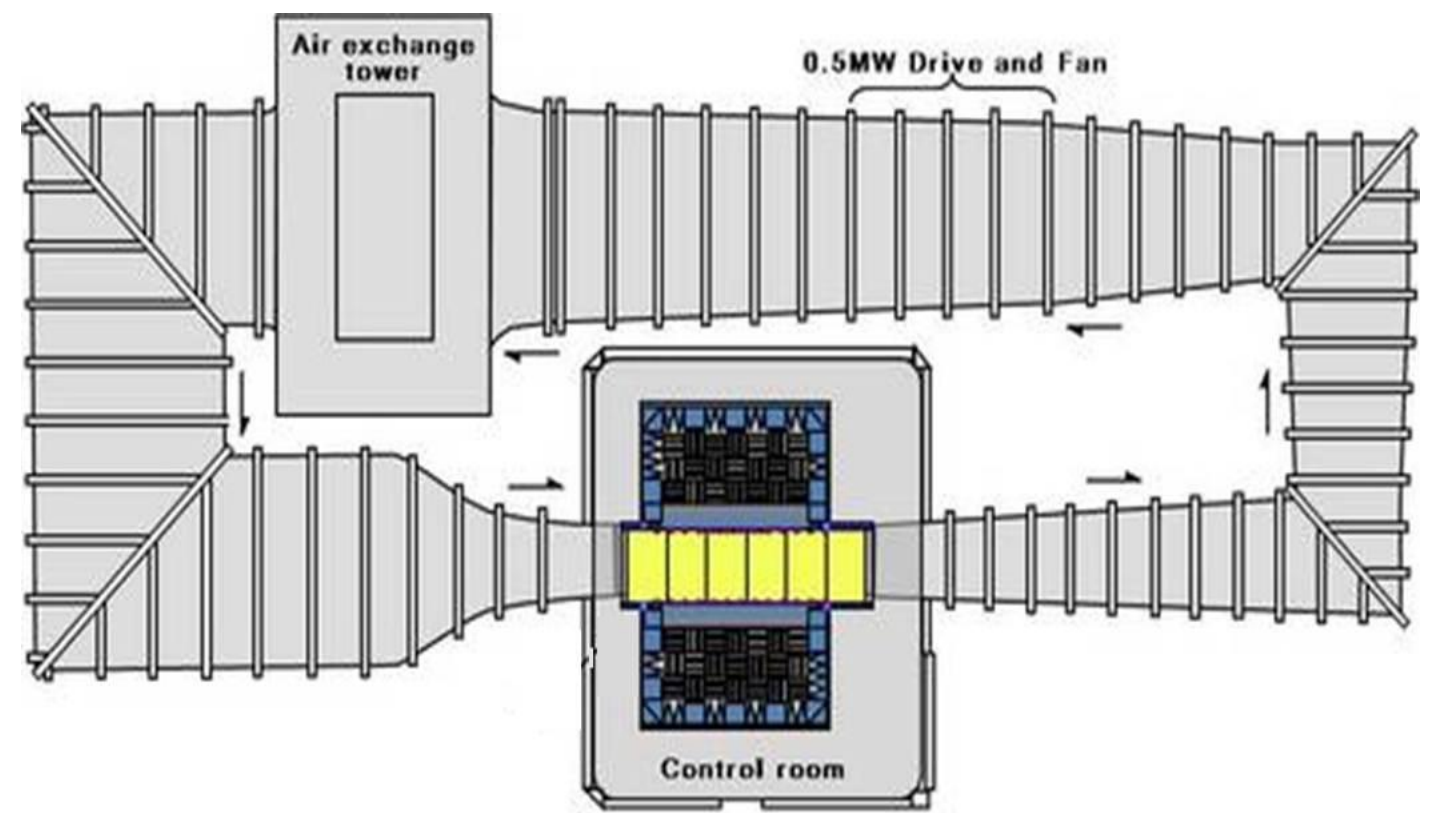

Figure 2.1. Overview of the Virginia Tech Stability Wind Tunnel (Courtesy of VT AOE Department)

The tunnel forms a closed loop, but has an open to atmosphere air exchange tower to allow for temperature stabilization. The air exchange tower is located directly downstream of the fan and motor assembly. Following the exchange tower the flow enters a $5.5 \mathrm{~m}$ square settling chamber which contains 7 turbulence reduction screens with a $60 \%$ open air ratio. The walls of the settling chamber are overlaid with $50 \mathrm{~mm}$ thick urethane foam for acoustic treatment. After the turbulence screens the flow is directed through a 9:1 
contraction nozzle which further reduces turbulence levels and accelerates the flow into the test section.

The anechoic configuration shown in Figure 2.2 consists of an acoustic test section flanked by two large acoustic chambers. The test section has a 1.83 meter square cross section and is 7.3 meters long. The floor and ceiling of the test section are covered in perforated aluminum panels which are draped in stretched Kevlar cloth and have been shown to create a quiet, smooth, acoustically transparent flow surface. Above and below the test section on the other side of these perforated panels exists 0.457 meter high foam wedges arranged in alternating directions. The two acoustic chambers are separated from the test section by stretched Kevlar windows each 6 meters long and 1.83 meters high. Sound generated in the test section exits through these Kevlar windows into the acoustic chambers. Acoustically this configuration simulates a half-open jet and eliminates the need for a jet catcher. Both chambers have a streamwise length of 5.6 meters, width of 2.8 meters, and height of 4.2 meters and are lined with 0.61 meter high acoustic wedges. In this study the Kevlar wall on the starboard side of the test section was replaced with a custom built false wall and the starboard chamber was used to store the measurement instrumentation. The false wall reduced the test section width to 1.73 meters and is further described in section 2.2.1.
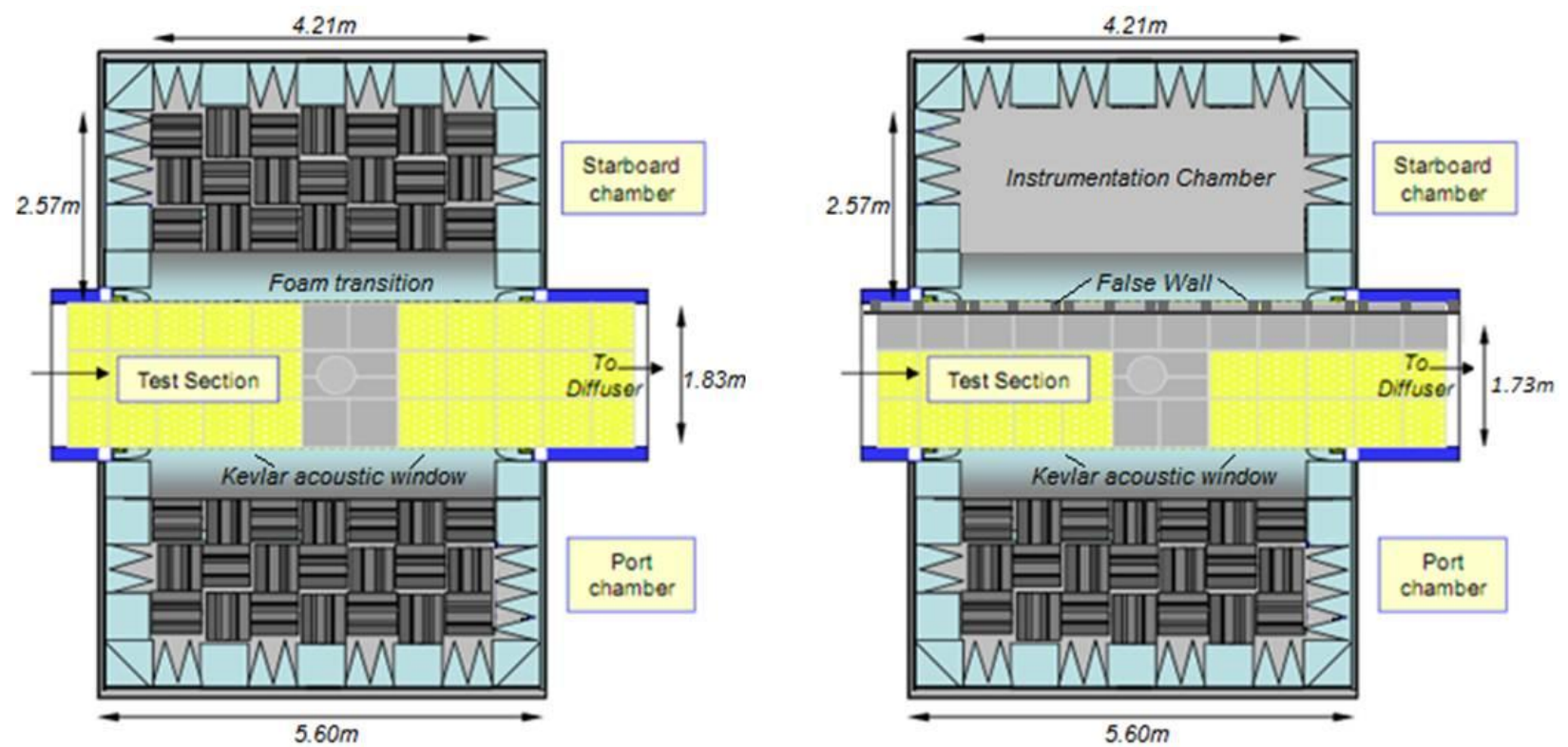

Figure 2.2. Diagram of the Virginia Tech Stability Wind Tunnel with full anechoic configuration installed (left) and modified version with false wall installed (right). Figure adapted from Forest (2012).

Following the test section, the flow passes into a 3 degree diffuser containing 16 vortex generators equally spaced around the walls, floor, and ceiling. To prevent high frequency noise from travelling to the test section from the fan, $50 \mathrm{~mm}$ thick melamine foam lines walls and ceiling of the diffuser. After the diffuser the flow travels passed two sets of turning vanes and is redirected back to the fan.

\section{$2.2 \quad$ Testing Surface and Instrumentation}




\subsubsection{False Wall}

The anechoic test section has been modified in order to install a false wall consisting of 6 Lexan panels in place of the starboard side Kevlar wall. The purpose of the false wall is to create a test surface on which to grow large, high Reynolds number turbulent boundary layer flows.

The false wall was custom built for previous research efforts by Forest (2012). The wall consists of six $13 \mathrm{~mm}$ thick Lexan panels each $1.78 \times 1.22$ meters. The back of each panel was bonded to an $80 / 20$ aluminum frame providing a solid structure and the availability for instrument connections. Instrumentation slots were milled out of the panels for collaborative studies but these were sealed to maintain a hydraulically smooth flow over the test surface. For the larger slots $\left(>1 \mathrm{~cm}^{2}\right)$ plastic plugs were created by an Objet Alaris 30 3D printer and designed to sit flush with the test surface as shown in Figure 2.3. Smaller instrumentation slots were covered with packing tape less than $0.076 \mathrm{~mm}$ thick.

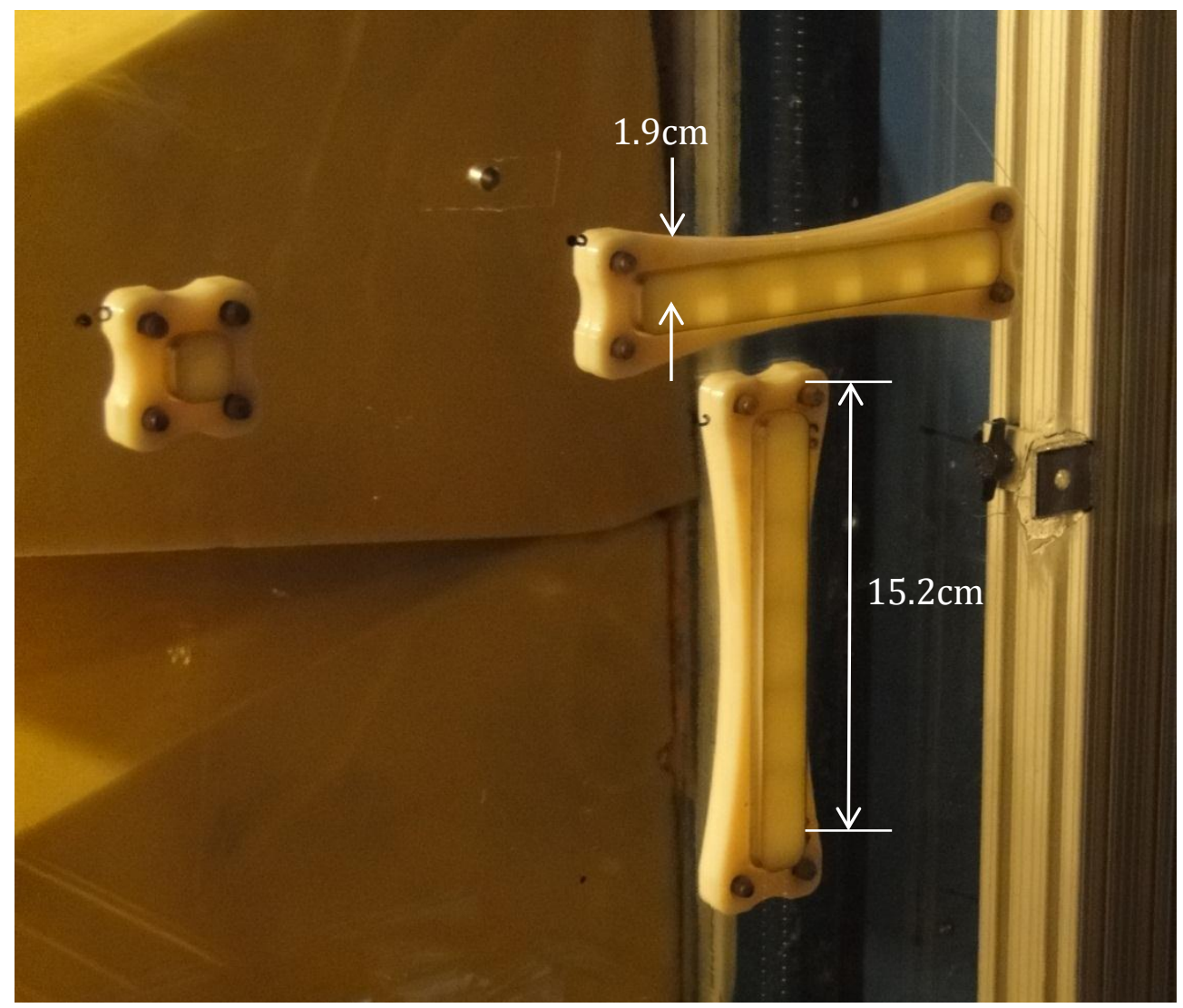

Figure 2.3. Plastic plugs installed to fill instrumentation holes in the Lexan and maintain a hydraulically smooth flow over the wall.

In order to install the false wall in the test section, 12 of the perforated Kevlar panels on the ceiling and another 12 on the floor closest to the starboard chamber were removed and replaced by equally sized aluminum plates. These aluminum plates permitted a rail system, shown in Figure 2.4, to be installed thus allowing individual positioning of each Lexan panel. Each Lexan panel was connected to the 80/20 rails by 8 right angle brackets, as shown in Figure 2.5. These brackets allowed each panel to be 
maneuvered in both the normal-to-wall and streamwise directions to create a zero pressure gradient flow along the test section.

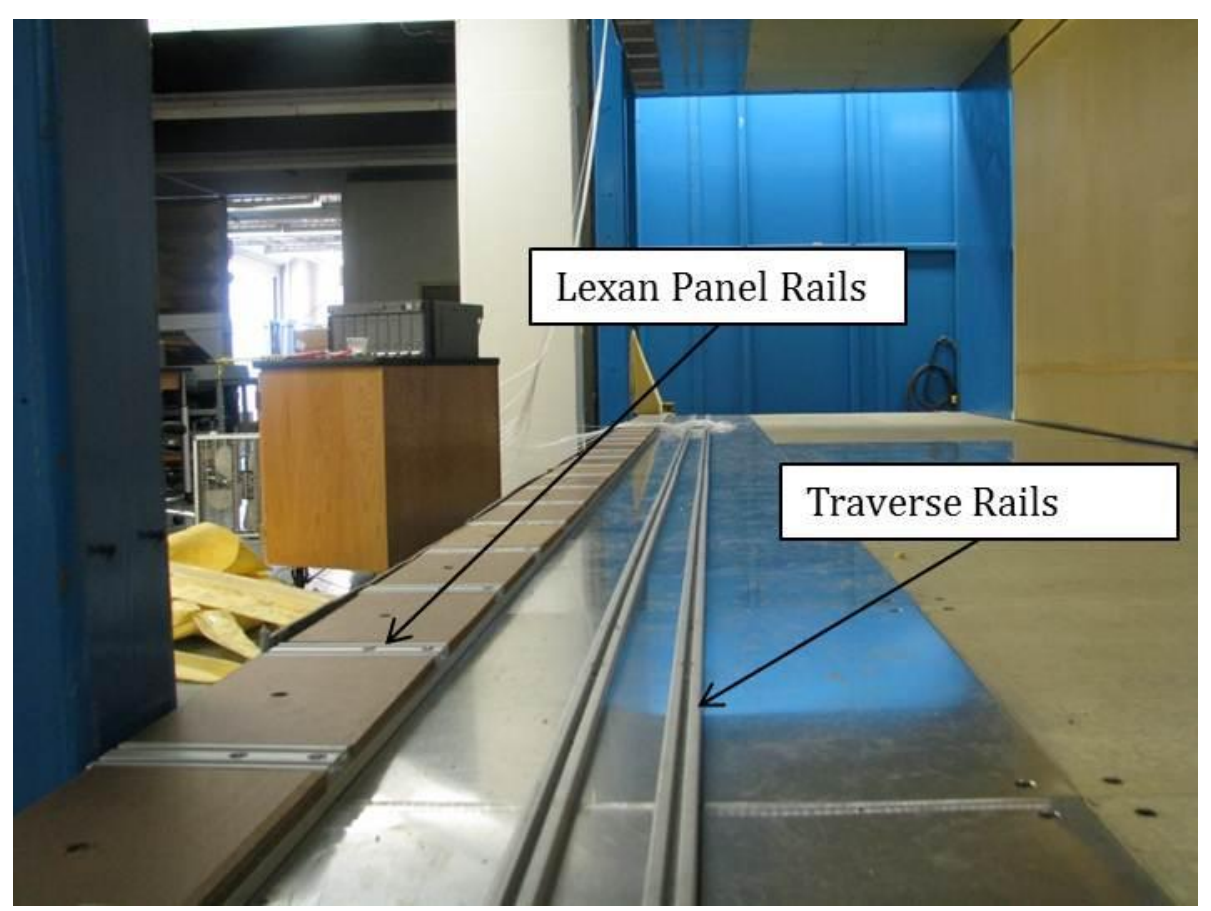

Figure 2.4. Rails to mount the Lexan panels and traverse system installed on the floor of the test section, Figure adapted from Forest (2012). 


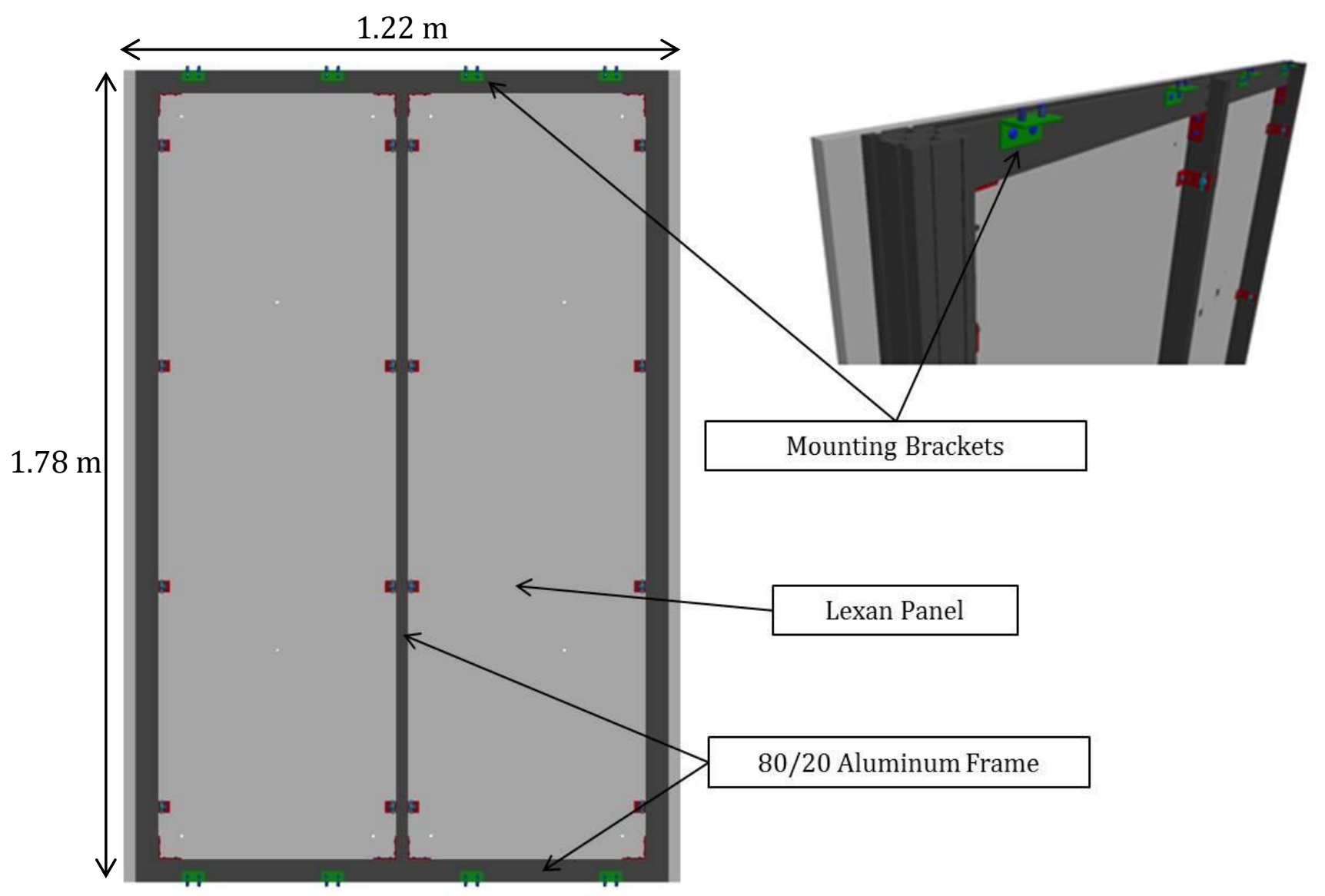

Figure 2.5. Back view of a typical false wall panel. Figure adapted from Forest (2012).

Fully installed, the Lexan panels line up to create an unobstructed 1.78 x 7.22 meter long testing surface. As previously mentioned each panel could be individually adjusted in two dimensions. Each panel was positioned within $6 \mathrm{~mm}$ in the streamwise direction and $0.25 \mathrm{~mm}$ in the normal-to-wall direction to each of its adjacent panels. The gaps between adjacent panels were covered using $0.076 \mathrm{~mm}$ thick packing tape to reduce the effect of any step still present.

Since the false wall reduces the width of the test section a significant step of 0.12 meters occurs at the leading edge of the wall. To overcome this unwanted step a curved fairing was designed to redirect the flow in the contraction of the tunnel over the false wall test surface. A profile at the centerline of the curved fairing can be seen in Figure 2.7 where the coordinate system is described in Section 2.2.2. The red line in Figure 2.7 is a profile of the wall without the fairing installed which is identical to the other three walls of the wind tunnel contraction. The fairing consisted of a wooden structure covered in ABS plastic that sat flush with the leading edge of the false wall and extended 2.4 meters into the tunnel contraction when installed. The contraction fairing was also built to allow trips to be installed at two streamwise locations. The primary location was set at 1.2 meters upstream of the leading edge of the false wall and the secondary located 2.1 meters upstream. This allowed different trip configurations to be installed and thus various turbulent boundary layers to be created. This study required two trip configurations. The first of the configurations used a single $9.5 \mathrm{~mm}$ right angle aluminum trip installed at the primary trip 
location. The second utilized both trip locations and consisted of two $19 \mathrm{~mm}$ right angle aluminum trips. The curved fairing with installed trip can be seen in Figure 2.6.

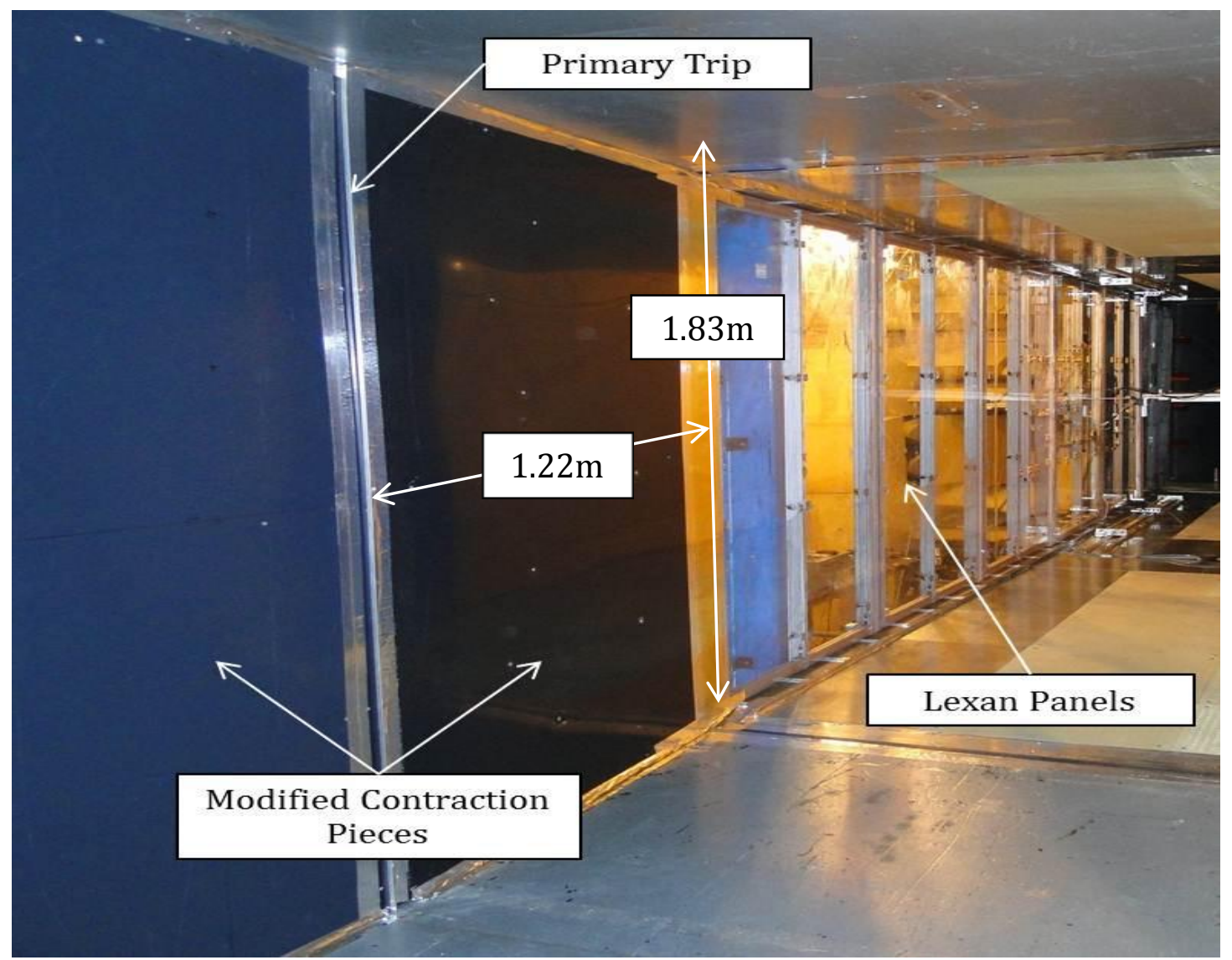

Figure 2.6. Test surface, modified contraction, and primary trip installed in the test section.

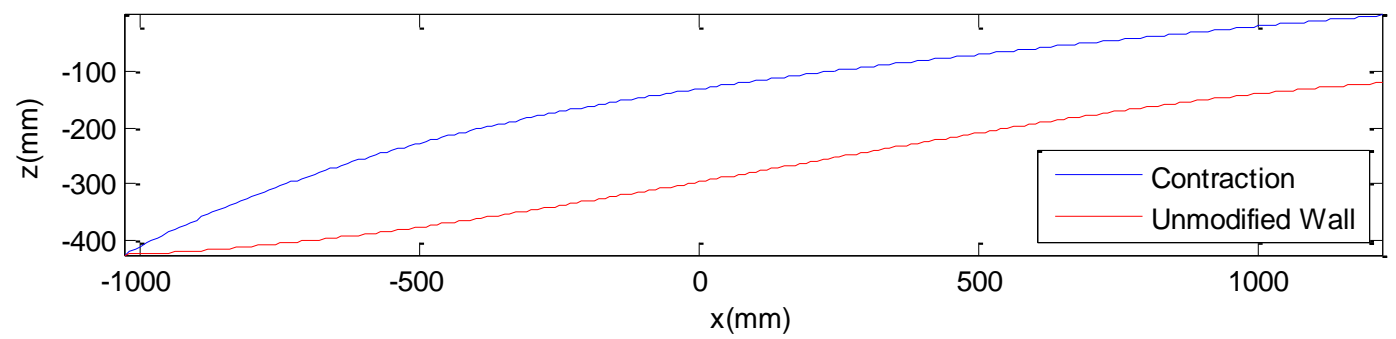

Figure 2.7. Profile of the contraction fairing and unmodified wall. Trip is installed at $\mathrm{x}=0$

\subsubsection{Coordinate System}

The origin used for all measurement locations in the $x$-direction was defined as the primary trip with positive $x$ oriented downstream. All measurements were performed at an $x$ location of 7.72 meters as shown in Figure 2.8. The $y$ axis origin was defined as the midheight of the tunnel with positive oriented upwards. To obtain a right-handed coordinate 
system the $z$ axis origin was defined as perpendicular to the plane of the false wall with positive oriented away from the wall.

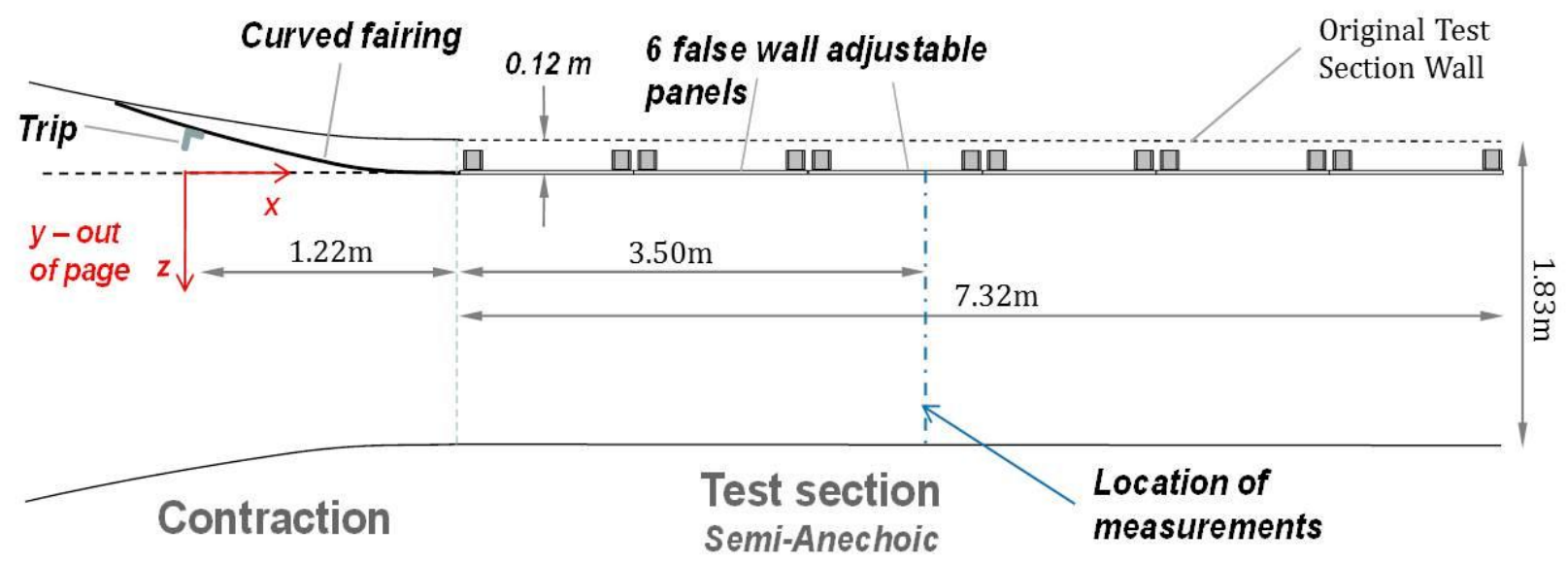

Figure 2.8. Top view schematic of the test section with false wall installed.

\subsubsection{Reference Instrumentation}

Mean wall pressure measurements were made using twenty four $1 / 2 \mathrm{~mm}$ pressure taps located on the floor and ceiling of the test section. The ports were spaced approximately 61 centimeters apart in the $x$-direction spanning the test section between a distance of 1.65 and 8.15 meters from the trip. Mean pressure data was measured using an Esterline NetScanner Model 98RK pressure scanner. This data was then used to adjust the false wall in order to achieve a near zero pressure gradient as discussed in Section 3.2. Along with the mean pressure in the test section this pressure scanner also constantly recorded the ambient pressure in the control room which was used as a reference pressure. A Pitot static tube located at $(x, y, z)=(4.43 \mathrm{~m},-.59 \mathrm{~m}, 1.25 \mathrm{~m})$ was used to measure the free stream static and stagnation pressures and velocity which most measurements are normalized on. Ambient temperature was critical in determining when velocity calibrations needed to be performed (as discussed in Section 2.3.3) and thus was recorded frequently by a thermocouple in the wind tunnel's contraction.

\section{$2.3 \quad$ Hot-Wire Anemometry}

\subsubsection{Hot-Wire Probes}

Measurements of the mean and turbulent velocities in the boundary layer were accomplished using four-sensor hotwire anemometer probes shown in Figure 2.9. These probes were manufactured by Auspex Corporation and based on the type AVOP-4-100 model. Sensors are arranged as two orthogonal X-wire arrays with each sensor inclined a nominal $45^{\circ}$ from the probe axis. The four sensors are etched tungsten wire $5 \mu \mathrm{m}$ in diameter and approximately $1.2 \mathrm{~mm}$ in length equating to a nominal measurement volume of $0.75 \mathrm{~mm}^{3}$. Further information on these probes can be found in Wittmer (1998). 


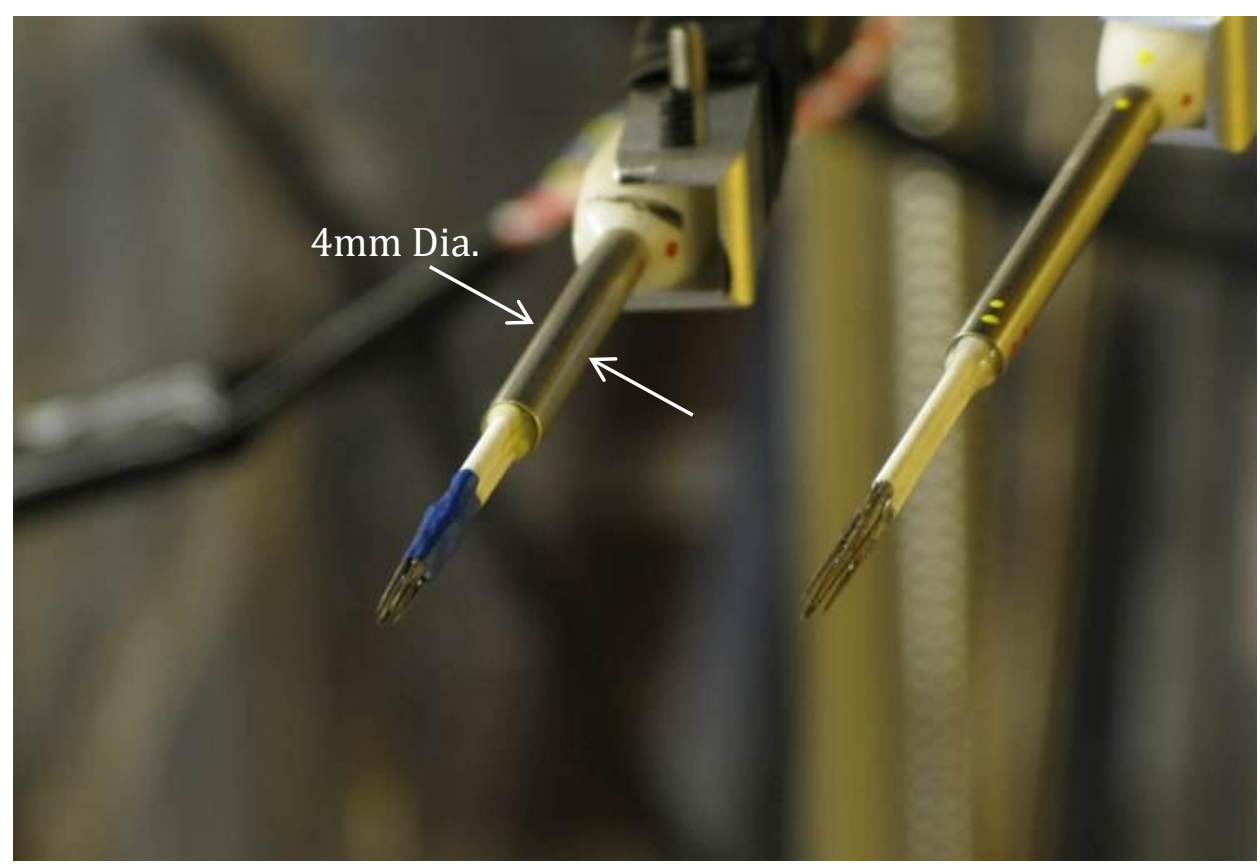

Figure 2.9. Four-sensor hotwire anemometer probes

\subsubsection{Constant Temperature Anemometers}

The four-sensor (quad-wire) probes were operated using a Dantec Dynamics Streamline Constant Temperature Anemometry System capable of automatically optimizing the sensor signal. This system generates a flat frequency response up to $18 \mathrm{kHz}$, contains a built in signal conditioner, and amplifier. The anemometer signals were passed through an Agilent E1432 16-bit, 64-channel digitizer and signal analyzer controlled by a laptop computer.

\subsubsection{Quad-wire Calibration}

In order to calibrate a quad-wire probe, two calibrations must be performed. First a velocity calibration is needed where the probe is placed in a jet of known velocity and the velocity is then varied. The voltage output is recorded at each velocity to find the relationship between the two using King's Law. In this study an initial velocity calibration was performed in a uniform jet. The second necessary calibration is an angle calibration. To do this the quad-wire probe is pitched and yawed over a range of angles from $-45^{\circ}$ to $45^{\circ}$ in a constant velocity jet. The relationship between the flow angle and the velocities is determined by comparing the sensor outputs at known pitch and yaw angles through the use of a look up table (Wittmer et al. 1998). Angle calibrations for each probe were performed once prior to any measurements while velocity calibrations were performed frequently to account for temperature changes in the tunnel. Velocity calibrations were performed in the free stream of the wind tunnel test section in between measurements.

\subsection{Probe Mounting and Positioning}

\subsubsection{Traverse System}

In order to measure two-point correlations in the flow, two separate traverse systems were installed as seen in Figure 2.10. A traverse capable of travelling in three 
dimensions was mounted inside the test section and another traverse capable of travelling in one dimension was mounted in the starboard chamber. Both traverse systems were operated by a laptop computer and controllable down to movements of $0.025 \mathrm{~mm}$. Mounted to the single axis traverse is a 0.41 meter long $9.5 \mathrm{~mm}$ diameter rod that travels through the Lexan wall and into the test section. As shown in Figure 2.11, the three axis traverse consisted of two streamwise rails that mount to the ceiling panels of the test section, another two that mount to the floor panels, and two vertical rails. A horizontal rail connects the two vertical rails and is used to mount measurement instrumentation. Mounted on the horizontal rail are two 1.0 meter beams that extend in the streamwise direction and hold a 0.46 meter long rod that extends toward the wall for probe mounting. An active damping device was connected to one of the 1.0 meter support beams to reduce any vertical vibrations that might occur. Fully installed, the traverse systems created approximately an $11 \%$ blockage in the test section requiring higher fan speeds for desired test section flow velocities.

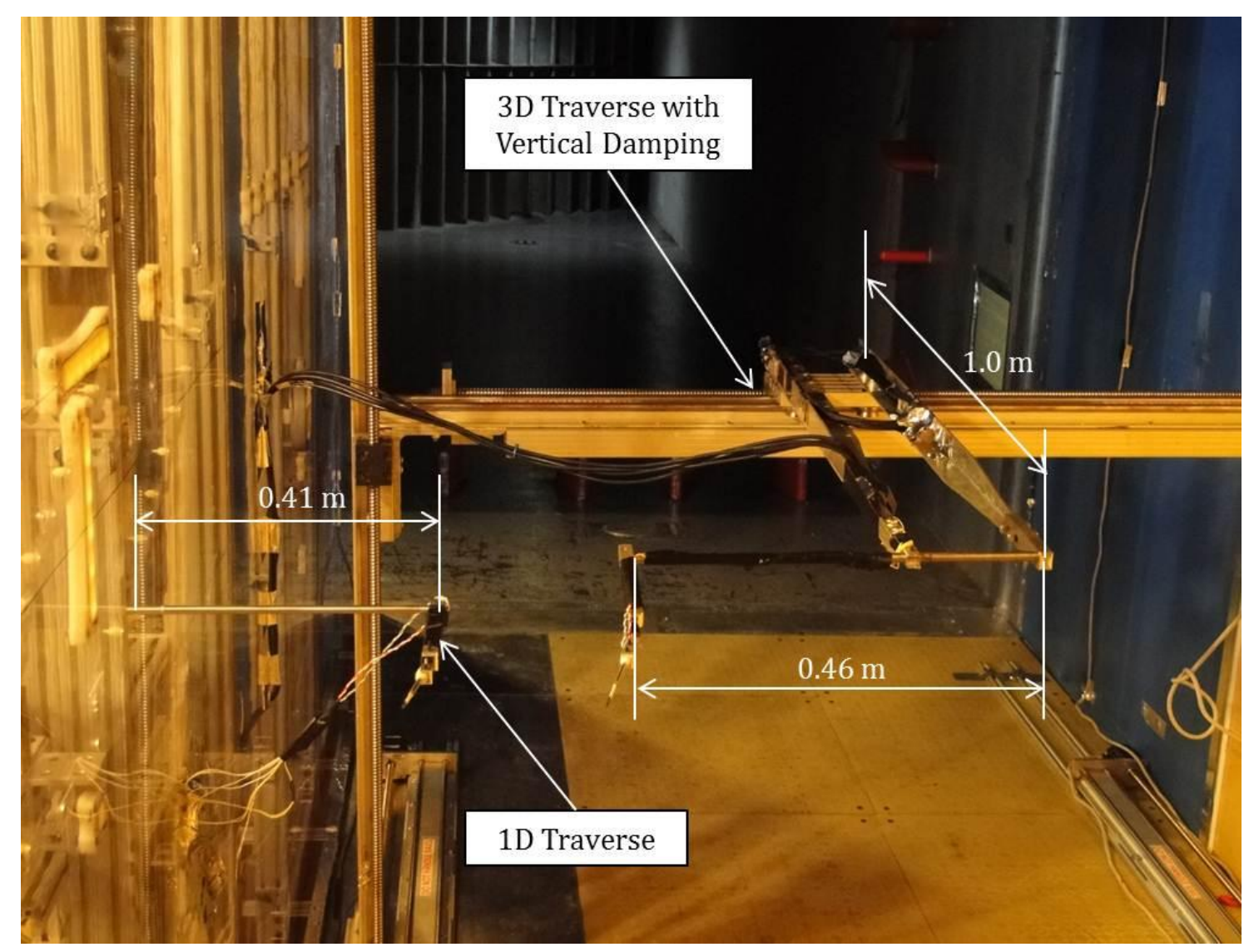

Figure 2.10. Three axis and single axis traverse with probes mounted. 


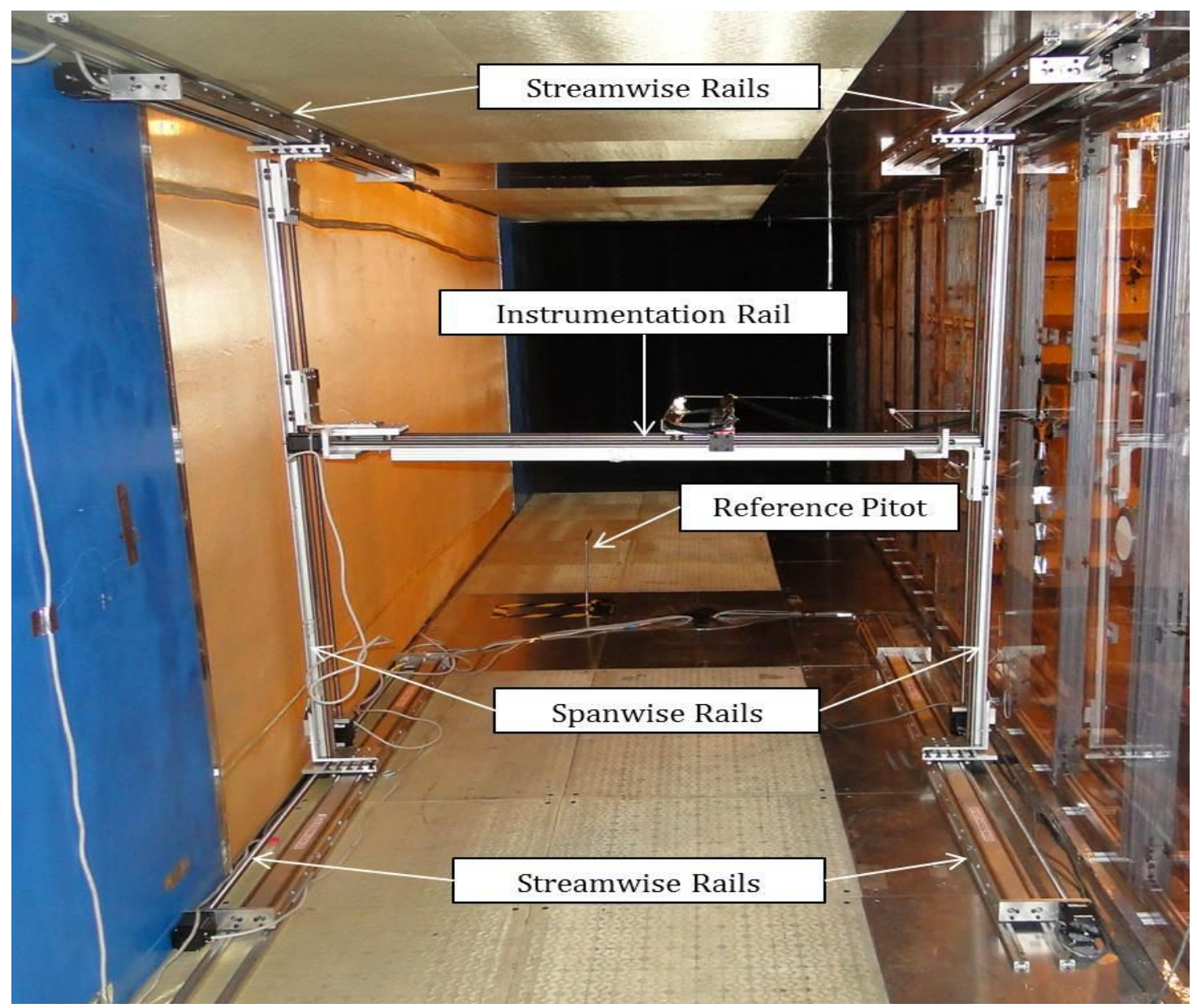

Figure 2.11. Three dimensional traverse system viewed from downstream.

\subsubsection{Laser Displacement Sensor}

The quad-wire probes were positioned using a Micro-Epsilon optoNCDT-1300-50 laser displacement sensor. This sensor, as seen in Figure 2.12, was mounted in the starboard chamber $32 \mathrm{~mm}$ from the Lexan wall. A hole was drilled through the Lexan to remove any interference with the laser. The sensor has a measurement range of $50 \mathrm{~mm}$ starting at a distance of $45 \mathrm{~mm}$ and a resolution of $10 \mu \mathrm{m}$. It works by using the principle of optical triangulation to output a voltage based on the distance from the reflecting surface to the sensor. An LED on the sensor indicates when the measurement is within its measurement range, out of range, or not receiving a clear signal. Calibrating the laser displacement sensor before locating each probe was necessary to determine the linear relationship between distance and voltage output. The calibration was performed using gage blocks to create a reliable and repeatable method. 


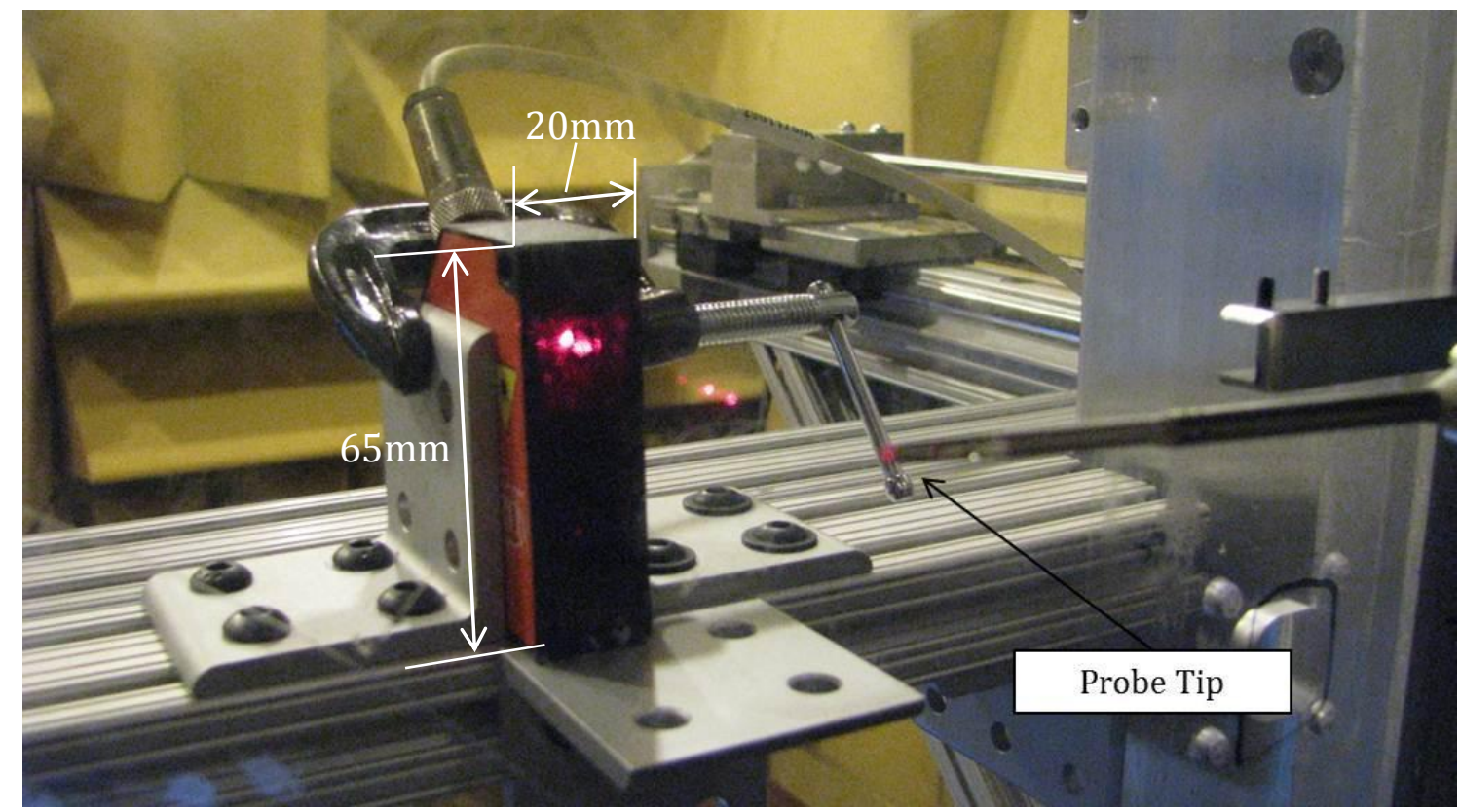

Figure 2.12. Laser displacement sensor

\subsubsection{Probe Separation}

The probe setup used to measure all boundary layer and two-point correlation statistics can be seen in Figure 2.13. In order to achieve a minimum probe separation of 4 $\mathrm{mm}$ in both the streamwise and normal-to-wall directions, governed by the measurement grids discussed in section 2.5, each probe needed to be pitched and yawed. Probe angles were determined using a laser pointer inserted into a rapid prototyped plastic that fit directly into the probe holders. The laser was directed onto a sheet of grid paper that was aligned with the axes of the test section and taped to the wall. By moving the three axis traverse a known distance in the normal-to-wall direction and recording the laser's initial and final locations on the grid paper the pitch and yaw angles could be calculated. In this experiment positive pitch corresponded to the probe tip moving in the positive y direction and positive yaw to the positive $\mathrm{z}$ direction. The probe mounted on the single axis traverse remained at a yaw angle of -5.67 degrees and a pitch angle of 4.00 degrees throughout all measurements. The probe on the three axis traverse varied between yaw angles of -13.35 and -13.82 degrees and pitch angles of -2.76 and -5.11 degrees. Visually inspecting the probes during testing showed that each probe occasionally vibrated in the $y$-direction a distance of no more than one prong diameter $(0.1 \mathrm{~mm})$. 


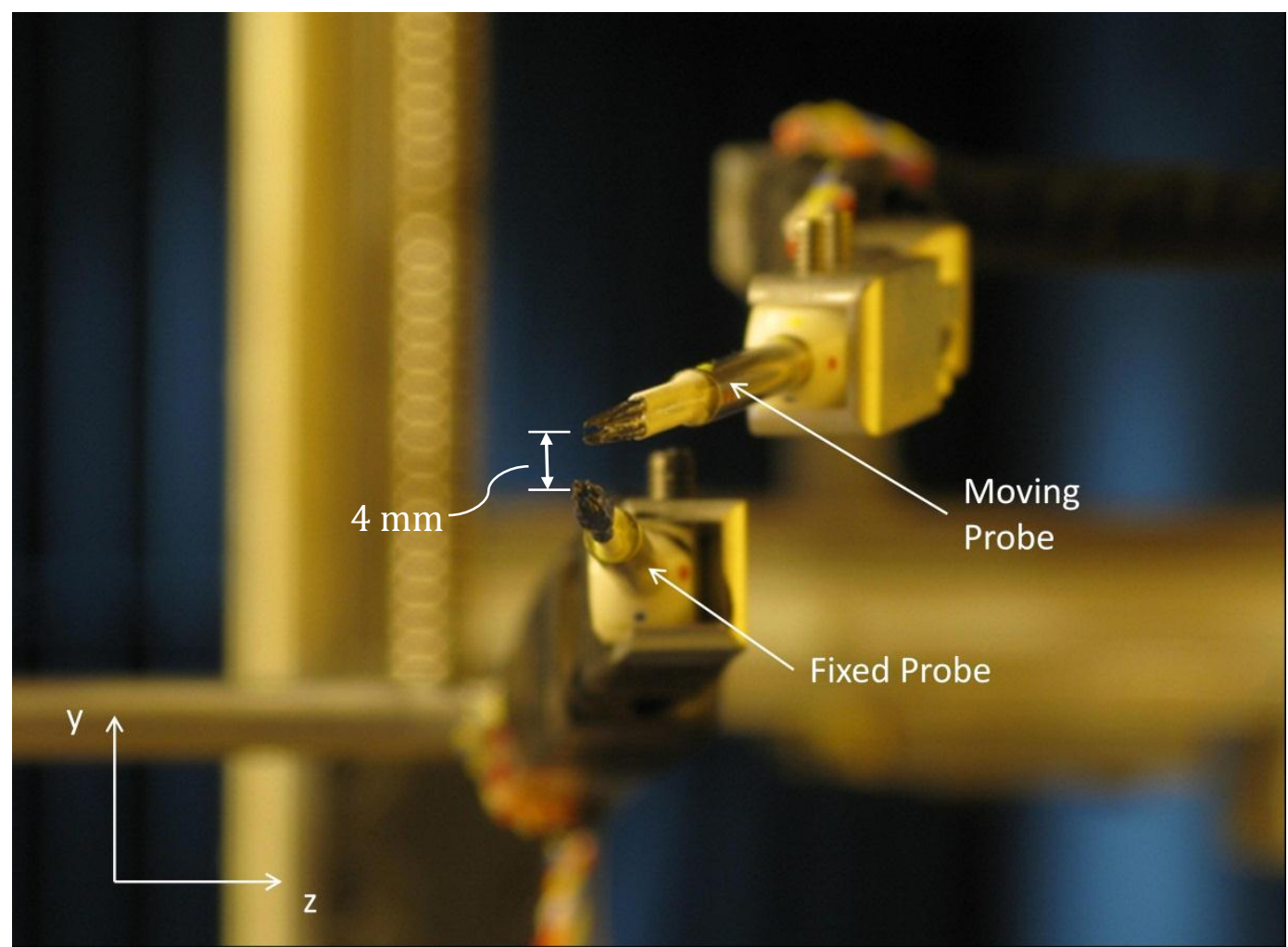

Figure 2.13. Quadwire probes at their minimal $y$ separation of $4 \mathrm{~mm}$ (from center to center of measurement volumes).

\section{$2.5 \quad$ Two-Point Quadwire Measurement Grids}

Measurement grids were designed using space time correlation estimates based on channel flow simulations by Moser et al. (1999) and adaptive traversing techniques developed to minimize the necessary measurement time. Although Moser's direct numerical simulation is for channel flow, it is reasonable to expect sufficient similarity in the boundary layer along the false wall in the Stability Wind Tunnel. Using the correlation function from Moser, a three dimensional grid was designed to get a high resolution of points where the correlation was largest. This three dimensional grid consisted of 31 fixed probe locations each of which included the moving probe measuring a cross section of 31 points in $z$ and 14 in $y$ totaling 13,454 points. The grid was then divided into 31 subsets each of which corresponded to a unique fixed probe location. To simplify the traversing and reduce the possibility of a collision between the probes, the grids were modified so the moving probe would never need to travel closer to the wall than the fixed probe. This required more fixed probe locations which consequently increased the number of necessary subsets to 59. Assuming that the flow is homogenous in the $y$-direction these subsets can be reduced by taking advantage of the relationship between the fixed and moving probes. If the moving and fixed probes switched their respective $z$-positions and remained at the same $y$-separation, the new correlation between the probes would be a mirror image of the initial correlation. This allowed the total measurement to be performed by measuring only 31 grids at 31 fixed probe locations totaling 1,781 points.

Figure 2.14 shows one of the 31 grids used to measure the four-dimensional twopoint space time correlation superimposed on an estimate of the zero time delay 
correlation based on Moser et al.'s (1999) DNS channel flow simulation. The black dots refer to moving probe measurements and the green refer to measurements previously performed by other measurement grids. To further reduce the necessary measurement time adaptive traversing techniques were used. This was done by having the two-point measurement code monitor the coherence between velocity signals from the two probes and decide when measurements at greater separation were no longer relevant. Since determining the full two-point space time correlation was the principal objective of this study, all 31 grids were measured continuously spanning a total of 18 hours. This ensured that all velocity calibrations were similar and probe positioning was consistent for each grid.

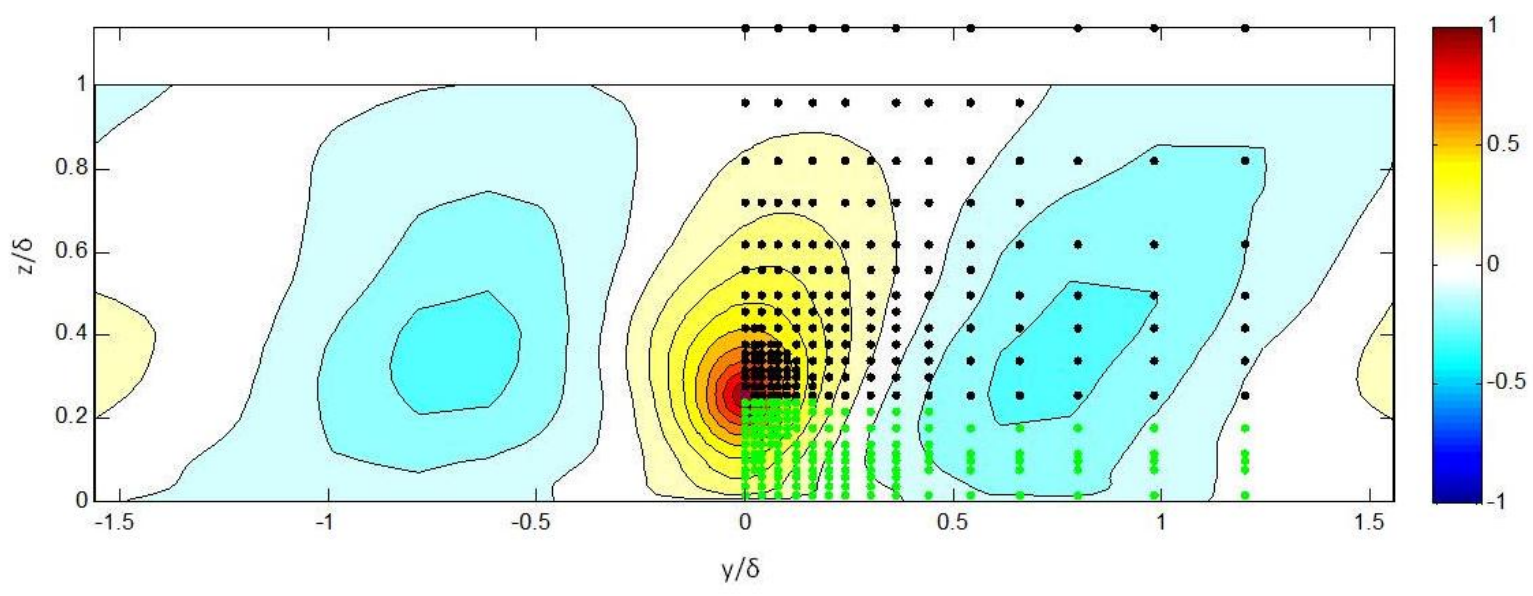

Figure 2.14. One of 31 two-point grids used in measuring the four-dimensional two-point space time correlation, superimposed on an estimate of the zero time delay correlation based on Moser et al.'s (1999) DNS channel flow simulation 


\section{CHAPTER 3 Analysis}

\subsection{Measurement Summary}

The main goal of this study is to fully document the two-point space time correlation function and use it to predict the sound generated by a rotor. Therefore, the pressure gradient was measured to position the wall in order to achieve a zero pressure gradient flow as discussed in Section 3.2. Section 3.3 discusses the single point velocity measurements performed to determine the mean and fluctuating velocity characteristics of the boundary layer for a number of flow and trip configurations. Detailed two-point measurements were performed for a free stream flow of $30 \mathrm{~m} / \mathrm{s}$ with a single $9.5 \mathrm{~mm}$ trip and are discussed in Section 3.4. The correlation function from the two-point measurements is shown to be the source of the unsteady upwash spectrum for a hypothetical rotor embedded in the boundary layer in Section 3.5.1 which consequently determines the far-field sound pressure levels. Far-field sound predictions for a scaled version of the Sevik rotor interacting with this correlation are shown in Section 3.5.5.

\subsection{Pressure Gradient}

Mean wall pressure was measured at 24 locations along the floor and ceiling of the test section to obtain the pressure gradient along the test surface. This allowed the false wall to be adjusted to obtain a zero pressure gradient in the test section. The mean pressure when the wall was adjusted to its final position is shown in Figure 3.1. It can be seen that there is a slight pressure gradient towards the downstream end of the test section which is thought to be a result of boundary layer growth along the Kevlar wall on the port side of the test section. The slight gradient in the downstream section was unable to be removed due to physical restrictions in the test section. The wall was never re-adjusted since the pressure gradient remained unaffected by the change in flow velocity and trip configuration. Since all measurements for this study were performed at an $x$ location of 4.72 meters, the mean wall pressure gradient was assumed negligible. 


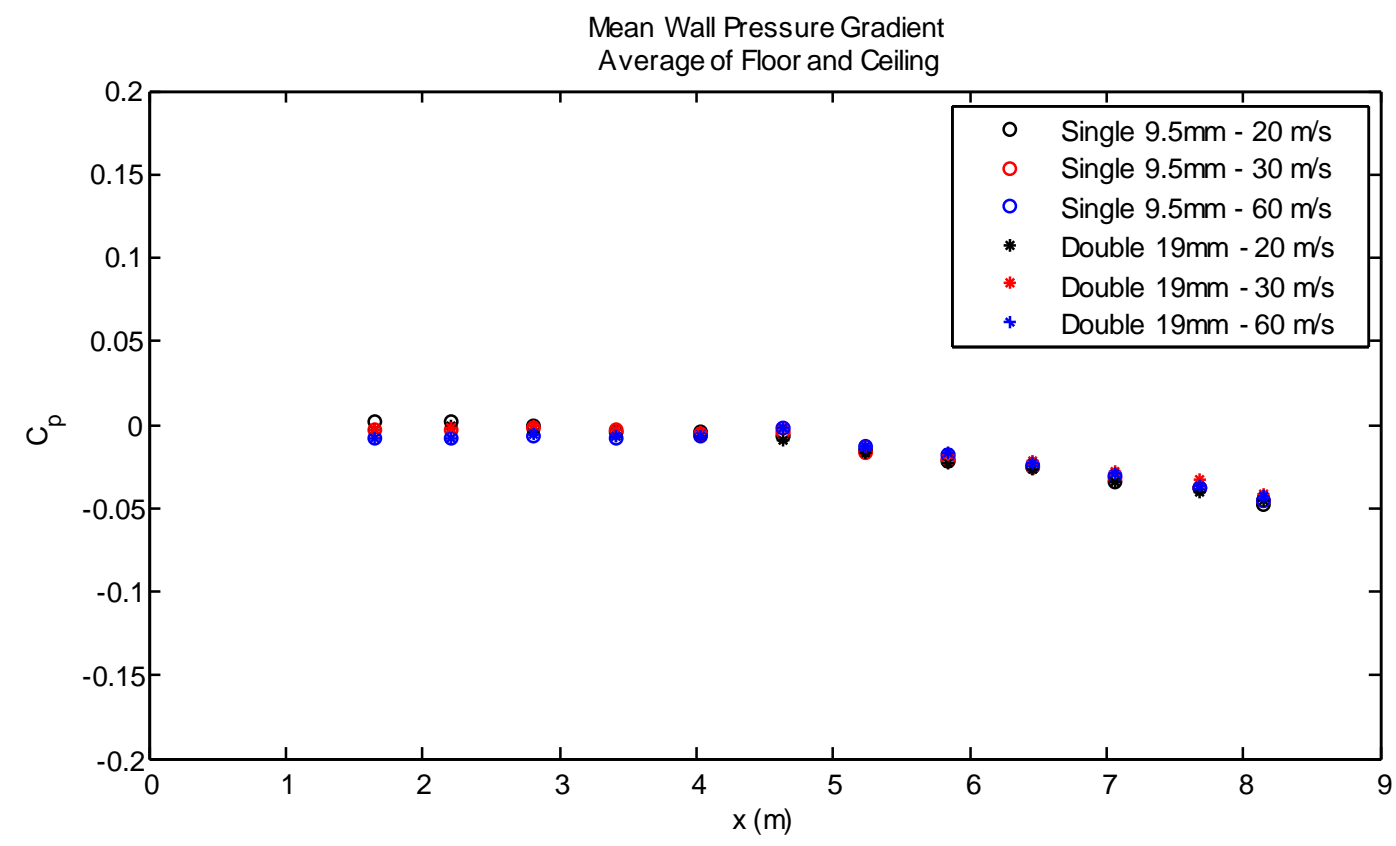

Figure 3.1. Mean pressure along the wall of the test surface

\subsection{Single Point Measurements}

\subsubsection{Mean Velocities}

Mean velocity measurements throughout the boundary layer were performed for free stream flow speeds of 20,30 , and $55 \mathrm{~m} / \mathrm{s}$ for both a single $9.5 \mathrm{~mm}$ trip and double $19 \mathrm{~mm}$ trip configuration. A table of the main boundary layer parameters can be seen in Table 1. Boundary layer thickness was defined as the distance from the wall where the flow was 99 percent of the free stream velocity. As can be seen in the table, boundary layer thickness ranged from .092 to .173 meters and decreased with an increase in free stream velocity as expected.

\begin{tabular}{cccccccccc}
$\begin{array}{c}\text { Trip } \\
(\mathbf{m m})\end{array}$ & $\begin{array}{c}\mathbf{U}_{\text {ref }} \\
(\mathbf{m} / \mathbf{s})\end{array}$ & $\mathbf{X}(\mathbf{m})$ & $\boldsymbol{\delta}(\mathbf{m})$ & $\mathbf{C}_{\mathbf{f}}$ & $\boldsymbol{\delta}^{*}(\mathbf{m})$ & $\boldsymbol{\Theta}(\mathbf{m})$ & $\mathbf{R e}_{\boldsymbol{\delta}}$ & $\mathbf{R e}_{\boldsymbol{\Theta}}$ & $\mathbf{H}$ \\
\hline 9.5 & 20 & 4.72 & 0.101 & 0.00275 & 0.0120 & 0.0094 & 122,360 & 11,457 & 1.27 \\
9.5 & 30 & 4.72 & 0.101 & 0.00258 & 0.0116 & 0.0092 & 182,890 & 16,607 & 1.26 \\
9.5 & 55 & 4.72 & 0.092 & 0.00245 & 0.0094 & 0.0077 & 301,330 & 25,035 & 1.23 \\
$2 \times 19$ & 20 & 4.72 & 0.173 & 0.00260 & 0.0188 & 0.0152 & 209,490 & 18,436 & 1.24 \\
$2 \times 19$ & 30 & 4.72 & 0.186 & 0.00245 & 0.0193 & 0.0159 & 345,660 & 29,475 & 1.22 \\
$2 \times 19$ & 55 & 4.72 & 0.164 & 0.00235 & 0.0162 & 0.0135 & 515,460 & 42,421 & 1.20
\end{tabular}

Table 1. Boundary Layer Parameters

The mean velocities normalized on the edge velocity $U_{e}$ and the boundary layer thickness $\delta$ can be seen in Figure 3.2. This figure shows profiles which are fairly typical of a fully developed zero pressure gradient two-dimensional boundary layer. All profiles with the exception of the $55 \mathrm{~m} / \mathrm{s}$ single $9.5 \mathrm{~mm}$ trip configuration demonstrate a boundary layer with approximately zero $v$ and $w$ velocities. The anomaly is believed to be caused by the increase in flow speed which thus caused the probe to yaw at a different angle than 
measured with the flow speed off. The rationale behind this is that the $20 \mathrm{~m} / \mathrm{s}$ run with the same trip configuration was performed with the exact same quad-wire probe and probe angle which resulted in data that needed to only be rotated by the measured pitch and yaw. The same rotation matrix was applied to the $55 \mathrm{~m} / \mathrm{s}$ run resulting in a small $w$ velocity of less than 3\% throughout the boundary layer which corresponds to a change in probe angle of less than 2 degrees. Verification of the single point quad-wire measurements was also achieved by a comparison to the two-point measurements and previous single hotwire measurements performed by Awasthi (2012) as shown in Figure 3.3.
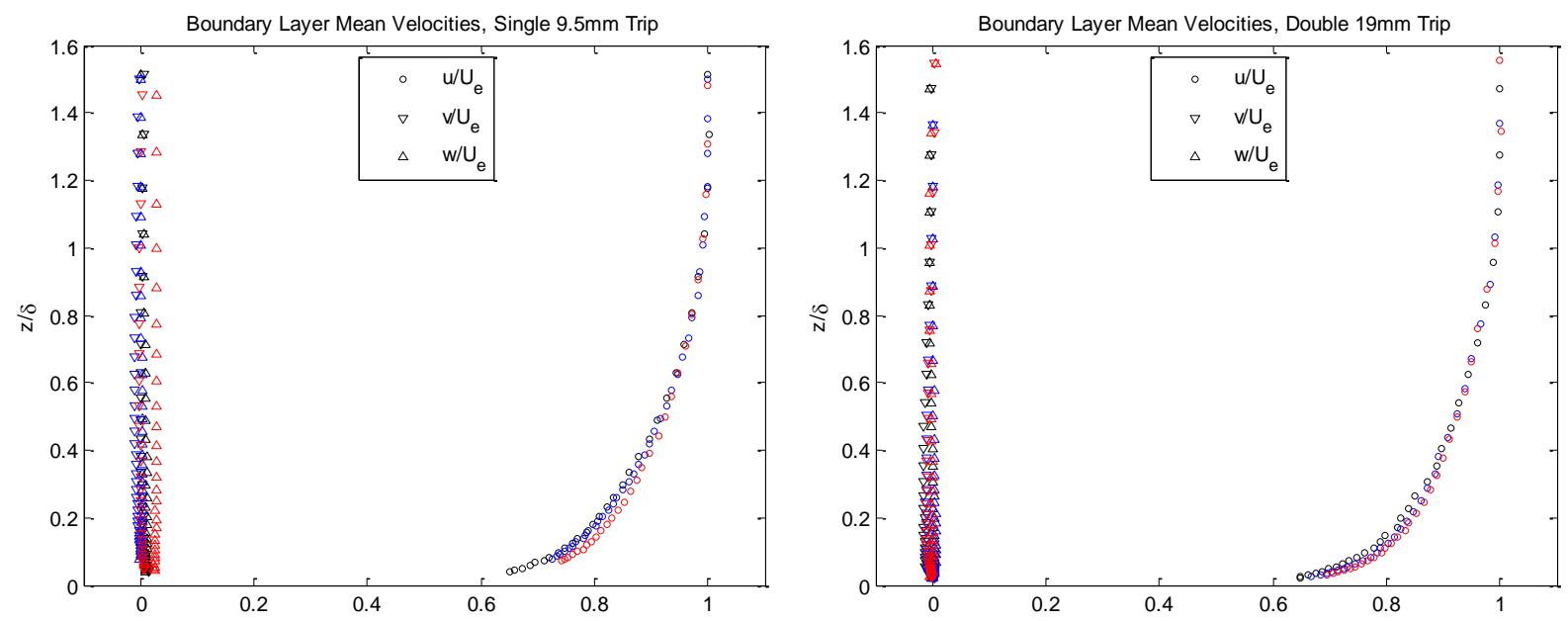

Figure 3.2. Mean velocity profiles. Black, blue, and red represent 20, 30, and $55 \mathrm{~m} / \mathrm{s}$ respectively.

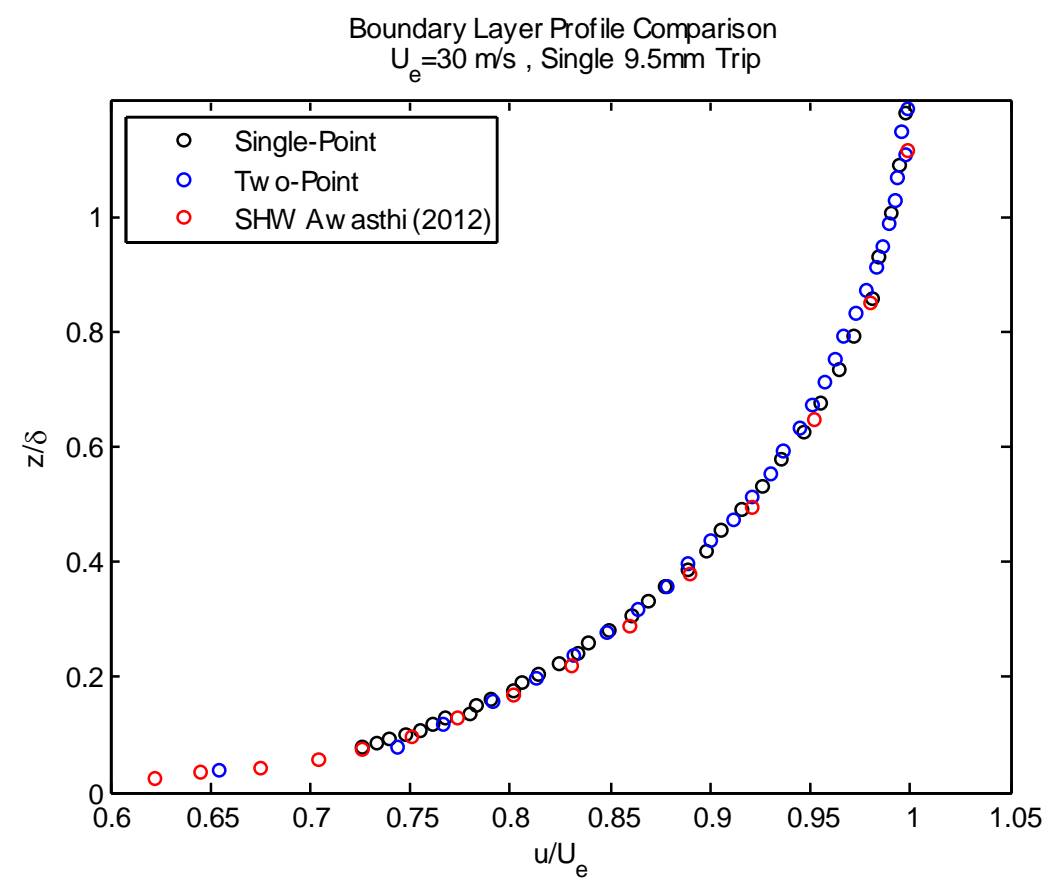

Figure 3.3. Mean velocity comparison between single-point quadwire, two-point quadwire, and concurrent study with single hot wire. 
Figure 3.4 shows the $u$ velocity profile for all six measurements. As can be seen, the outer part of boundary layer profiles normalized on $\delta$ and $U_{e}$ do not depend on flow speed or trip configuration suggesting that to some extent the statistics within the flow can be scaled to any of the desired configurations.

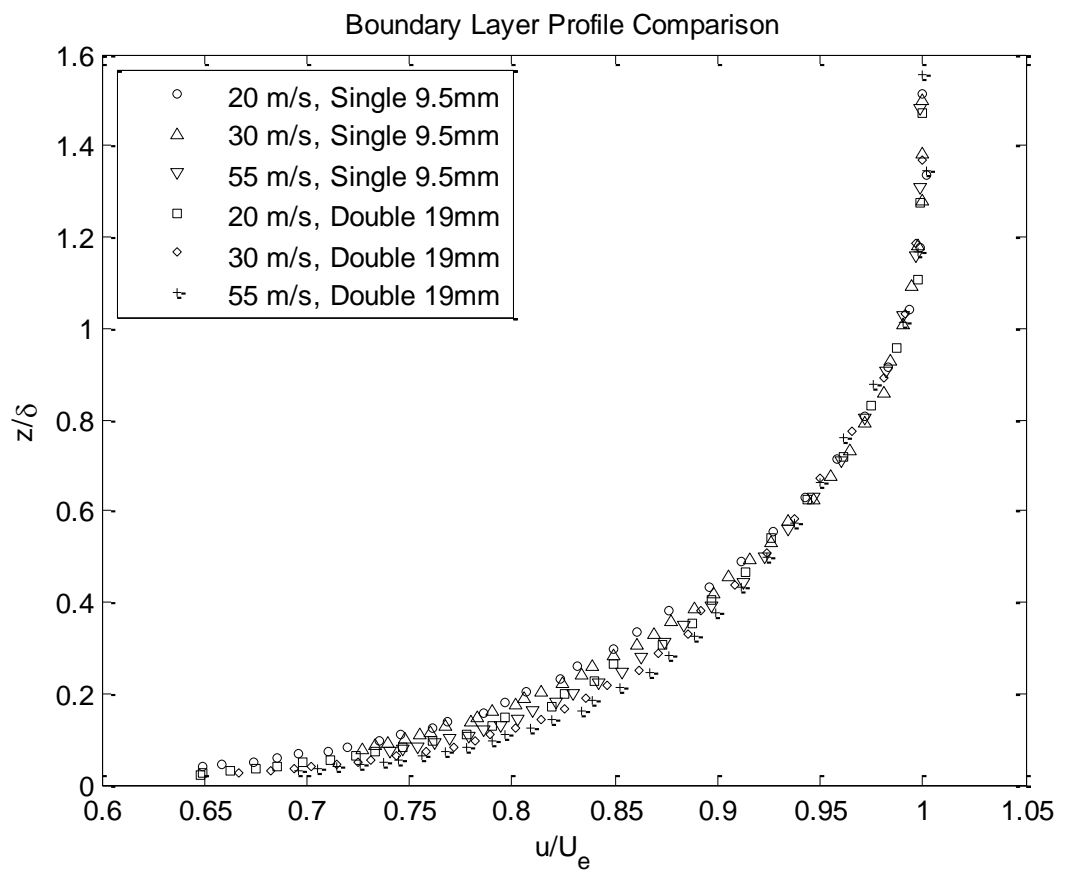

Figure 3.4. Boundary layer $u$ velocity comparison.

In order to check the two-dimensionality of the boundary layer, a cross section of the flow was measured in the $y z$-plane for a free stream velocity of $30 \mathrm{~m} / \mathrm{s}$ with the single $9.5 \mathrm{~mm}$ trip configuration. Figure 3.5 shows the resulting profiles from mid-span of the test section to 5 inches above mid-span in increments of $2.54 \mathrm{~cm}$. These profiles are similar enough to allow the flow to be treated as two-dimensional. 


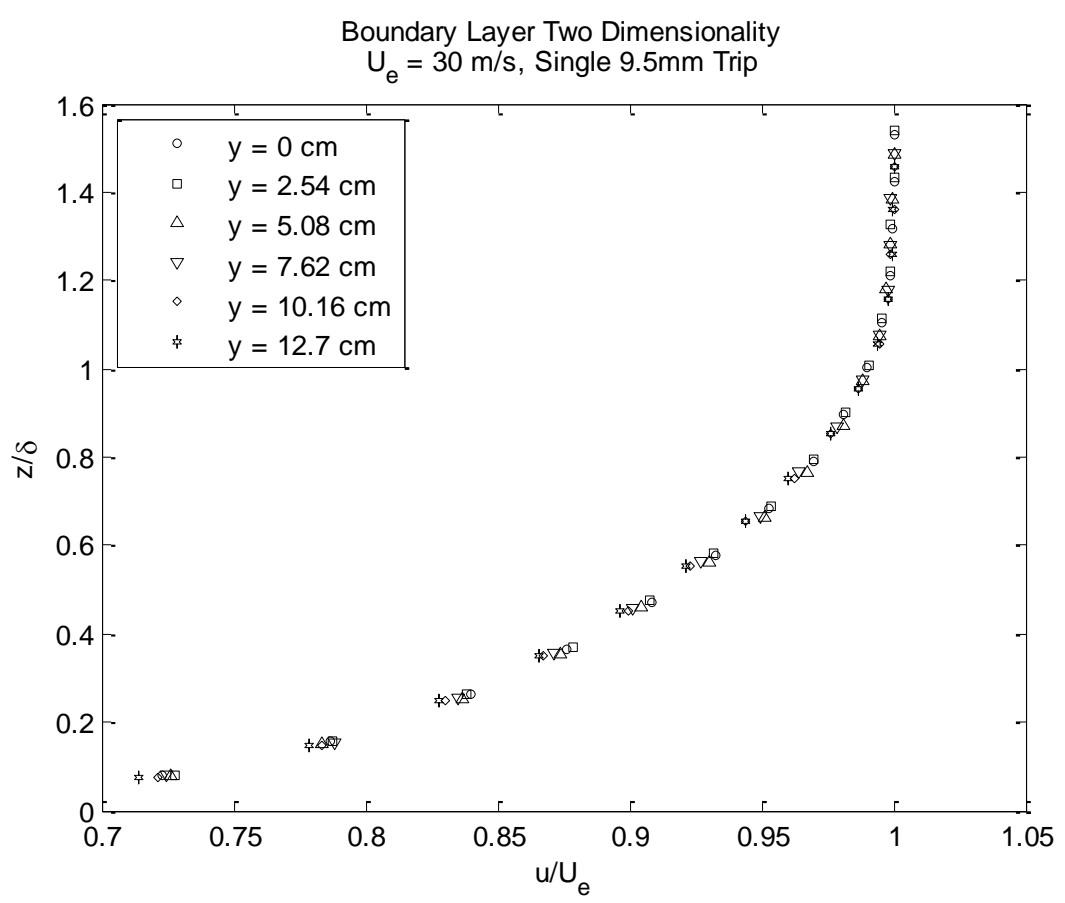

Figure 3.5. Boundary layer two-dimensionality

Boundary layer behavior near the wall was assumed to follow the law of the wall theory as described by Pope (2000), which states the velocity is a function of distance from the wall $z$, friction velocity $u_{t}$, and dynamic viscosity $v$ as defined by Equation 3.1.

$$
\frac{u}{u_{t}}=f\left(\frac{y u_{t}}{v}\right)
$$

The log law region is defined as Equation 3.2 where $B$ is a constant of 5.2 and $\kappa$ is the von Karman constant of 0.41 according to Pope. This region is typically valid from $z^{+}>30$ to about $20 \%$ of the boundary layer thickness.

$$
\frac{u}{u_{t}}=\frac{1}{\kappa} \ln \left(z^{+}\right)+B \quad, \quad z^{+}=\frac{z u_{t}}{v}
$$

Equation 3.2

This equation was used to predict the velocity profiles near the wall of the test section to compare to the experimental quad-wire data on a $u^{+}$vs $z^{+}$plot. Varying the experimental skin friction coefficient $C_{f}$ shifts the experimental data thus allowing the actual skin friction coefficient to be found by matching the experimental inner layer to the theoretical. These $C_{f}$ values are those listed in Table 1, a comparison with previous smooth wall studies can be seen in Figure 3.6. Figure 3.7 shows the law of the wall results for all six single point velocity profiles. 


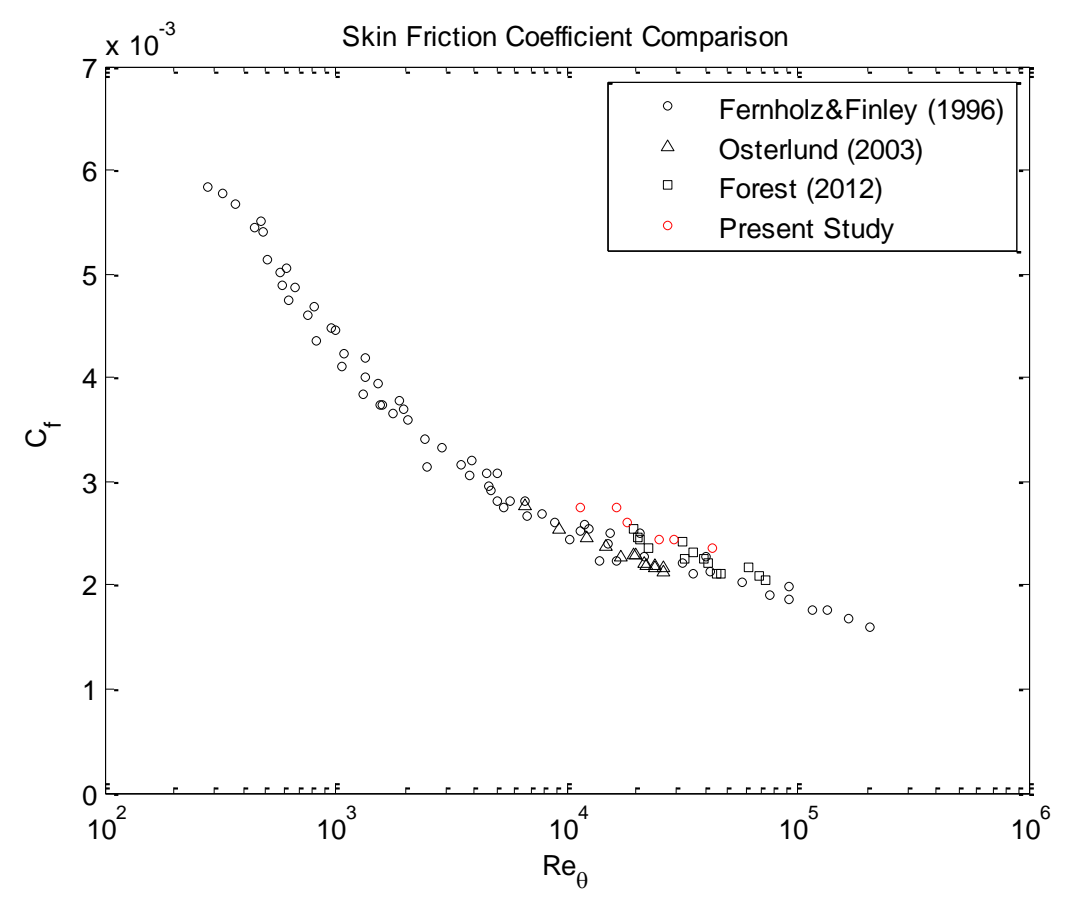

Figure 3.6. Skin friction coefficient comparison to previous smooth wall studies.

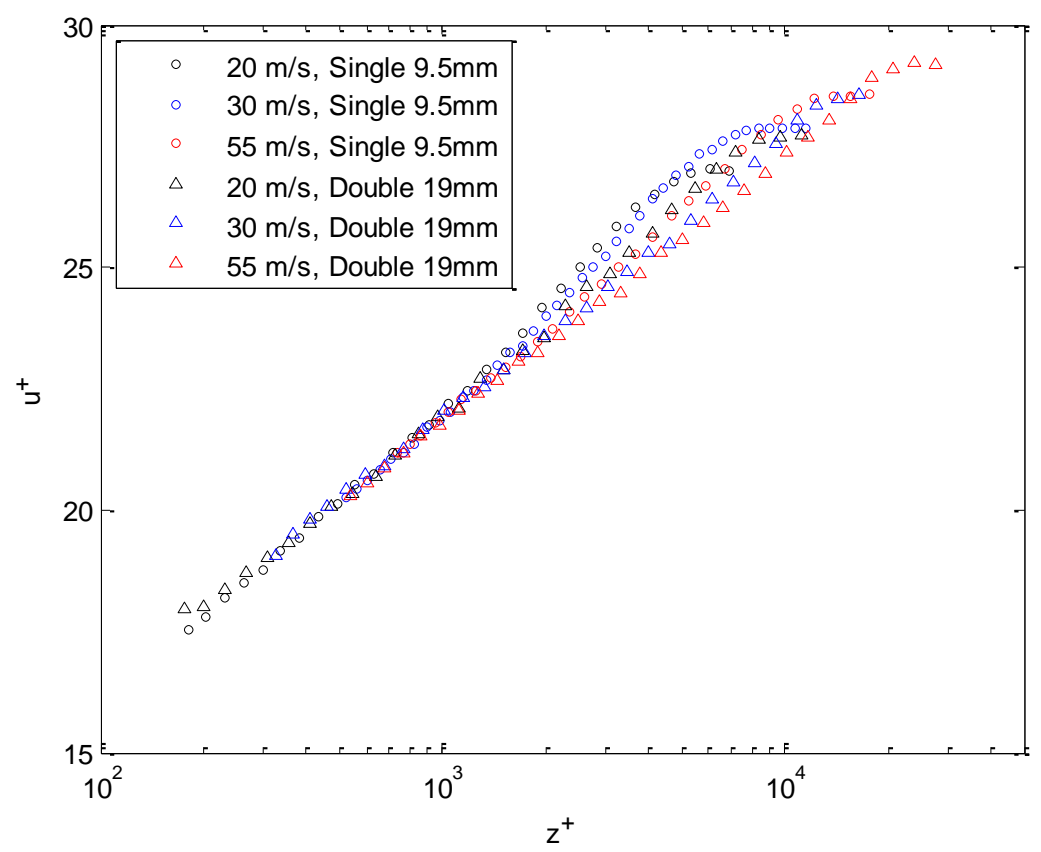

Figure 3.7. Law of the wall

\subsubsection{Boundary Layer Turbulence}

The root-mean-square (RMS) turbulent velocity fluctuations were also measured for each of the six configurations listed in Table 1. The data from these runs (with the exception of the 20 and $55 \mathrm{~m} / \mathrm{s}$ runs with the single $9.5 \mathrm{~mm}$ trip) required a probe angle transformation as discussed in Section 3.4.1 since the quadwire probe was at a large angle 
to the flow. The three turbulent normal stresses and the non-zero Reynolds shear stress $u w$ can be seen in Figure 3.8a. As would be expected $u^{2}$ is the largest of the three normal stresses and rises monotonically as the wall is approached (the finite size of the 4-sensor probe did not allow measurements close enough to the wall to reveal the maximum in this profile). Spanwise velocity fluctuations $v^{2}$ are about half of $u^{\prime 2}$, and normal to wall fluctuations $w^{2}$ slightly less than that, particularly close to the wall where normal fluctuations are suppressed by the non-penetration condition. The negative Reynolds shear stress does reach a clear maximum in the bottom $20 \%$ of the boundary layer thickness. These maximum values are quite consistent with the boundary layer skin friction coefficients inferred from the mean velocity profiles with the exception of the single $9.5 \mathrm{~mm}$ trip at $55 \mathrm{~m} / \mathrm{s}$ where changes in the probe orientation may have influenced the peak Reynolds shear stress. Figure 3.8b shows the same $u^{2}$ normal stresses but normalized on inner region variables instead of edge velocity. This scaling demonstrates a slightly better collapse in the data with the exception of two of the configurations. The $55 \mathrm{~m} / \mathrm{s}$ run with the single $9.5 \mathrm{~mm}$ trip installed is thought to have encountered a change in angle during testing causing the reprocessed data to be slightly skewed. Measurements at $20 \mathrm{~m} / \mathrm{s}$ with the double $19 \mathrm{~mm}$ trip were performed with a quadwire probe at a high angle relative to the flow thus requiring a transformation matrix to be applied as discussed in Section 3.4.1. Since this configuration was not measured with a probe at a low angle relative to the flow a transformation matrix could only be estimated thus resulting in the slightly higher $u^{\prime 2}$ values. 
(a)

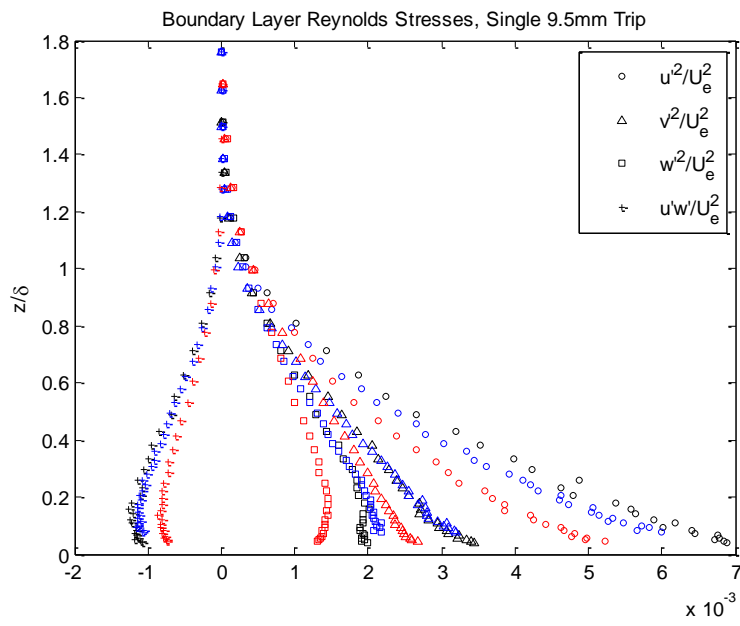

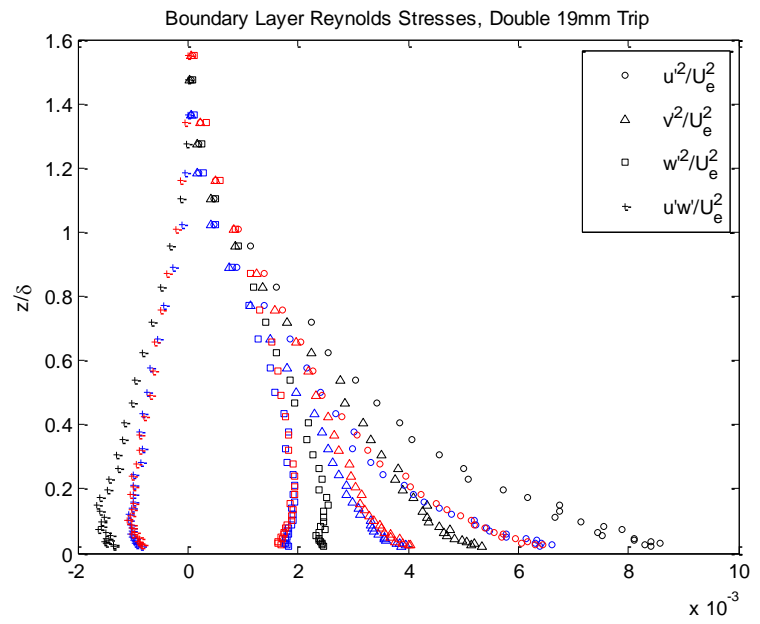

(b)

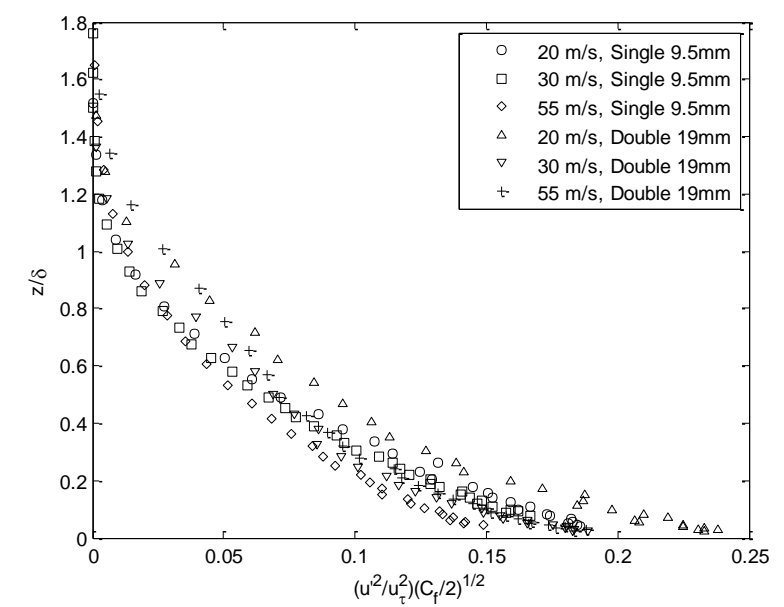

Figure 3.8. (a) Turbulent normal stresses and non-zero Reynolds stress profiles normalized on $U_{\mathrm{e}}^{2}$. Black represents $20 \mathrm{~m} / \mathrm{s}$, blue $30 \mathrm{~m} / \mathrm{s}$, and red $55 \mathrm{~m} / \mathrm{s}$. (b) $u^{\prime 2}$ normal stress normalized on inner region variables

Figure 3.9 shows autospectra of velocity measured at representative points throughout the boundary layer. At the boundary layer edge $(z / \delta=101 \%)$, the u-component spectrum shows a clear inertial subrange with a spectra slope of $-5 / 3$ and a roll-off at high frequencies in the dissipation range. The $v$ and $w$ component spectra are almost identical and show less intense velocity fluctuations than the $u$-spectra at low frequencies, and larger levels at high frequencies. At the highest frequencies the ratio between the $v$ or $w$ spectra and the $u$ spectra is close to 2 , as would be expected of isotropic homogeneous turbulence. Moving in towards the wall the overall level of the $u$ spectrum rises, but the spectral shape retains the same basic form. The $v$ and $w$ spectra, however, change substantially. Most notably the low-frequency levels of the $w$ spectrum fall at low frequencies as vertical large scale motions are suppressed by the non-penetration at the wall. As the wall is approached this suppression is seen at higher and higher frequencies. However, closest to the wall at $z / \delta=7 \%$, the $v$ and $w$ spectra still show isotropic behavior at the highest frequencies. 


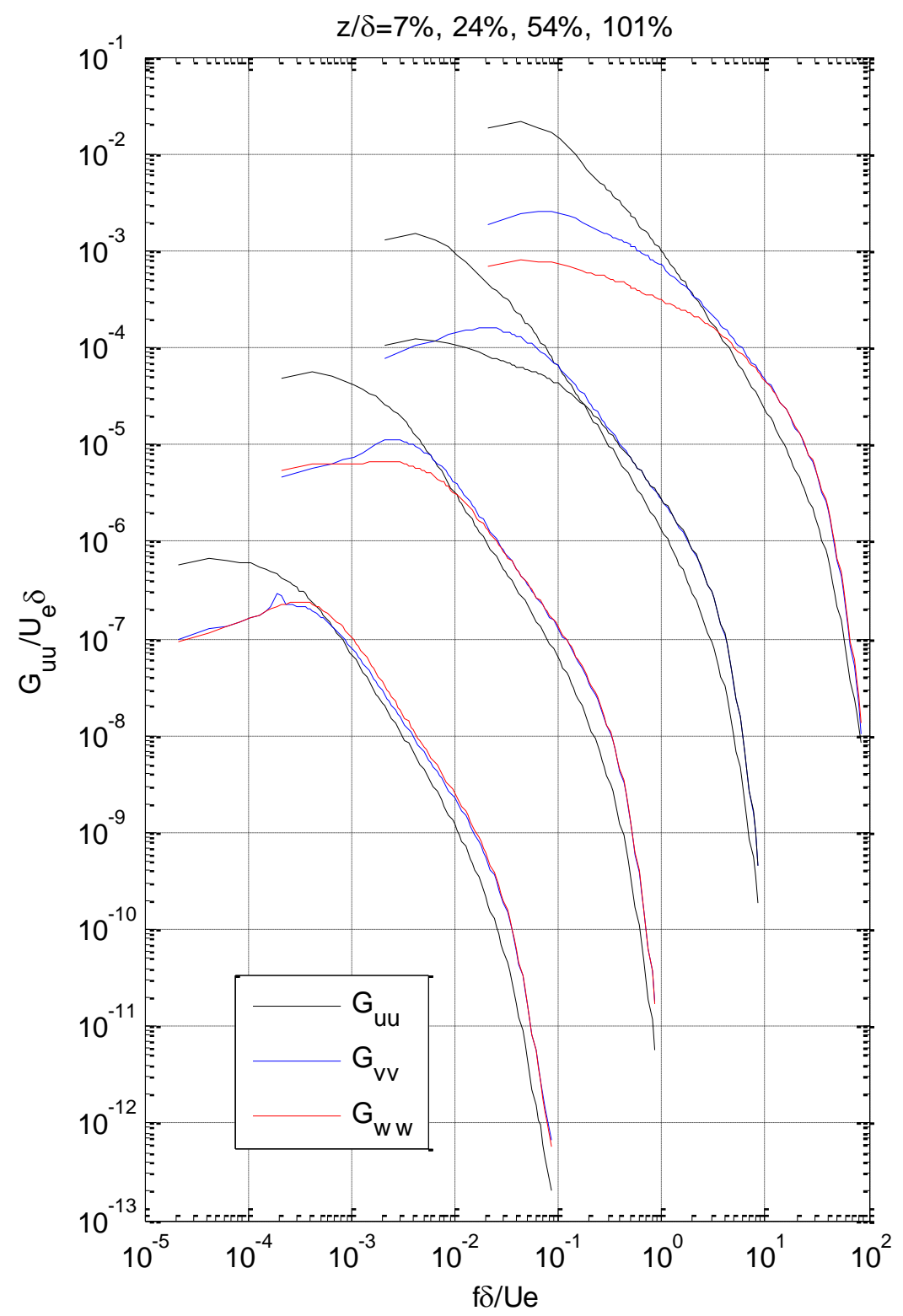

Figure 3.9. Autospectra of velocity fluctuations measured at various positions within the boundary layer. Spectral levels and frequencies are multiplied by 0.1 ,

0.01 and 0.001 for $z / \delta=24 \%, 54 \%$ and $101 \%$, respectively.

\subsection{Two-Point Measurements}

Two-point measurements were performed over a cross section located 4.72 meters downstream of the trip at a free stream velocity of $30 \mathrm{~m} / \mathrm{s}$ with the single $9.5 \mathrm{~mm}$ trip configuration using the measurement grids discussed in Section 2.5. When fully combined, these grids included over 1800 different relative positions in the boundary layer with a 
resolution of $4 \%$ of the boundary layer thickness. In this setup the fixed hotwire probe remained at a low yaw angle of $-5.67^{\circ}$ and low pitch angle of $4.00^{\circ}$. The moving probe was at a high yaw angle of $-13.8^{\circ}$ and low pitch angle of $5.11^{\circ}$ to allow for desired probe separation as discussed in Section 2.4.3.

\subsubsection{Probe Angle Transformation}

After applying the appropriate probe angle rotations to the data, errors appeared in the Reynolds stress tensors for runs with a probe yaw angle greater than 8 degrees. This encompassed all of the two-point and single point measurements with the exception of the 20 and $55 \mathrm{~m} / \mathrm{s}$ runs with the single $9.5 \mathrm{~mm}$ trip configuration. Although the mean velocity profiles measured by the fixed probe and moving probe at matching points in the boundary layer were equivalent, the Reynolds stress profiles were dissimilar as shown in Figure 3.10. To correct the error in the Reynolds stress tensors a transformation matrix was derived.
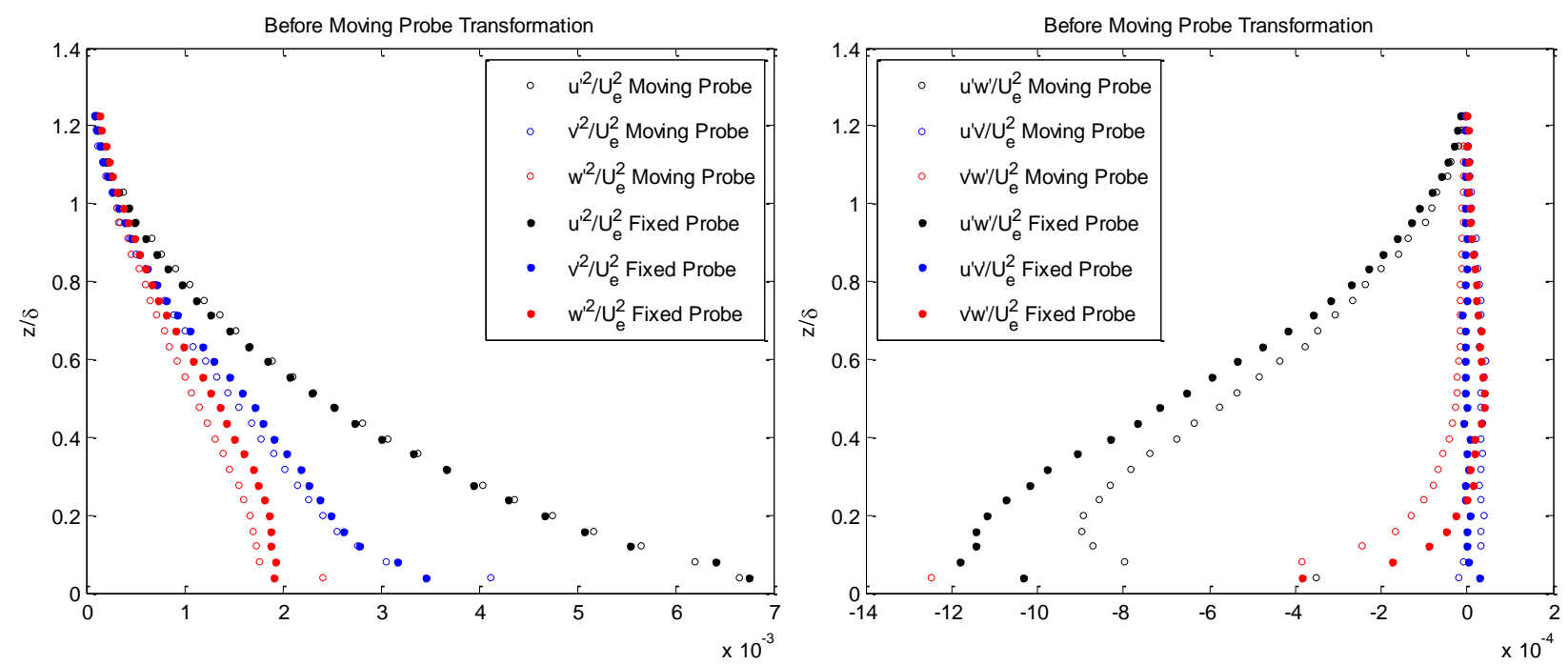

Figure 3.10. Reynolds stress profiles of the moving and fixed probe before applying a transformation to the moving probe.

Since the fixed probe was at a yaw angle of less than 4 degrees the measured Reynolds stress tensor was assumed correct. Also, since these flows are unidirectional only a single point was used from the angle calibration. Therefore, since the mean velocities are correct and consistent between the two probes, the local slope of the calibration surface must be slightly in error at the large probe angles. If the corrected moving probe fluctuating velocity components are denoted as $u_{T}^{\prime}, v_{T}^{\prime}, w_{T}^{\prime}$ and the measured fluctuating velocity components are denoted as $u^{\prime}, v^{\prime}, w^{\prime}$, then a transformation matrix can be found that relates the measured to the actual as given by Equation 3.3.

$$
\left\{\begin{array}{c}
u_{T}^{\prime} \\
v_{T}^{\prime} \\
w_{T}^{\prime}
\end{array}\right\}=\left[\begin{array}{lll}
T_{11} & T_{12} & T_{13} \\
T_{21} & T_{22} & T_{23} \\
T_{31} & T_{32} & T_{33}
\end{array}\right]\left\{\begin{array}{c}
u^{\prime} \\
v^{\prime} \\
w^{\prime}
\end{array}\right\}
$$

The $\boldsymbol{T}$ matrix can be determined by minimizing the difference between the fixed probe and moving probe stress profiles as previously performed by Borgoltz (2007). The resulting $\boldsymbol{T}$ matrix for the moving probe at a yaw angle of 13.8 degrees was found as 
Equation 3.4. This matrix is within a few percent of the identity matrix implying only small corrections to the calibration surface slope were needed. The resulting Reynolds stresses after the transformation matrix was applied can be seen in Figure 3.11. Although there were many more points used to determine the $\boldsymbol{T}$ matrix, only the average of these points at $31 \mathrm{z}$ locations are shown in the figure to reduce clutter.

$$
\boldsymbol{T}=\left[\begin{array}{ccc}
0.9816 & 0.0099 & -0.0483 \\
-0.0139 & 1.0299 & 0.0306 \\
-0.019 & 0.0131 & 1.0562
\end{array}\right]
$$

Equation 3.4
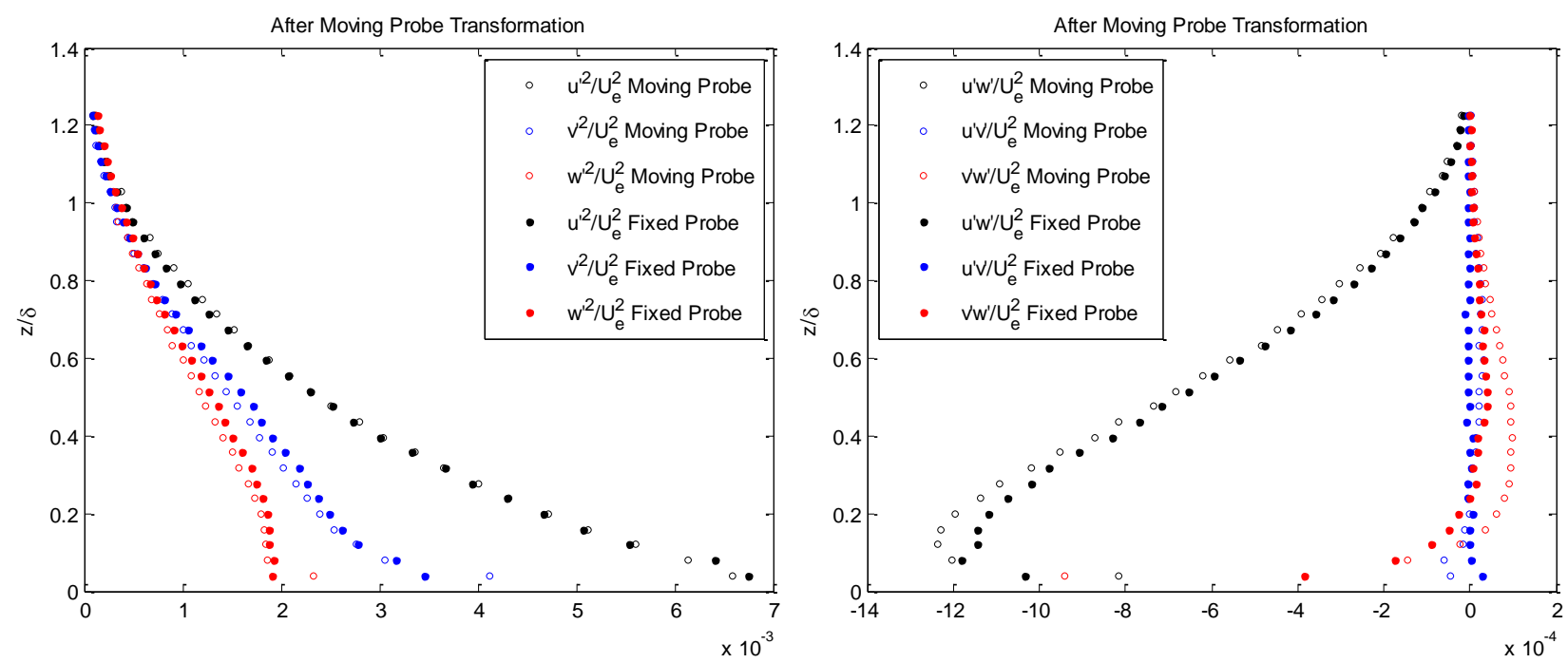

Figure 3.11. Reynolds stress profiles after applying a transformation to the moving probe.

\subsubsection{Two-Point Velocity Correlation}

The two-point velocity correlation tensor is defined as the expected value of the product of two velocity fluctuations as a function of space and time as shown in Equation 3.5 .

$$
R_{i j}\left(\boldsymbol{r}, \boldsymbol{r}^{\prime}, \tau\right)=\overline{u_{l}(\boldsymbol{r}, t) u_{\jmath}\left(\boldsymbol{r}^{\prime}, t^{\prime}\right)}
$$

Equation 3.5

The indices $i$ and $j$ range from 1 to 3 and correspond to the streamwise, spanwise, and normal-to-wall directions respectively. The position vectors of the two points in space are represented by $r$ and $r^{\prime}$ while the times are represented by $t$ and $t^{\prime}$ such that $t=t-t^{\prime}$ is the time delay. The correlation function at zero-time delay $(\tau=0)$ and zero separation $\left(r=r^{\prime}\right)$ reduces to the definition of the Reynolds stress tensor given by Equation 3.6.

$$
R_{i j}\left(\boldsymbol{r}, \boldsymbol{r}^{\prime}, 0\right)=\overline{u_{\imath}(\boldsymbol{r}, t) u_{\jmath}(\boldsymbol{r}, t)}
$$

Equation 3.6

Also, the correlation function has symmetry in time delay given by Equation 3.7.

$$
R_{i j}\left(\boldsymbol{r}, \boldsymbol{r}^{\prime}, \tau\right)=R_{j i}\left(\boldsymbol{r}, \boldsymbol{r}^{\prime},-\tau\right)
$$


The correlation function for this experiment can be more easily understood when expressed in terms of the normal to wall $R_{i j}\left(z, z^{\prime}, \tau\right)$ and spanwise $R_{i j}\left(\Delta y, z=z^{\prime}, \tau\right)$ coordinate systems.

The two-point space time correlation tensor is obtained by performing an inverse Fourier transform on the spectral data. The correlation function is defined as Equation 3.8 where $R_{i j}$ is the correlation tensor, $G_{i j}$ is the velocity spectrum tensor, $f$ is the frequency, and $\tau$ is the time delay.

$$
R_{i j}\left(\Delta y, z, z^{\prime}, \tau\right)=\int_{0}^{\infty} G_{i j}\left(\Delta y, z, z^{\prime}, f\right) e^{2 \pi i f \tau} d f
$$

Spectral data was first interpolated from 111 unequally spaced frequencies to 4096 equally spaced frequencies from 0 to $20.48 \mathrm{kHz}$. The auto-correlations of the fixed probe and moving probe were then found using Equation 3.8 through Inverse Fast Fourier Transform (IFFT). The $z=z^{\prime}$ terms at zero spanwise separation $(\Delta y=0)$ of the crosscorrelation matrix were obtained by averaging the auto-correlation of each of the two probes. To reduce the size of the resulting data file the correlation function was then interpolated from 4096 equally spaced times to 129 unequally spaced times. To validate this method the Reynolds stresses were extracted from the reduced correlation matrix, by use of Equation 3.6, and plotted against the non-processed data as seen in Figure 3.12. The reduction is shown to have no effect on the Reynolds stresses.

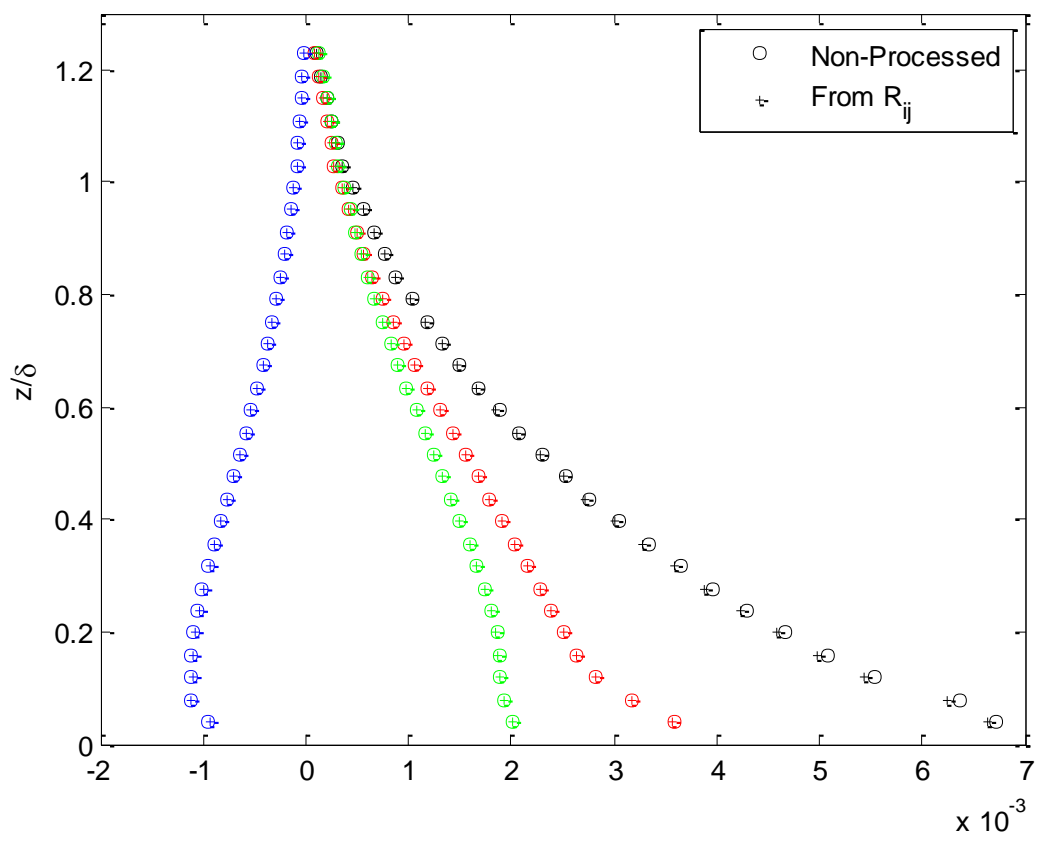

Figure 3.12. Comparison of Reynolds Stress Profiles from Non-Processed Data and Zero-Time Delay $R_{i j}\left(z=z^{\prime}\right)$. Black represents $u^{2}$, red $v^{2}$, green $w^{2}$, and blue $u w$.

\subsubsection{Normal-to-Wall Time Delay Correlation}


The first velocity correlation to be considered is the correlation at zero time delay and zero spanwise separation as shown in Figure 3.13. Correlation function at zero time delay and zero spanwise separation One of the main reasons to consider this cut through the correlation function is at zero time delay the diagonal of each plot $\left(z=z^{\prime}\right)$ represents the Reynolds stress tensor. Looking at the diagonal of the $R_{11}$ correlation at zero time delay starting from the bottom left it shows that the Reynolds normal stress $u^{\prime 2} / U_{e}^{2}$ starts at its maximum closest to the wall and monotonically decreases to zero which directly corresponds to single quadwire measurements as discussed in Section 3.3.2. Similar Reynolds stress profiles can be seen along the diagonal of the plots of $R_{22}, R_{33}$, and $R_{13}$. Off of the diagonal these maps show the extent of the normal to wall correlations. The correlation is dominated by the streamwise component $R_{11}$ as characterized by the more intense and wider spread contours. This component creates a half of an eye-shape meaning the boundary layer correlates more over greater separations closer to the wall than at further distances. This is not true for the other three relevant components $R_{22}, R_{33}$, and $R_{13} . R_{22}$ and $R_{33}$ exhibit correlation over greater separations closer to the middle of the boundary layer which is represented by the full eye-shape. Shown in $R_{22}$ there is an anti-correlation between spanwise velocity correlations close to the wall and in the rest of the bottom half of the boundary layer. The normal to wall velocity correlations $R_{33}$ show a large-scale positive correlation that extends well beyond the boundary layer edge. Overall this means that the structures inside the boundary layer responsible for the spanwise and normal-towall velocity fluctuations tend to be small in the normal-to-wall direction while the structures responsible for the streamwise fluctuations are larger and tend to be closer to the wall. The cross correlation terms $R_{12}$ and $R_{23}$ show no significant correlation at zero time delay as expected since the flow is homogeneous in the spanwise direction. 


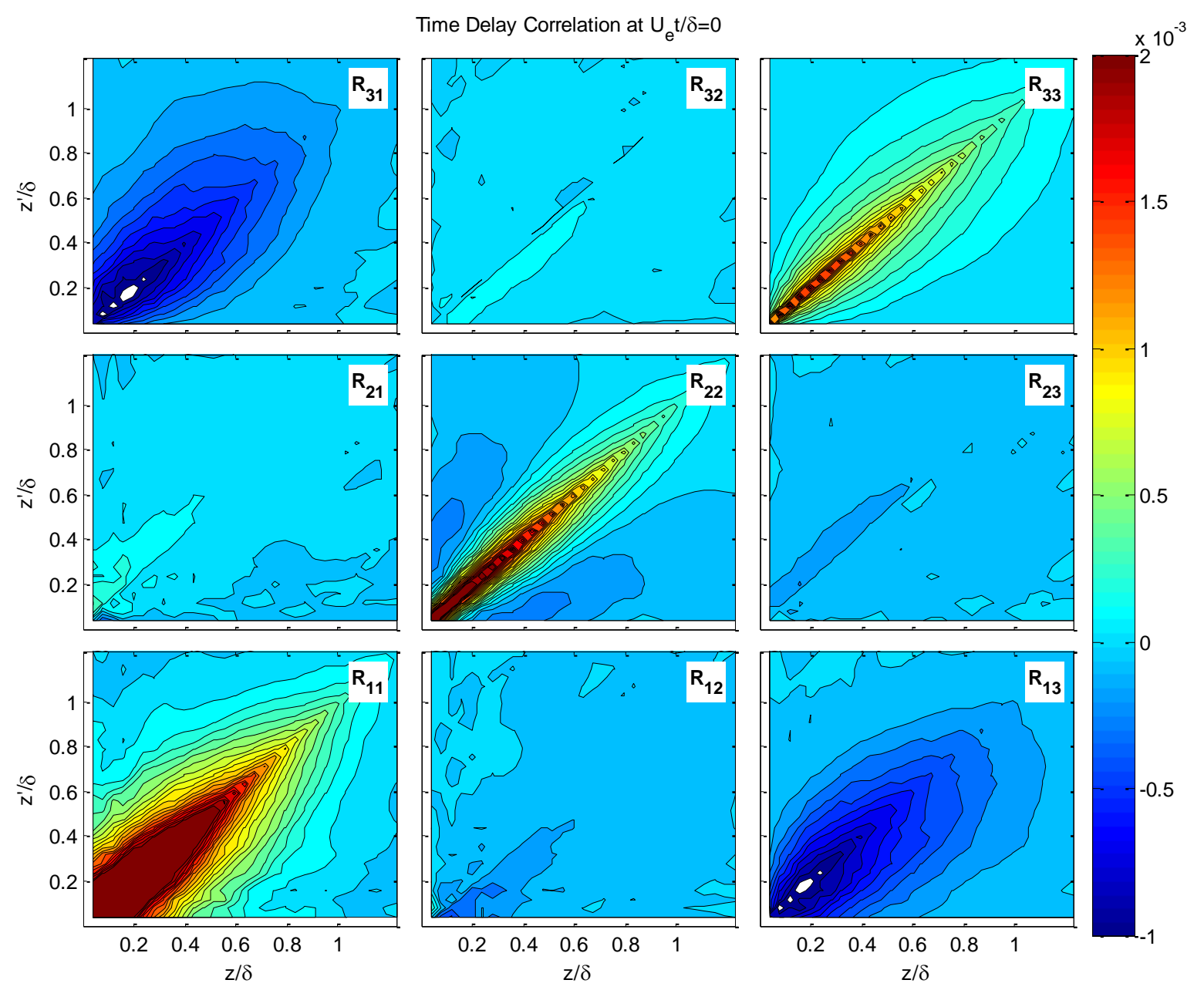

Figure 3.13. Correlation function at zero time delay and zero spanwise separation, $R_{i j}\left(0, z, z^{\prime}, 0\right)$

The next velocity correlation considered is the normal-to-wall correlation as it evolves over time. This is obtained by evaluating the correlation tensor at zero spanwise separation i.e. $R_{i j}\left(0, z, z^{\prime}, \tau\right)$ and can be seen in Video 1 . This video only shows the four dominant correlation coefficients. As the video progresses, the normal to wall distance of the fixed probe $(\mathrm{z})$ increases. The vertical axes represent the normal to wall location of the moving probe normalized on boundary layer. Time delay has been multiplied by the free stream velocity so the horizontal axis can be thought of as streamwise separation with the flow travelling from right to left. Streamwise velocity fluctuations again dominate the correlation function throughout the boundary layer. At small fixed probe distances from the wall (small $z / \delta$ ), the streamwise correlation coefficient $R_{11}$ demonstrates strong correlation in the inner half of the boundary layer which extends a large distance in the streamwise direction. Increasing the distance between the fixed probe and the wall shows that the streamwise velocity fluctuations exhibit a directional structure inclined from the wall. One would expect this directionality in the correlation to have a substantial effect on 
the timing of blade to blade lift correlations seen by a cutting rotor, and thus the radiated sound.

The spanwise velocity correlation $R_{22}$ also exhibits this highly directional structure which extends away from and toward the wall at approximately a 30 degree angle. Although the spanwise velocity fluctuations are similar to the streamwise fluctuations in directionality, the correlation is significantly less strong and does not extend nearly as far in the streamwise direction. Also present in the $R_{22}$ correlation maps are 2 negative lobes which develop downstream and below the inclined structure as well as just above and upstream. Normal to wall velocity fluctuations $R_{33}$ are less than the other two normal velocity fluctuations in both extent and magnitude. They demonstrate a nearly isotropic correlation throughout the boundary layer and typically extend over about $25 \%$ of the boundary layer thickness depending on the normal to wall fixed probe distance. The shear stress correlation $R_{13}$ shows a negative correlation which extends approximately 2 boundary layer thicknesses in the streamwise direction close to the wall and approximately 1 boundary layer thickness at greater normal to wall fixed probe locations.

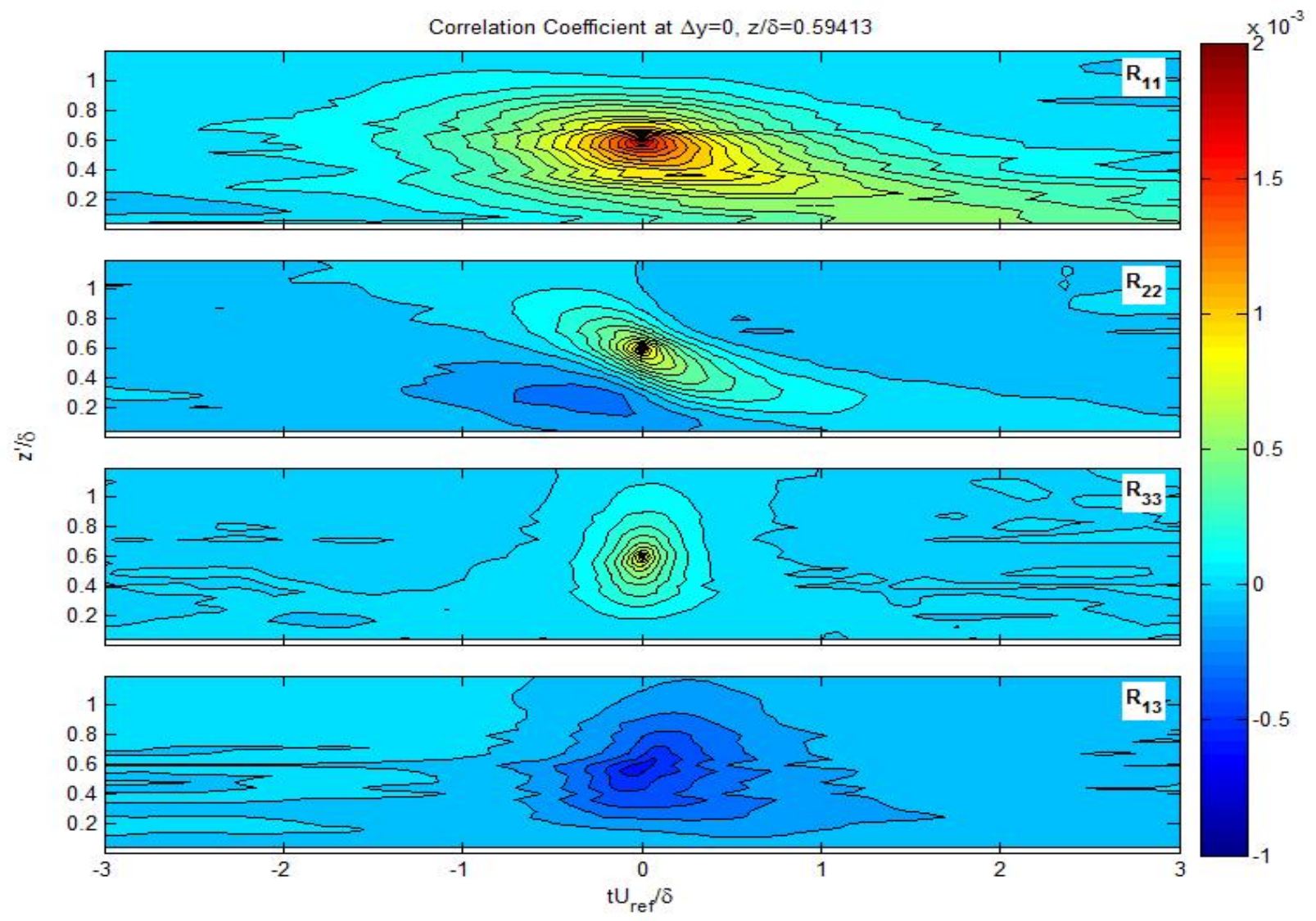

Video 1. Normal-to-wall velocity correlations at zero spanwise separation, $R_{i j}\left(0, z, z^{\prime}, \tau\right)$ 


\subsubsection{Spanwise Time Delay Correlation}

The final velocity correlation to be analyzed is the spanwise correlation as it evolves over time. The spanwise time delay correlation is found by evaluating the correlation tensor at various spanwise separations when both probes are at the same normal-to-wall distance $\left(R_{i j}\left(\Delta y, z=z^{\prime}, z^{\prime}, \tau\right)\right)$. A video representation of this can be seen in Video 2 where spanwise separation and normal-to-wall distance are normalized on boundary layer thickness and time is again normalized on boundary layer thickness over edge velocity to represent the streamwise separation. These correlation maps are symmetric about the $\Delta y=0$ line since the flow is homogeneous in the spanwise direction. The velocity fluctuations in the normal to wall direction $R_{33}$ exhibit nearly isotropic correlation throughout the boundary layer similar to that of the plane perpendicular to the wall. At probe locations close to the wall spanwise velocity fluctuations $R_{22}$ correlate over approximately 2 boundary layer thicknesses in the streamwise direction. Moving away from the wall $R_{22}$ quickly becomes nearly isotropic and extends over about 40 percent of the boundary layer. The streamwise velocity fluctuations $R_{11}$ are dissimilar from both $R_{22}$ and $R_{33}$. As spanwise separation increases the streamwise correlation decreases to zero at a location of 20 to 40 percent of the boundary layer thickness depending on the normal to wall position of the probes. Following this zero correlation point the correlation drops to a minimum around 50 to 70 percent of $\delta$, again depending upon the normal to wall probe location. In the streamwise direction the $R_{11}$ velocity fluctuations are correlated for approximately 4 boundary layer thicknesses close to the wall and 2 boundary layer thicknesses near the middle of the boundary layer. This elongated flow structure is of aeroacoustic importance since the blades of a rotor operating in the boundary layer are likely to cut it multiple times. Ignoring any distortion of the turbulence, the blades of a $0.46-\mathrm{m}$ diameter scaled version of Sevik's 10 -bladed rotor, operated at the design advance ratio of 1.17 , would cut this correlation function at intervals of $t U r e f / \delta=0.53$ thus creating the haystacking effect. 


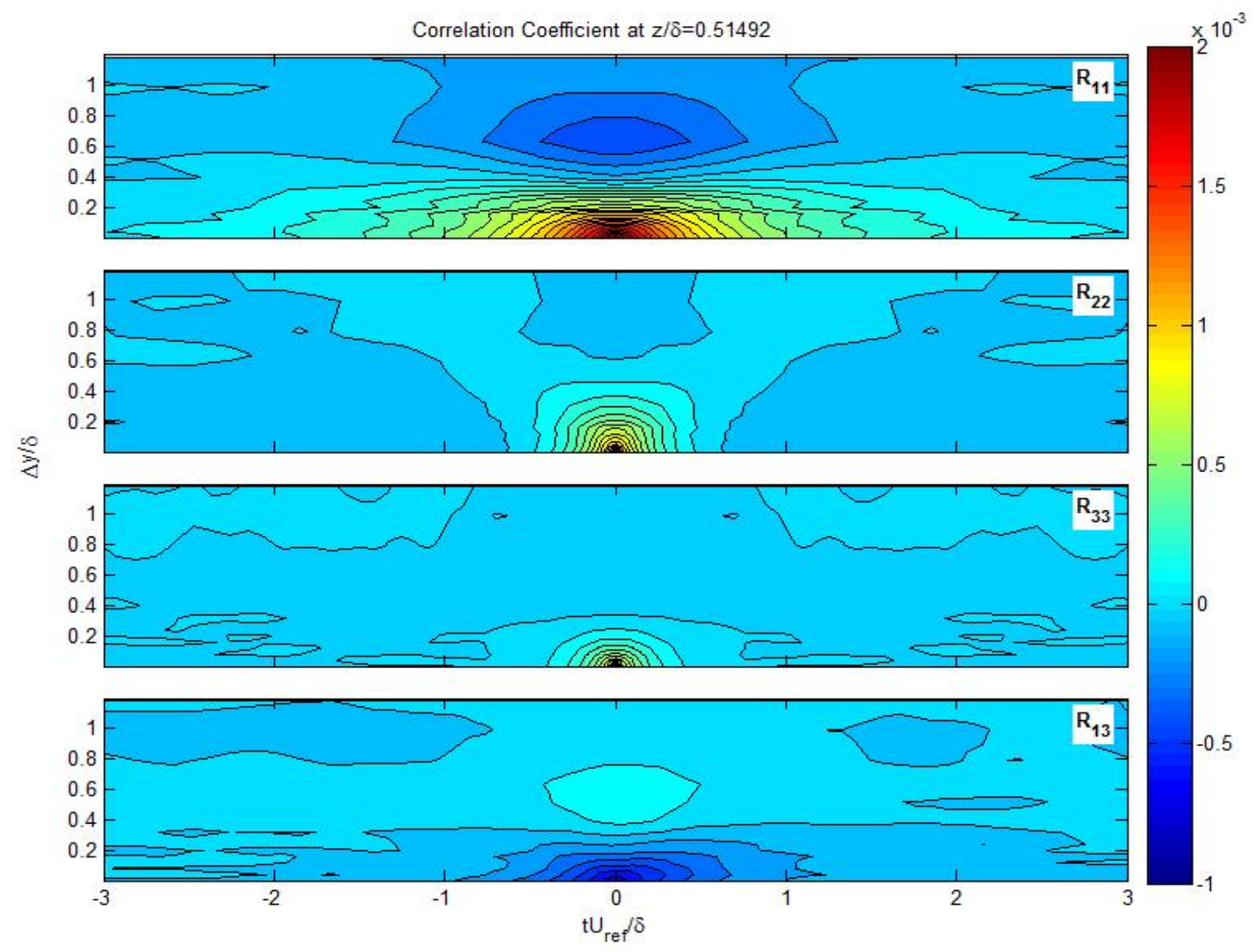

Video 2. Spanwise time delay correlation, $R_{i j}\left(\Delta y, z=z^{\prime}, z^{\prime}, \tau\right)$

\subsubsection{Comparison to DNS Channel Flow}

As discussed in Section 2.5, the two point measurement grids were designed based on DNS channel flow simulations performed by Moser et al (1999). Figure 3.14 and Figure 3.15 show the comparison between the correlation coefficient at zero time delay of the present study with that of Moser. The correlation coefficient of each data set has been normalized on the corresponding Reynolds stress, $R_{i j}\left(0, z, z^{\prime}=z, 0\right)$, to allow for an accurate comparison. At a fixed probe location of $z / \delta=20 \%$ the correlation coefficients are almost identical. Streamwise velocity fluctuations exhibit a nearly isotropic positive correlation around zero spanwise separation. Spanwise velocity fluctuations correlate over a wider range in the spanwise direction than in the normal to wall direction. $R_{22}$ and $R_{13}$ both correlate over a greater distance in the normal to wall direction than in the spanwise direction but only $R_{13}$ exhibits negative correlation lobes at larger spanwise separations. Differences in the correlation between studies occur toward the edge of the boundary layer, as one might expect given the difference in upper boundary condition. DNS channel flow simulations show that at a fixed probe location of $95 \%$ of the boundary layer thickness 
correlation coefficients $R_{11}$ and $R_{13}$ become directional and shift toward negative $y$ separation as the wall is approached illustrating inhomogeneous flow in the $y$ direction. The similarity between the two correlation functions throughout the majority of the boundary layer justifies the design of the two-point measurement grids.
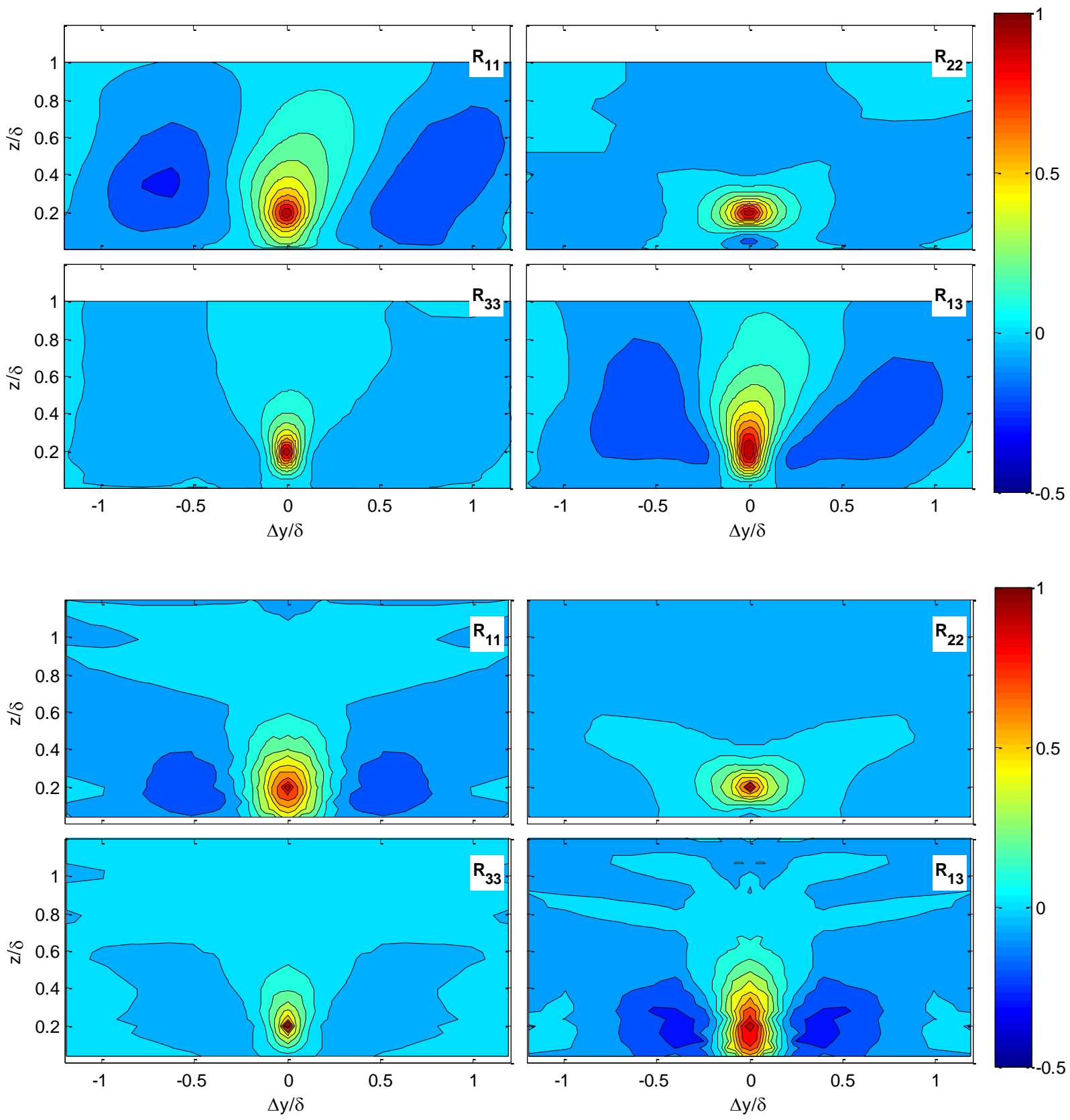

Figure 3.14. Comparison between the correlation function of Moser (top) and the present study (bottom) at a fixed probe location of $z / \delta=20 \%$ 

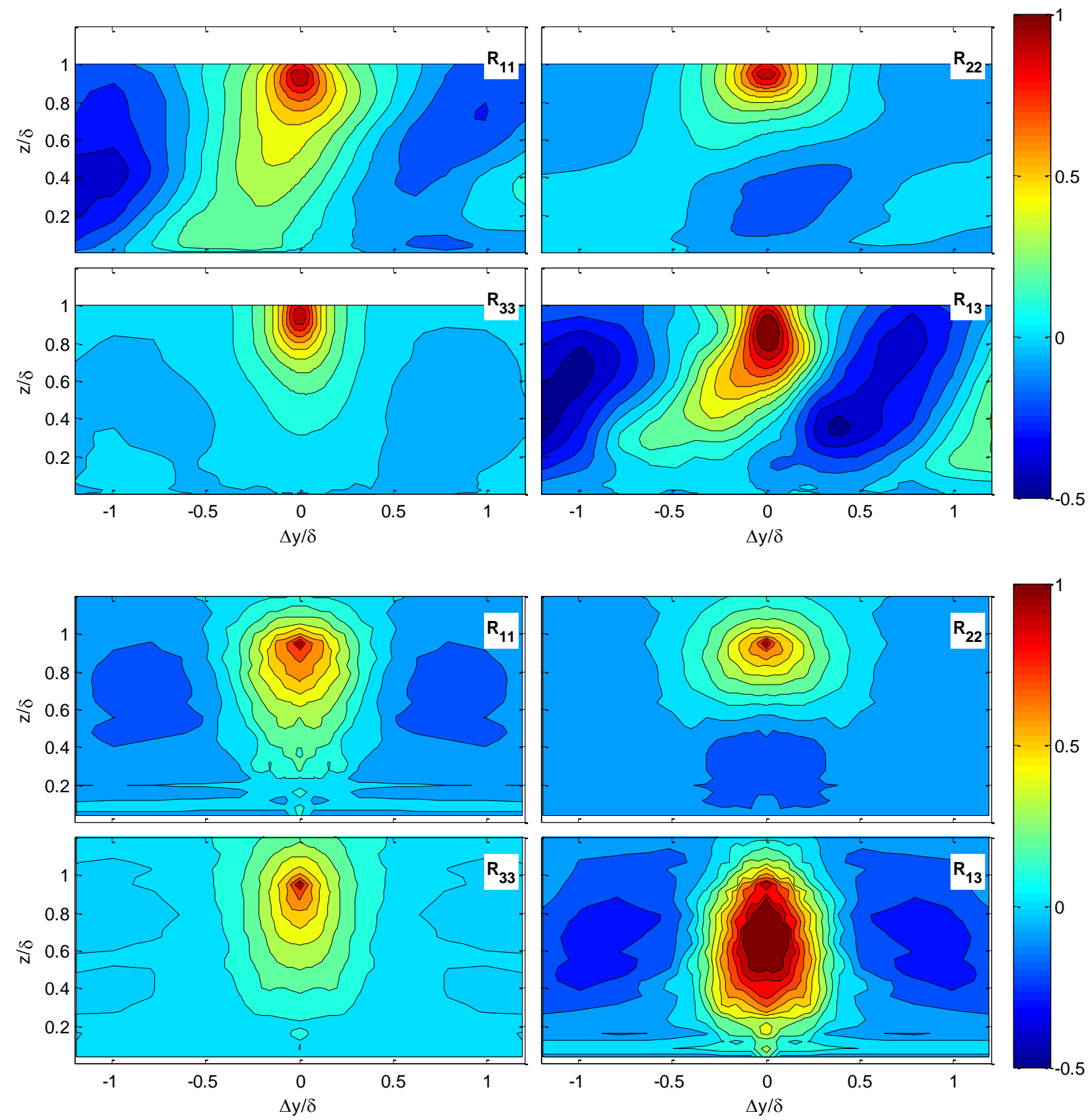

Figure 3.15. Comparison between the correlation function of Moser (top) and the present study (bottom) at a fixed probe location of $z / \delta=95 \%$

\subsubsection{Proper Orthogonal Decomposition and Linear Stochastic Estimation}

The correlation function discussed in the previous section is useful when describing the organization of the coherent structures. However, it is difficult to determine the form of the instantaneous eddy structures responsible for these correlation maps by only examining the velocity correlation. Therefore is becomes desirable to pursue a more objective and efficient method for extracting physical information about the instantaneous flow. Proper Orthogonal Decomposition (POD) and Linear Stochastic Estimation (LSE) are two methods that extract instantaneous velocity fields from the correlation function. 
Proper Orthogonal Decomposition is used to determine a series of orthogonal functions, or modes, that on average provide a best fit to the instantaneous velocity field. Lumley (1967) shows that these modes are eigenfunctions of the two-point correlation tensor and thus the proper orthogonal modes can be obtained by solving the Fredholm integral equation:

$$
\int R_{i j}\left(z, z^{\prime}, 0\right) \varphi_{j}^{(n)}\left(z^{\prime}\right) d z^{\prime}=\lambda^{(n)} \varphi_{i}^{(n)}(z)
$$

where $n$ is the mode number, $\varphi^{(n)}$ are the POD modes, and $\lambda^{(n)}$ are the eigenvalues. The eigenvalues $\lambda^{(n)}$ correspond to the proportion of the turbulence kinetic energy produced by each mode. This means that selecting the first few modes that comprise the majority of the total kinetic energy represents an accurate description of the flow.

In homogeneous directions POD reduces to Fourier decomposition which does not provide a good representation of eddies. Glegg and Devenport (2001) show that by using the POD modes that describe the flow in the inhomogeneous direction with Linear Stochastic Estimation (LSE) in the homogeneous direction, the full three-dimensional velocity field can be determined. LSE minimizes the error between the turbulent fluctuations and the linear estimate of the instantaneous velocity field. The linear estimate of the instantaneous velocity field at a point can be found from the velocity at another point and the correlation function between these two points by the equation:

$$
u_{j}^{L S E}\left(z^{\prime}\right)=A_{i j}(z) u_{i}(z)
$$

Equation 3.10

where $u_{j}^{L S E}$ is the linear estimate and $A_{i j}$ is the estimation coefficients. By choosing the estimation coefficients to minimize the mean square error between the predicted and measured velocity Equation 3.10 becomes

$$
\left.u_{j}^{L S E}\left(z^{\prime}, y^{\prime}-y, \tau\right)\right|_{L S E}=R_{i j}\left(z, z^{\prime}, y^{\prime}-y, \tau\right)\left[u_{i}(z) / \overline{u_{\imath}(z)^{2}}\right]
$$

and now the estimated velocity field, $\left.u_{j}\left(z^{\prime}, y^{\prime}-y, \tau\right)\right|_{L S E}$, is given by the two-point correlation function $R_{i j}\left(z, z^{\prime}, y^{\prime}-y, \tau\right)$.

The eigenvalue spectrum and cumulative energy spectrum can be seen in Figure 3.16 and Figure 3.17 respectively. The 3 component velocity measurements at 31 normalto-wall locations allow for 93 modes to be calculated. Only the first 20 modes are shown since these account for more than $75 \%$ of the total kinetic energy. The eigenvalues decay relatively rapidly with mode number such that the first two modes represent $31 \%$ of the total kinetic energy in the flow and only six modes are required to account for more than $50 \%$ of the cumulative energy. This implies that the turbulence structure in the boundary layer is relatively simple compared to wake measurements performed by Borgoltz (2007) who needed 9 modes to comprise the same amount of energy. 


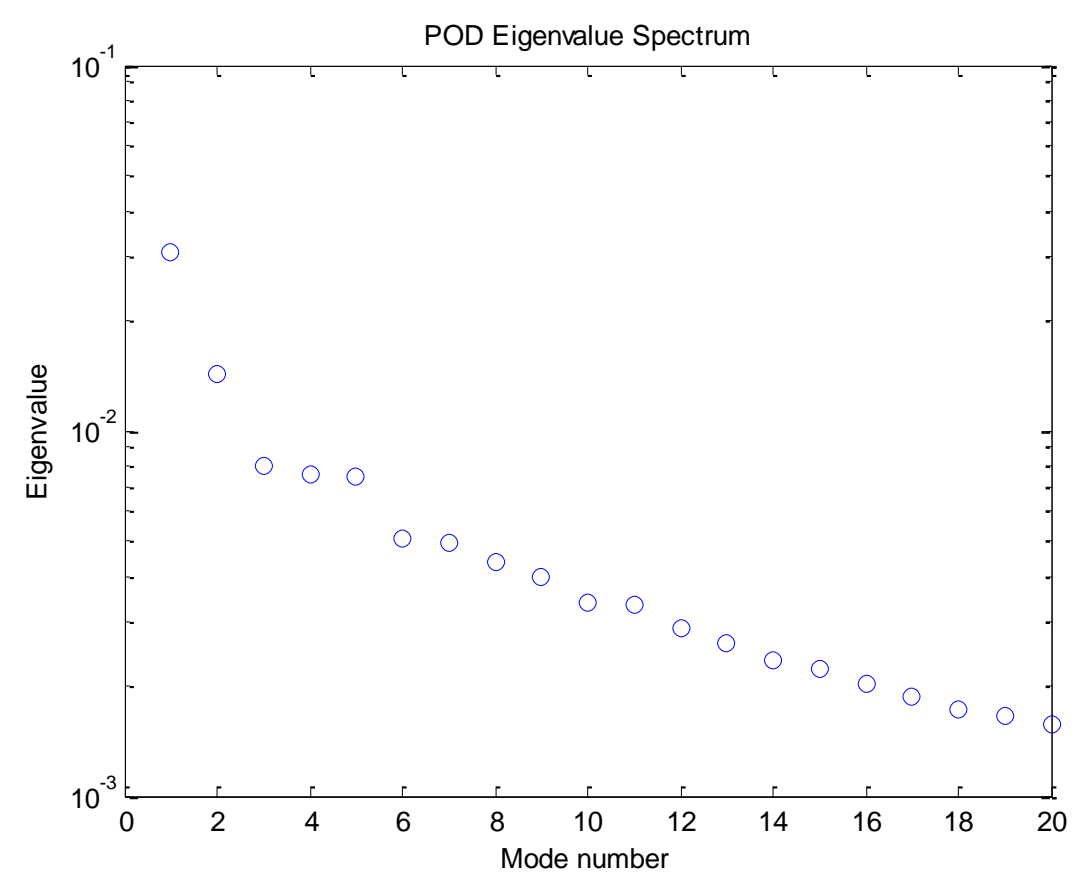

Figure 3.16. Eigenvalue spectrum for the first 20 modes

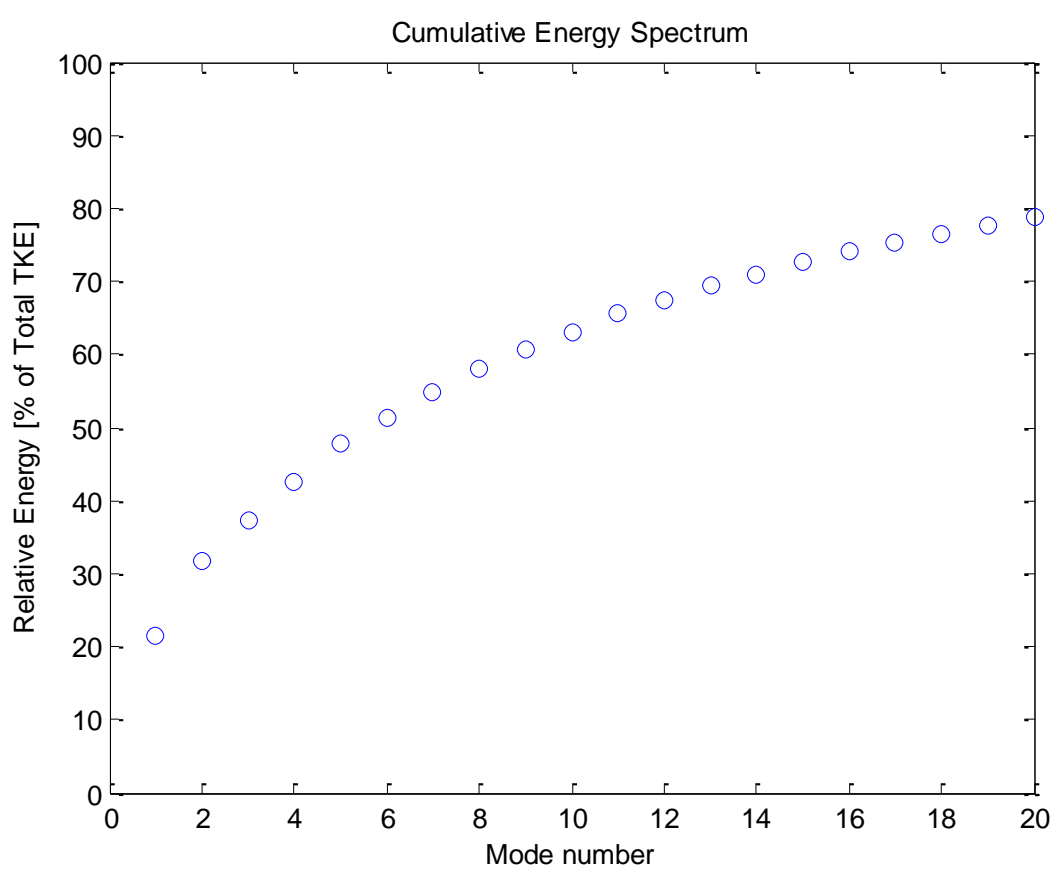

Figure 3.17. Cumulative energy spectrum for the first 20 modes

Shown in Figure 3.18 are the velocity profiles of the first four modes plotted against the normal-to-wall direction normalized on boundary layer. These velocity profiles are the eigenfunctions of the Fredholm integral equation thus they are normalized to have a mean square amplitude of one. The first mode, which is very energetic and contains 21 percent of the total kinetic energy, is dominated by the streamwise and normal-to-wall velocity components. The spanwise velocity component is negligible. Both profiles increase as the 
wall is approached until a maximum around $z / \delta=0.2$ then decrease. Mode 2 is also dominated by the streamwise and normal to wall velocity fluctuations. This modal shape demonstrates increasing normal-to-wall and streamwise velocity magnitudes as the wall is approached until $z / \delta=0.5$ then they decrease to zero and switch direction. This motion could represent multiple eddies in the $x z$-plane. Mode 3 is dominated by spanwise velocity fluctuations and demonstrates negligible normal-to-wall and streamwise velocities. Flow with this profile could be represented by a large structure stretched in the spanwise direction along the wall. Mode 4 is qualitatively similar to mode 2 except there are two locations where the streamwise and normal-to-wall velocities switch signs, $z / \delta=0.45$ and $z / \delta=0.15$. Again, this would represent flow containing structures rotating in the $x z$-plane.
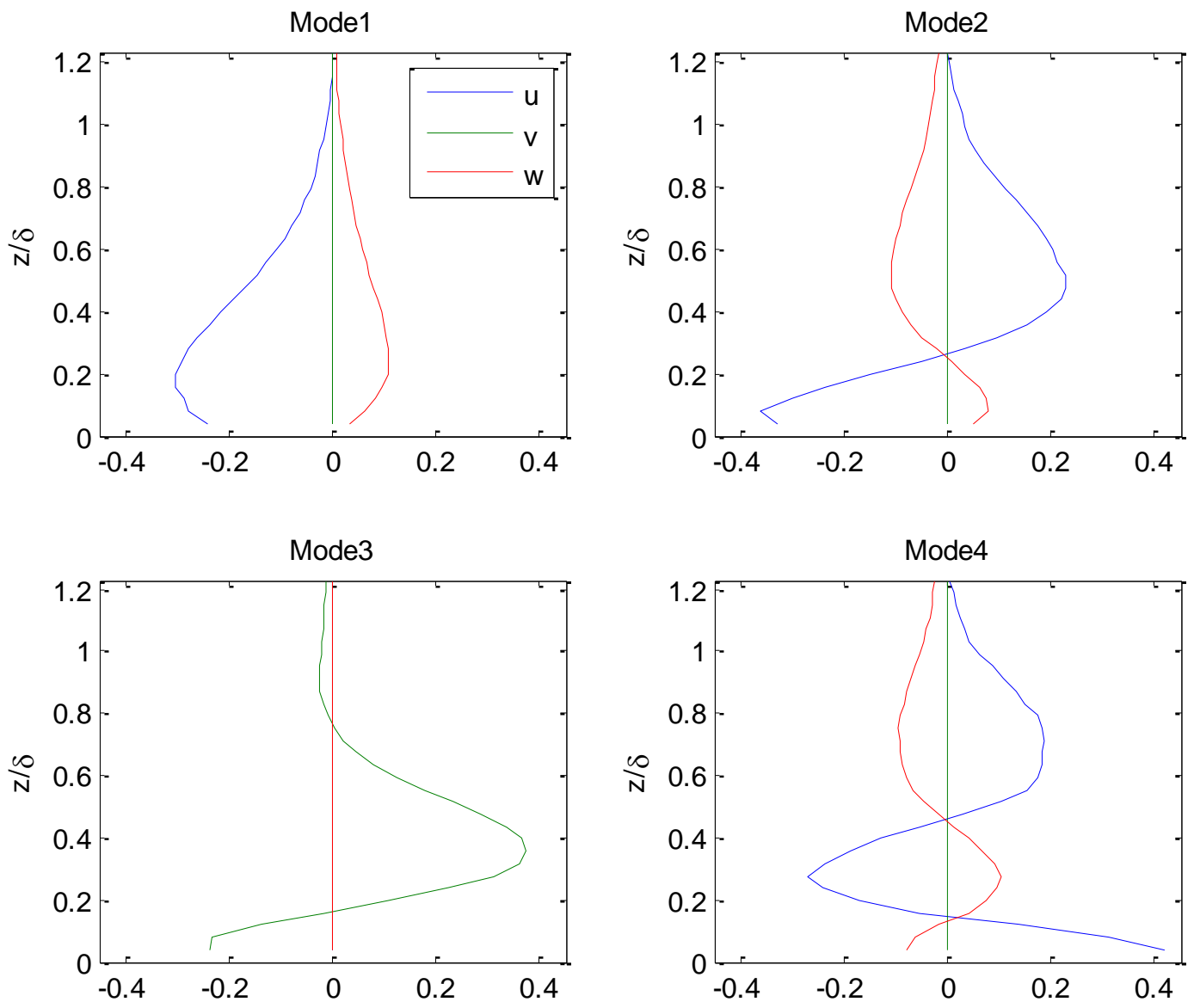

Figure 3.18. Modal Profiles of the first four modes determined from Proper Orthogonal Decomposition (POD).

\subsubsection{Compact Eddy Structures}

As discussed in the previous section, a statistically averaged view of the full threedimensional instantaneous velocity field can be determined by combining the POD modes in the inhomogeneous direction with Linear Stochastic Estimation in the homogenous direction. The resulting velocity fields are then given by: 


$$
\left.u_{j}^{(n)}\left(z^{\prime}, y^{\prime}-y, \tau\right)\right|_{C E S}=\frac{1}{\lambda^{(n)}} \int \varphi_{i}^{(n)}(z) R_{i j}\left(z, z^{\prime}, y^{\prime}-y, \tau\right) d z
$$

These three-dimensional modes are termed compact eddy structures (CES) (Glegg and Devenport, 2001) and are obtained by taking the inner product of the correlation function and the proper orthogonal mode. The compact eddy structures do not necessarily occur in the flow but rather they represent average structures. To fully visualize these three dimensional structures multiple cross sections are plotted in Figure 3.19 -Figure 3.21.

\subsubsection{Normal to Wall CES}

Cross sections at zero spanwise separation of the compact eddy structures for the first four modes of the flow are plotted in Figure 3.19. Each mode is normalized on its eigenvalue to show the structures present. In these plots a vector pointing up and to the right represents a positive streamwise and normal to wall velocity fluctuation. The first, and most energetic, mode demonstrates vectors stretched in the streamwise direction suggesting a large scale structure spanning close to $60 \%$ of the boundary layer. Mode 2 reveals a traditional small eddy structure centered around $z / \delta=25 \%$. Mode 3 is dominated by a negative spanwise flow close to the wall and a positive spanwise flow in the middle region of the boundary layer. This structure is also angled about 20 degrees from the wall suggesting a three dimensional structure. Mode 4 contains a small structure which strongly resembles the flow of mode 2 except the eddy is centered at $45 \%$ of the boundary layer height. These modes suggest the flow is arranged such that smaller turbulent structures are located near the wall and larger structures closer to the edge of the boundary layer. 

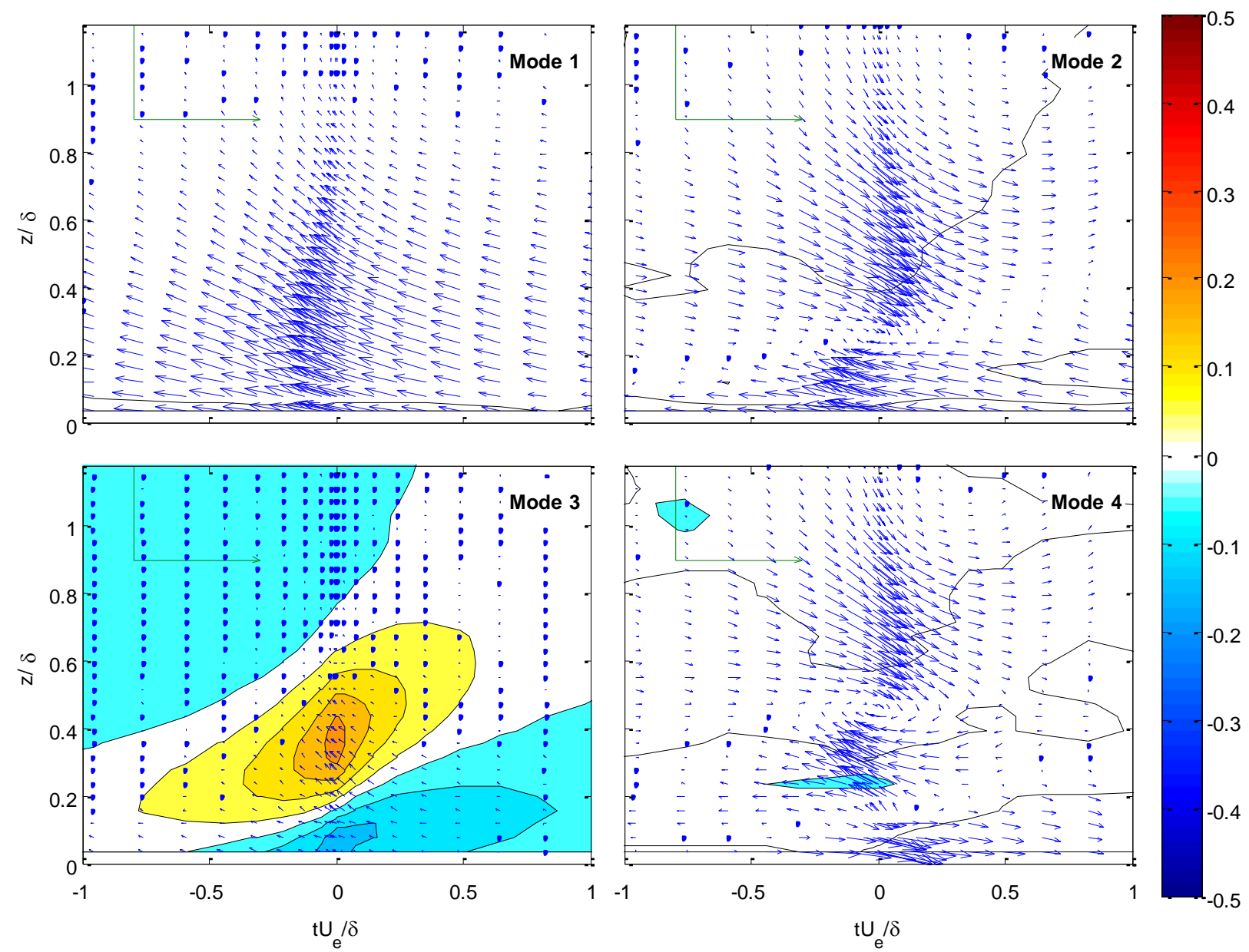

Figure 3.19. Compact Eddy Structures of the first four modes with eigenvalue normalization

\subsubsection{Spanwise CES}

Cross sections at various normal to wall distances allow the full three dimensionality of the flow to be seen. Figure 3.20 and Figure 3.21 show the first 4 modes at normal to wall distances of $8 \%, 24 \%, 55 \%$, and $102 \%$ of the boundary layer thickness. The flow does not exhibit anything of importance at locations greater than $55 \%$. The first mode shows a very similar structure throughout $55 \%$ of the boundary layer suggesting a large three dimensional structure. Modes 2 and 4 exhibit similar structures in the outer portion of the boundary layer but counter rotating structures close to the wall. The second mode seems to have one dominant structure in the boundary layer whereas the cuts through the boundary layer of mode 4 suggest 2 separate eddies. Mode 3 has an inclined structure relative to the wall which can be seen by the first three cuts through the boundary layer. The velocity fluctuations further from the wall are stronger at the non-dimensionalized time of 0 than they are at the near wall location which also exhibits velocity fluctuations in the opposite direction. 

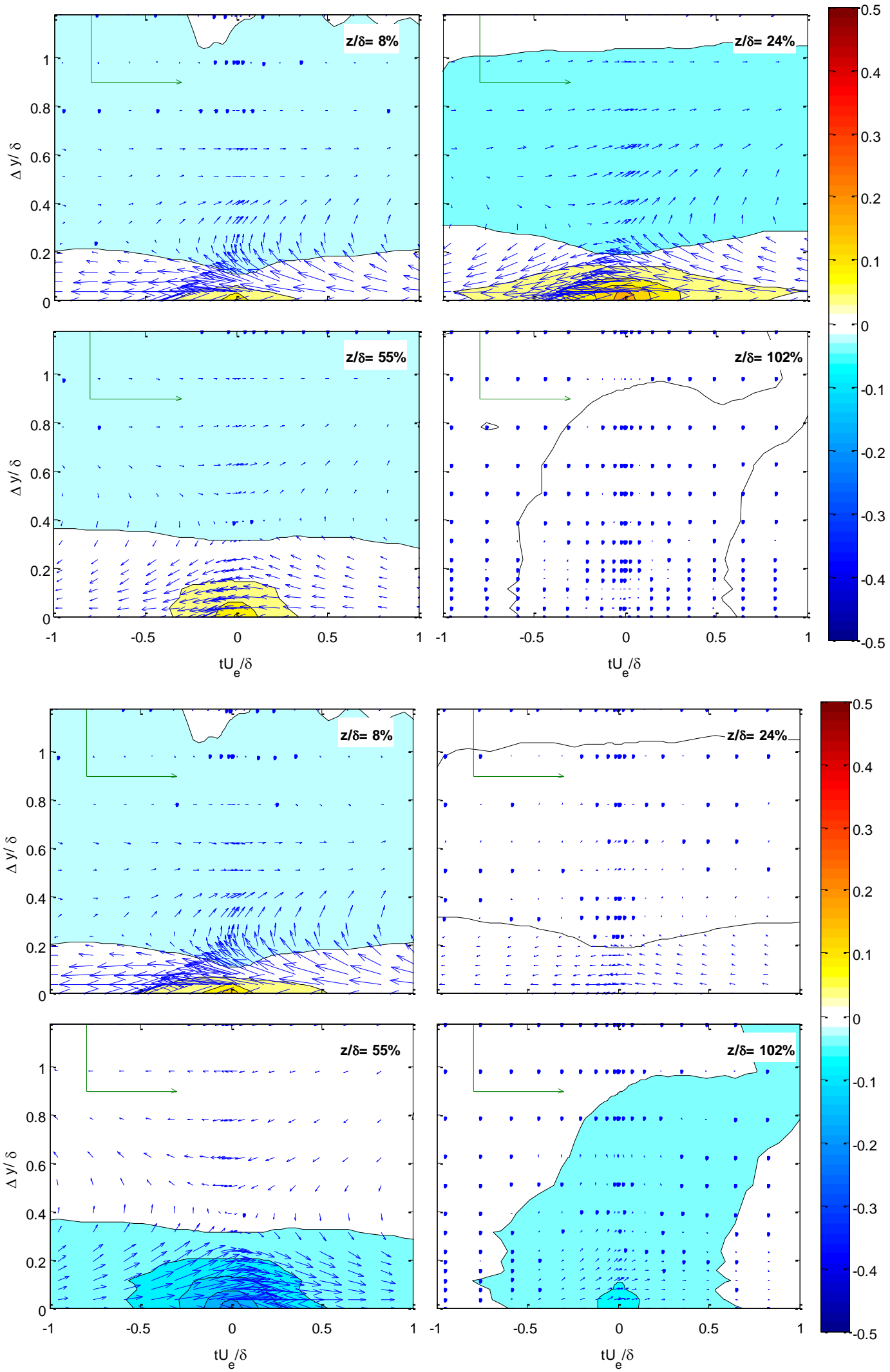

Figure 3.20. Compact Eddy Structures for mode 1 (top) and mode 2 (bottom) at various normal to wall locations 

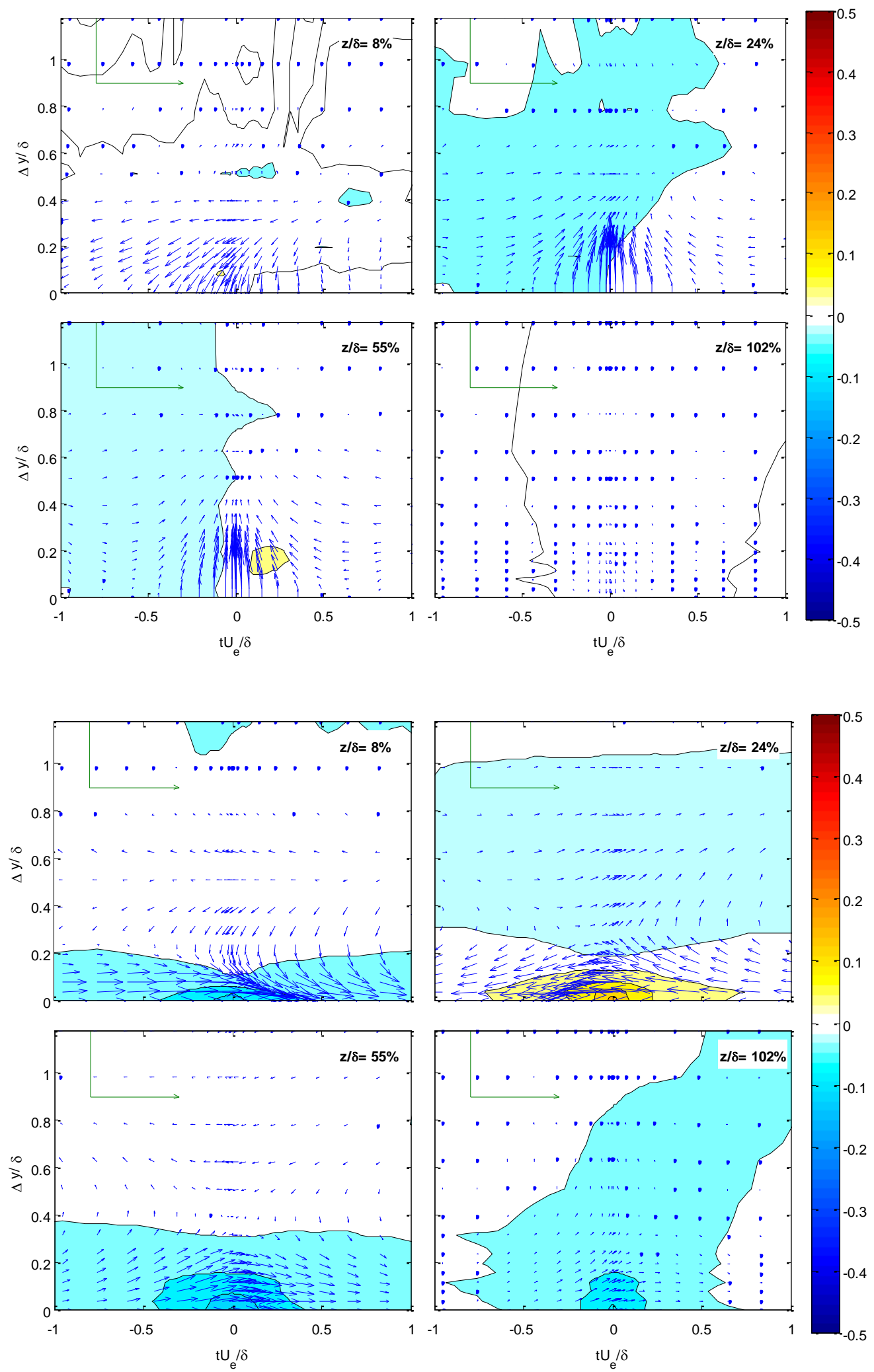

Figure 3.21. Compact Eddy Structures for mode 3 (top) and mode 4 (bottom) at various normal to wall locations 


\subsection{Sounds Predictions}

\subsubsection{Sound Prediction Methodology}

A theory for the sound radiated from unsteady loading on a rotor due to blades cutting inhomogeneous turbulence has been developed by Glegg et al (2012). The solution to Lighthill's wave equation given by Ffowcs Williams and Hawkings gives the acoustic field from a blade with a net blade surface loading per unit area of $f_{i}(z, \tau)$ as shown in Equation 3.13 .

$$
p^{\prime}(\boldsymbol{x}, t)=-\frac{\partial}{\partial x_{i}} \sum_{n=1}^{B} \int_{\Sigma n}\left[\frac{f_{i}(\mathbf{z}, \tau)}{4 \pi r\left|1-M_{r}\right|}\right] d \sum(\mathbf{z})
$$

where $\boldsymbol{x}$ is the observer location, $\sum_{n}$ is the planform of each blade, $B$ the number of blades, and $r$ the distance from the source to observer. Assuming compact chord and using a strip theory approach to relate the blade upwash to unsteady lift on the blade a theory is developed for the far-field sound pressure spectrum $S_{p p}$, the details of which can be found in Glegg et al. (2012). The far-field sound spectrum is given as:

$$
\begin{aligned}
S_{p p}(\boldsymbol{x}, \omega)= & \left(\frac{\omega}{c_{o} r_{o}}\right)^{2} \sum_{n=1}^{B} \sum_{k=1}^{B} \sum_{m=-\infty}^{\infty} \\
& \int_{R_{\text {hub }}}^{R_{\text {tip }}} \int_{R_{\text {hub }}}^{R_{\text {tip }}} K_{m}(R, \boldsymbol{x}, \omega) K_{m}^{*}\left(R^{\prime}, \boldsymbol{x}, \omega\right) S_{L L}^{(n, k)}\left(R, R^{\prime}, \omega-m \Omega\right) e^{-m\left(\varphi_{n}-\varphi_{k}\right)} d R d R^{\prime}
\end{aligned}
$$

Equation 3.14

where $\omega$ is the angular frequency, $c_{0}$ the speed of sound, $\Omega$ the angular velocity of the rotor, and $\varphi_{n}$ the angle to the leading edge of the $n^{\text {th }}$ blade in the plane of the rotor. The coordinate system used for sound predictions can be seen in Figure 3.22. The double integration over the radial length of each blade takes into account the radial extent of the lift correlation while the double summation over the $n^{\text {th }}$ and $k^{\text {th }}$ blades considers the blade to blade correlation of the unsteady lift spectrum. The $K_{m}$ terms are Fourier coefficients that determine the propagation of sound from the source on the blade to the observer at location $\boldsymbol{x}$ and take into account the retarded time effects due to the rotation of the blades. The Fourier series coefficients are found from the expansion of the periodic function in brackets in Equation 3.15:

$$
-\frac{x_{i} n_{i}(R, \tau) e^{\frac{i \omega r(\tau)}{c_{o}}}}{4 \pi r(\tau)}=\sum_{m=-\infty}^{m=\infty} K_{m}(R, x, \omega) e^{-i m(\Omega \tau+\varphi)}
$$

where $n_{i}$ are the components of the unit normal of the blade at radial distance $R$ and source time $\tau$. The unsteady loading spectrum $S_{L L}$ in Equation 3.14 is determined from the upwash spectrum $S_{w w}$ on the blades by a straightforward strip-theory approach: 


$$
S_{L L}^{(n, k)}\left(\omega, R, R^{\prime}\right)=\left[\pi \rho_{o} c U_{e} S(\sigma)\right]_{R}\left[\pi \rho_{o} c U_{e} S(\sigma)\right]_{R}^{*} S_{w w}^{(n, k)}
$$

where $\rho_{0}$ is the flow density, $U_{e}$ is the effective velocity, and $S(\sigma)$ is the two-dimensional Sears function given by:

$$
S=\frac{2}{\pi \sigma\left(H_{0}^{(1)}(\sigma)+H_{1}^{(1)}(\sigma)\right)} \quad \sigma=\omega c / 2 U_{e}
$$

If $\mathbf{Z}^{(n)}(\tau)$ is taken as the location in hub fixed coordinates of the $n^{\text {th }}$ blade at source time $\tau$, the unsteady upwash cross-spectrum can be written as:

$$
S_{w w}^{(n, k)}\left(\omega, R, R^{\prime}\right)=\frac{1}{4 \pi^{2}} \frac{\pi}{T} \int_{-T}^{T} \int_{-T}^{T} n_{i}^{(n)}(R, \tau) n_{j}^{(k)}\left(R^{\prime}, \tau^{\prime}\right) R_{i j}\left(\mathbf{z}_{n}(\tau), \mathbf{z}_{\boldsymbol{k}}\left(\tau^{\prime}\right), \tau-\tau^{\prime}\right) e^{i \omega\left(\tau-\tau^{\prime}\right)} d \tau d \tau^{\prime}
$$

Equation 3.18

where $T$ is taken sufficiently large such that $\Omega T \gg>1$, and $R_{i j}$ is the two-point space time correlation function in the boundary layer. This is the exact function discussed in Section 3.4.2 but evaluated in a blade frame of reference coordinate system.

This theory predicts the sound radiation from an open rotor and neglects the effect of the wall on the sound propagation. To include this effect, since it will be necessary to compare to future measurements, the Fourier series coefficients in Equation 3.15 have been modified and can be calculated from the periodic function in brackets given by:

$$
\begin{array}{r}
\left\{\frac{\delta}{\delta x_{i}}\left(\frac{n_{i}(R, \tau) e^{\frac{i \omega r(\tau)}{c_{o}}}}{4 \pi r(\tau)}\right)+\frac{\delta}{\delta x_{i}^{\#}}\left(\frac{n_{i}(R, \tau) e^{\frac{i \omega r^{\#}(\tau)}{c_{o}}}}{4 \pi r(\tau)}\right)\right\} \\
=\frac{i \omega}{c_{o} r_{o}} \sum_{m=-\infty}^{m=\infty} K_{m}(R, x, \omega) e^{-i m(\Omega \tau+\varphi)}
\end{array}
$$

where $r^{\#}$ and $x_{i}^{\#}$ are evaluated at $\left(x_{1}, x_{2}, x_{3}-2 h\right)$. Since the sound radiated from the noise source is of particular interest and not necessarily the effects of the wall on sound propagation, the sound field generated neglecting the wall will be analyzed in detail in Section 3.5.5.1 followed by a discussion of the wall effects in Section 3.5.5.2. 


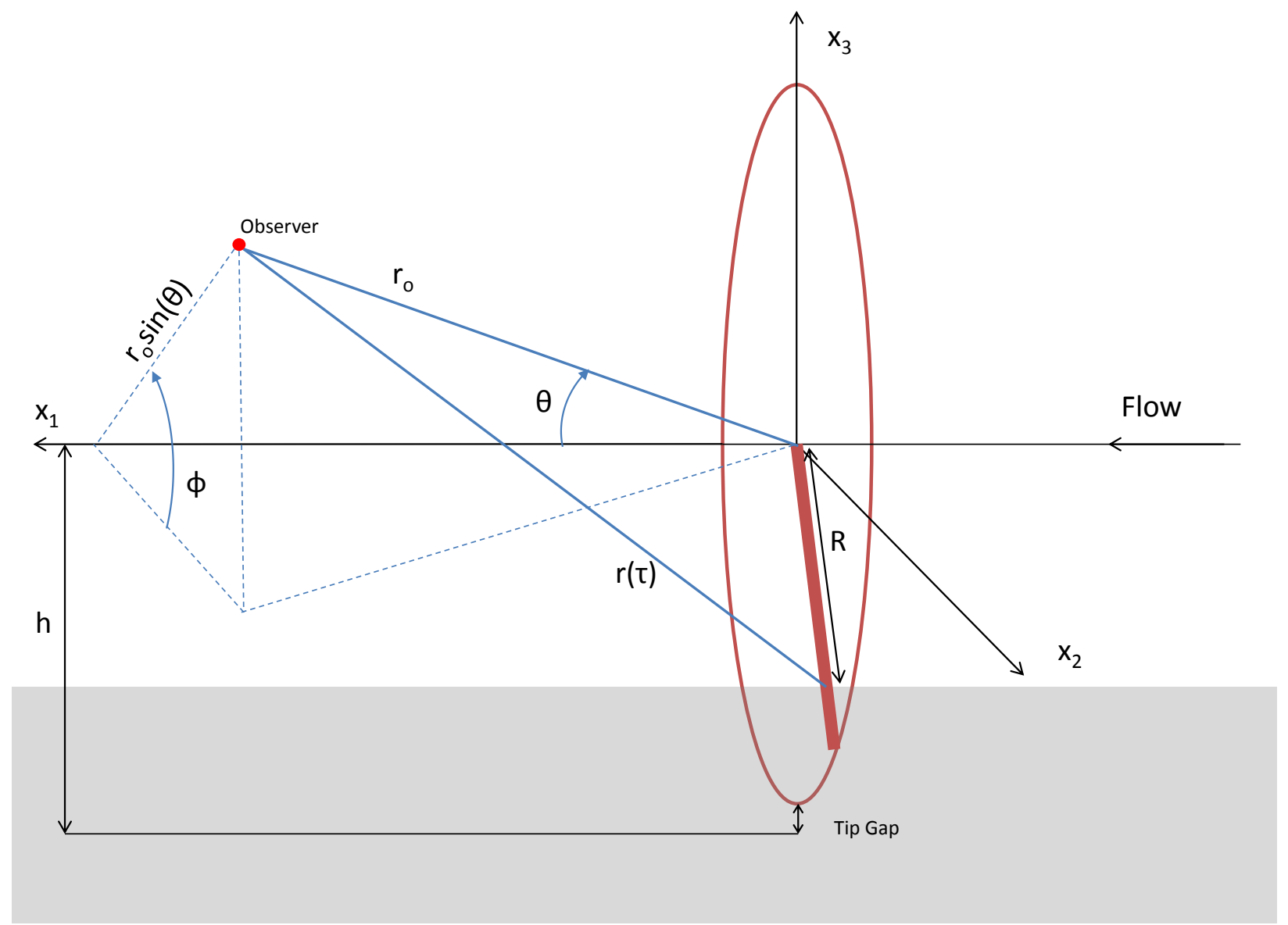

Figure 3.22. Coordinate system used for sound predictions

\subsubsection{Rotor Geometry}

The rotor geometry used for the design sound predictions is a 2.25 scaled version of the Sevik (1970) rotor as this geometry is actually being built at Virginia Tech for future testing. The rotor consists of ten equally spaced blades each having a constant chord of $57.2 \mathrm{~mm}$ along their span, no skew and square tips. A schematic of the rotor as well as profiles of the blade at its tip and root can be seen in Figure 3.23. The outer diameter of the rotor measures $457.2 \mathrm{~mm}$ while the hub diameter has been increased in size beyond the 2.25 scaling to $127 \mathrm{~mm}$ to provide room for instrumentation within the hub. To account for this increase in hub diameter, the blades were shortened by $6.4 \mathrm{~mm}$ at their root causing each blade to have a span of $165.1 \mathrm{~mm}$. The blade pitch angle measured from the plane of the rotor varies nonlinearly from the root to the tip as shown in Table 2. 

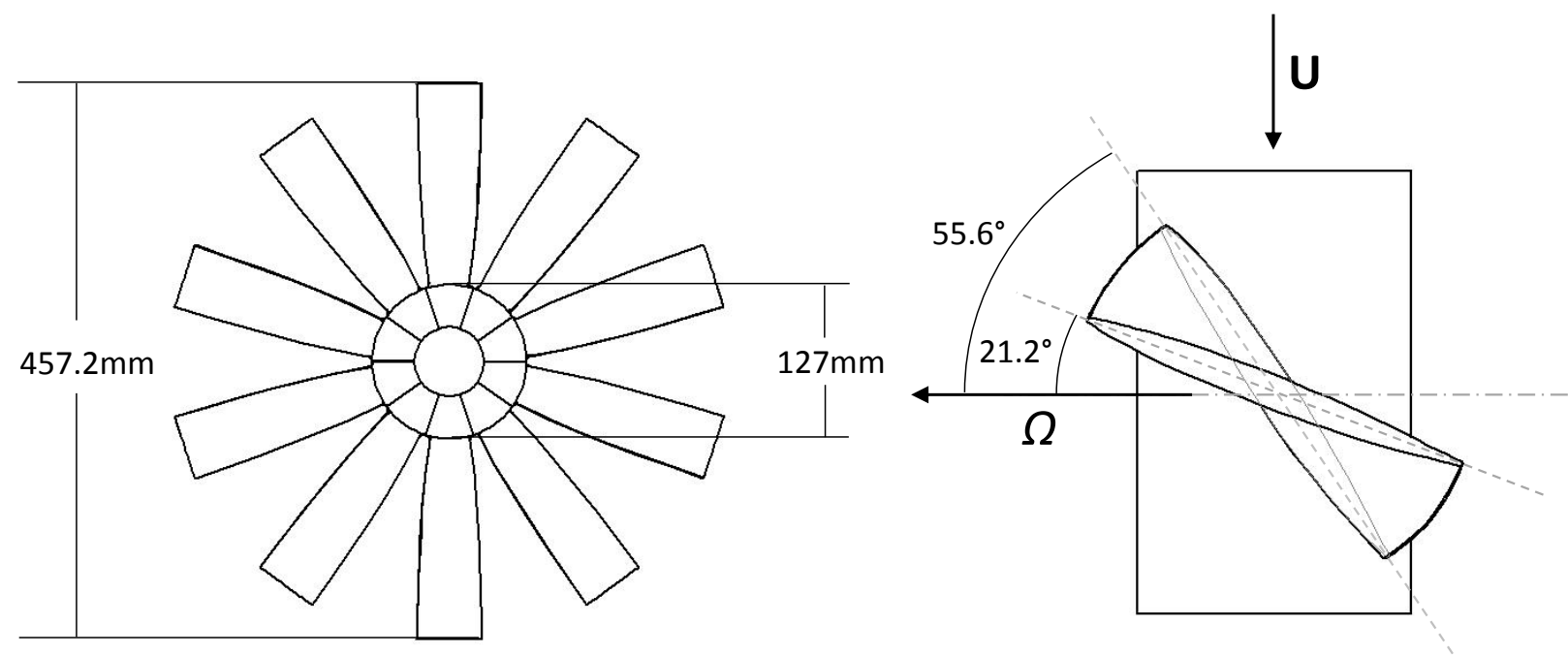

Figure 3.23. Schematic of the rotor with profiles of the blades at the tip and root

\begin{tabular}{|c|c|}
\hline $\mathrm{r} / \mathrm{R}$ & $\begin{array}{c}\text { Blade Angle } \\
\text { (degrees) }\end{array}$ \\
\hline 0.28 & 55.60 \\
\hline 0.34 & 52.62 \\
\hline 0.40 & 47.78 \\
\hline 0.46 & 43.56 \\
\hline 0.52 & 39.87 \\
\hline 0.58 & 36.60 \\
\hline 0.64 & 33.64 \\
\hline 0.70 & 30.94 \\
\hline 0.76 & 28.50 \\
\hline 0.82 & 26.30 \\
\hline 0.88 & 24.36 \\
\hline 0.94 & 22.66 \\
\hline 1.00 & 21.19 \\
\hline
\end{tabular}

Table 2. Blade angles along the radial distance of each blade measured from the plane of the rotor

The rotor operates at a design advance ratio of 1.17. Most of the analysis below has been performed at a baseline condition defined as operating at an advance ratio of 1.17 and a free stream flow velocity of $20 \mathrm{~m} / \mathrm{s}$. Also, in this case, the rotor axis is located a distance of $271.4 \mathrm{~mm}$ from the wall in a $101 \mathrm{~mm}$ boundary layer which creates a blade immersion depth of $80.7 \mathrm{~mm}$ and a tip gap of $20.3 \mathrm{~mm}$. At $20 \mathrm{~m} / \mathrm{s}$ the correlation was assumed to scale with the free stream velocity as justified by the scaling of the Reynolds stresses seen in Figure 3.8a.

\subsubsection{Source Terms as Seen in the Blade Reference Frame}

To analyze the correlation function in Equation 3.18 in a blade frame of reference coordinate system, two complete rotor rotations were discretized into 1024 source time intervals and blade radius was discretized into 8 values between $58 \%$ and $100 \%$ of the blade radius. Radial locations below $58 \%$ of the blade radius never travel into the boundary 
layer and are therefore not considered. In Equation 3.18 the second component of $\boldsymbol{z}_{k}\left(\tau^{\prime}\right)-$ $\boldsymbol{z}_{n}(\tau)$ is the spanwise separation $\Delta y$ and the third components of $\boldsymbol{z}_{n}(\tau)$ and $\boldsymbol{z}_{k}\left(\tau^{\prime}\right)$ are $z$ and $z^{\prime}$ respectively.

For a rotor with evenly spaced identical blades, the absolute indices $n$ and $k$ are not important but rather the difference in blade indices defined as $p=n-k$. Video 3 shows the streamwise velocity correlation $R_{11}$ seen by the rotor blade tips at various blade separations. Only the streamwise component of the velocity correlation is shown here since it is approximately equal to the upwash seen on the blades. The vertical axis represents the normalized time delay for the $n^{\text {th }}$ blade and the horizontal axis represents the normalized time delay for the $k^{\text {th }}$ blade both spanning two rotor rotations. A time coordinate of zero represents a blade location at top dead center as far out as the boundary layer as possible. At zero blade separation $(p=0)$, the diagonal from the bottom left to top right represents the mean square of the turbulent velocity fluctuations at the blade tips. Following the diagonal the rotor blade tip interacts with significant turbulence between a $\tau \delta / U_{\text {ref }}$ of 1.9 and 3.3 as well as between a $\tau \delta / U_{\text {ref }}$ of 7.2 and 8.6 which corresponds to just more than $1 / 4^{\text {th }}$ of a rotor rotation. Moving away from the diagonal of each correlation island there is a decay representing the time delay correlation along the blade tip path. In the upper left and lower right section of the plot there are two echoes of the main correlations present. These are correlations between the first pass of the blade tip with the second, a direct consequence of the large size of the boundary layer compared to the rotor and the long streamwise scales of the turbulence. Increasing the blade separation to $p=1$ (adjacent blades) causes the two main correlation islands to shift to the right a distance equivalent to $1 / 10^{\text {th }}$ of a rotor rotation since this is a ten bladed rotor. These correlation islands exhibit a small decrease in magnitude because of the natural decay of the turbulence over this time. Also, the bottom right echo has vanished since now the correlation is so low between the first passing of the $n^{\text {th }}$ blade and the second passing of the $k^{\text {th }}$ blade. The opposite occurs in the upper left echo since now the first passing of the $k^{t h}$ blade with the second passing of the $n^{\text {th }}$ blade is only separated by 9/10ths of a rotor rotation. This simply represents the time delay between the leading and trailing blades cutting the same turbulence structures. This same trend occurs until a blade separation of $\mathrm{p}=5$. Here blade $\mathrm{n}$ and blade $\mathrm{k}$ are 180 degrees from each other; therefore both blades see the same correlation at each pass through the boundary layer. Increasing the blade separation further causes the correlation between the second pass of the $k^{\text {th }}$ blade with the first pass of the $n^{\text {th }}$ blade to become the dominant echo as expected. 


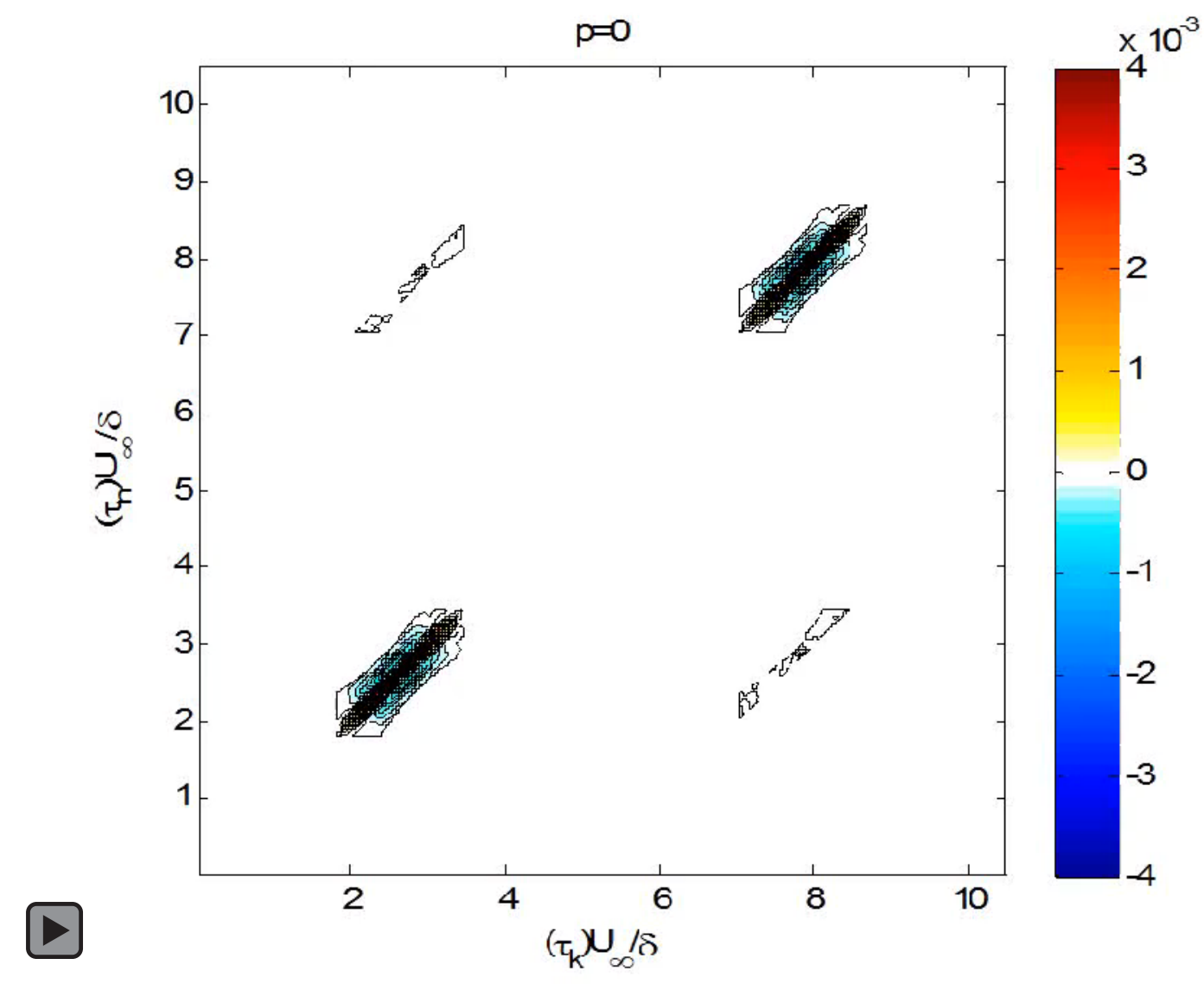

Video 3. Streamwise velocity correlation $R_{11}$ seen by the rotor blade tips at various blade separations.

\subsubsection{Upwash Coherence}

The unsteady upwash cross spectrum $S_{w w}$ in Equation 3.18 can be seen plotted in terms of coherence in Figure 3.24 Figure 3.28 and is exactly what will be measured in the future parts of this study. In Figure 3.24 the upwash coherence is evaluated between the blade tip and various radial locations of the same blade. There is a clear peak around a nondimensional frequency of 10 for each radial separation. By taking the timescale corresponding to this frequency and multiplying it by the blade tip speed, the corresponding spatial scale is found to be about 1.7 boundary layer thicknesses. Inferring the corresponding spatial scale we take the timescale corresponding to this frequency and multiply by the blade tip speed to get a value of 1.7 boundary layer thicknesses. This is evident in Video 3 where the streamwise length of the lobes of the $R_{11}$ correlation coefficient (the dominant component of the upwash) again corresponds to the same nondimensional frequency of 10 . There is significant coherence over 80 percent of the blade which corresponds to just fewer than half the thickness of the boundary layer. 


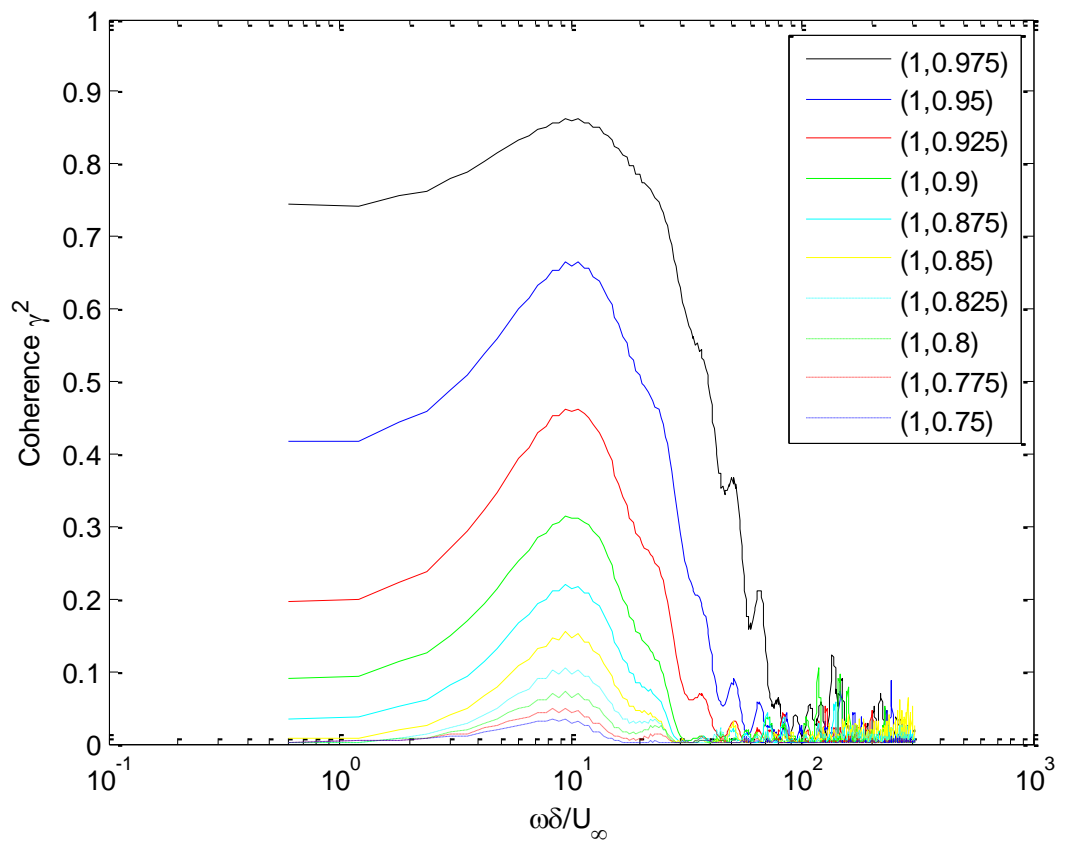

Figure 3.24. Spanwise coherence between velocity fluctuations at the blade tip and various radial locations on the same blade. $(R n=1, p=0)$

Figure 3.25 shows the coherence between velocity fluctuations at the blade tips for various blade separations. Here for a blade separation of $p=0$ the coherence is obviously unity. Maximum coherence for a blade separation other than zero occurs on adjacent blades ( $p=1)$ around the same non-dimensional frequency of 10 and has a value of about 0.44 . The maximum coherence values for a blade separation of $p=2$ drops significantly to about 0.2 . The coherence values for blade separations of $p=3$, 4, and 5 are all less than 0.1 . Rotational symmetry of the blades causes the coherence for blade separations of $p=6,7,8$, and 9 to be identical to $p=4,3,2$, and 1 respectively. This is due to the fact that, for example, the relationships between the velocity fluctuations between blades 1 and 2 are identical to those seen by blades 10 and 1 . The "saw-tooth" effect seen at lower frequencies is due to the unequal spacing in source time intervals, mainly the large spacing at larger time delays. 


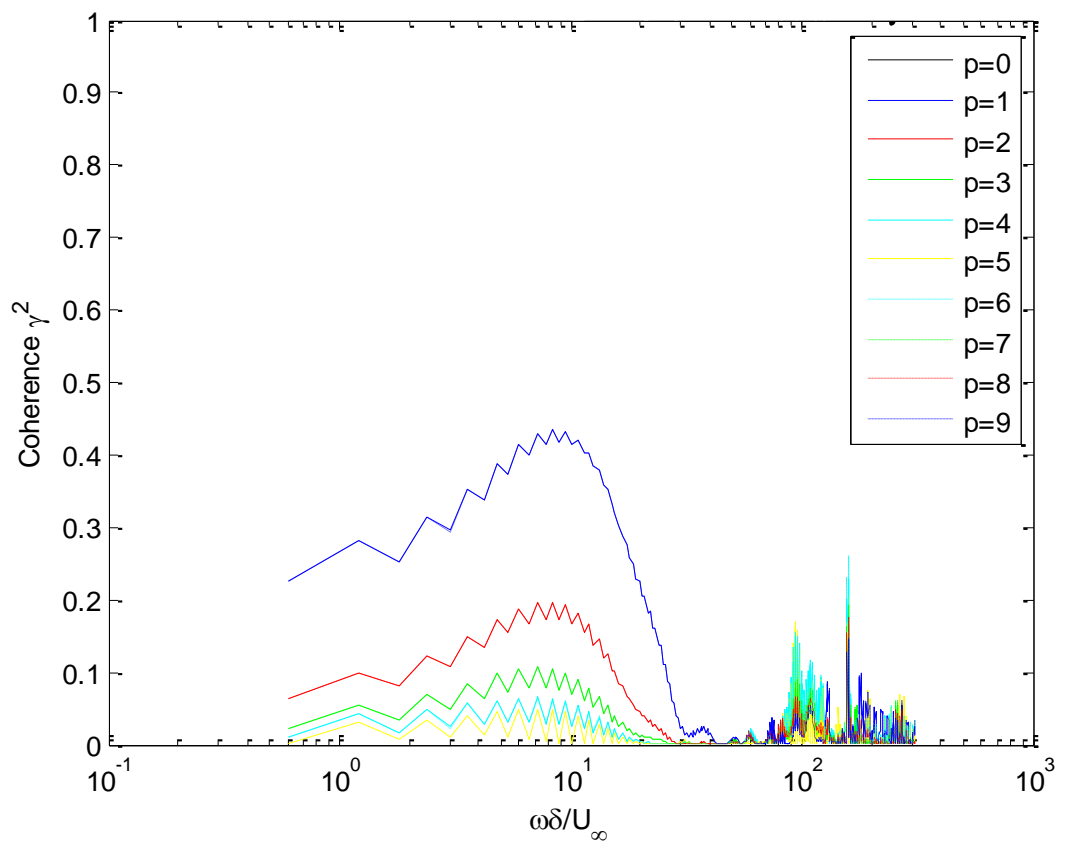

Figure 3.25. Blade to blade coherence between velocity fluctuations seen at the blade tip for different blade number differences $p .\left(R_{n}=R_{k}=1\right)$

The effect of the inclination of the boundary layer structures relative to the wall in the normal to wall correlation function can be seen in the coherence plots in Figure 3.26. This figure shows the blade to blade coherence between velocity fluctuations at the blade tip and at the $95 \%$ radial location. As blade separation increases from $p=1$ to $p=5$ coherence values decrease and are slightly less than the coherence values in Figure 3.25. The asymmetry is then present in the coherence values of blade separations greater than 5 . The dashed blue line represents a blade separation of $p=9$ and is no longer the same as a blade separation of $p=1$, in fact, it is significantly higher. This coherence at $p=9$ achieves a maximum of about 0.45 which is slightly higher than the maximum coherence between blade tips shown in Figure 3.25. Similarly, coherence values for blade separations of $p=6,7$, and 8 are higher than $p=4,3$, and 2 , respectively. The inclination of the boundary layer structures relative to the wall produce a correlation between velocity fluctuations in the upper part of the boundary layer and fluctuations seen at a later time close to the wall. Coherence between velocity fluctuations seen at the blade tip and $90 \%$ radial location exhibit similar characteristics with the coherence at zero blade separation and a blade separation of $p=9$ almost identical as show in Figure 3.27. As the distance between the tip and the radial location is increased the asymmetry causes the maximum coherence to occur at a blade separation of $p=9$ instead of $p=0$. Figure 3.28 shows an example of this at the radial location of $85 \%$ blade radius. 


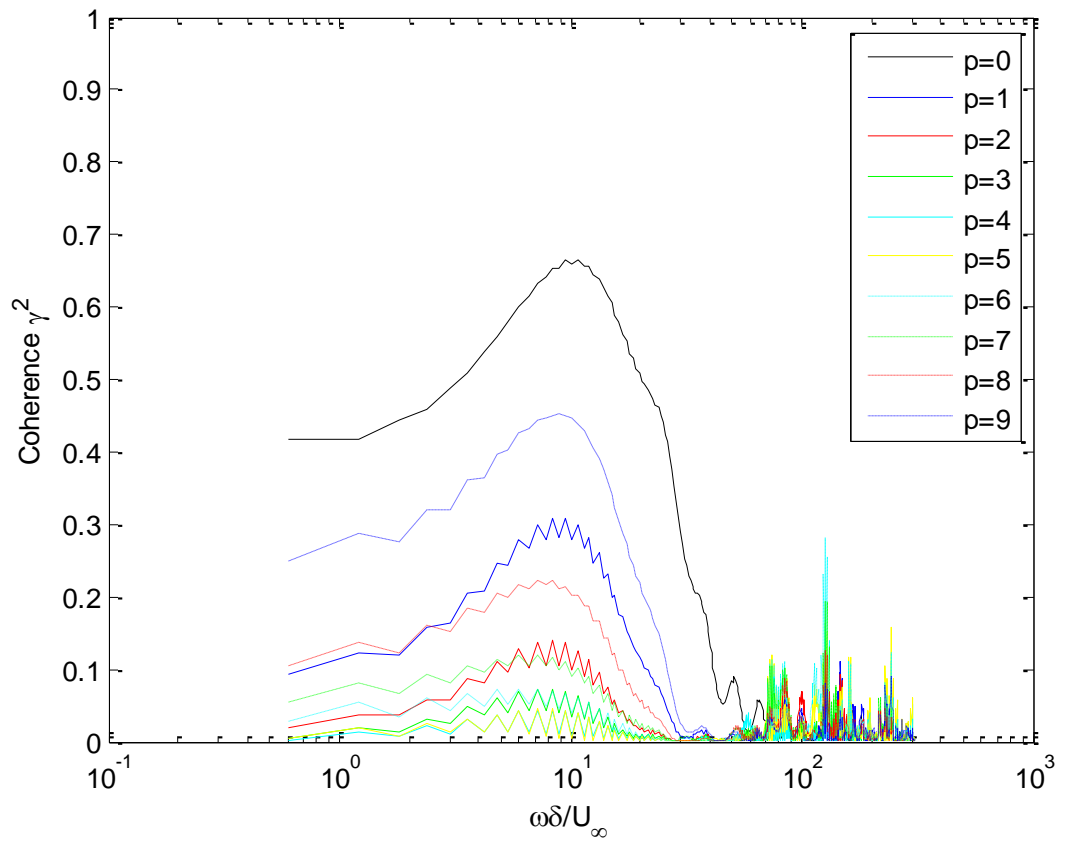

Figure 3.26. Blade to blade coherence between velocity fluctuations seen at the blade tip and at the $95 \%$ radial locations on following blades. $\left(R_{n}=1 R_{k}=0.95\right)$

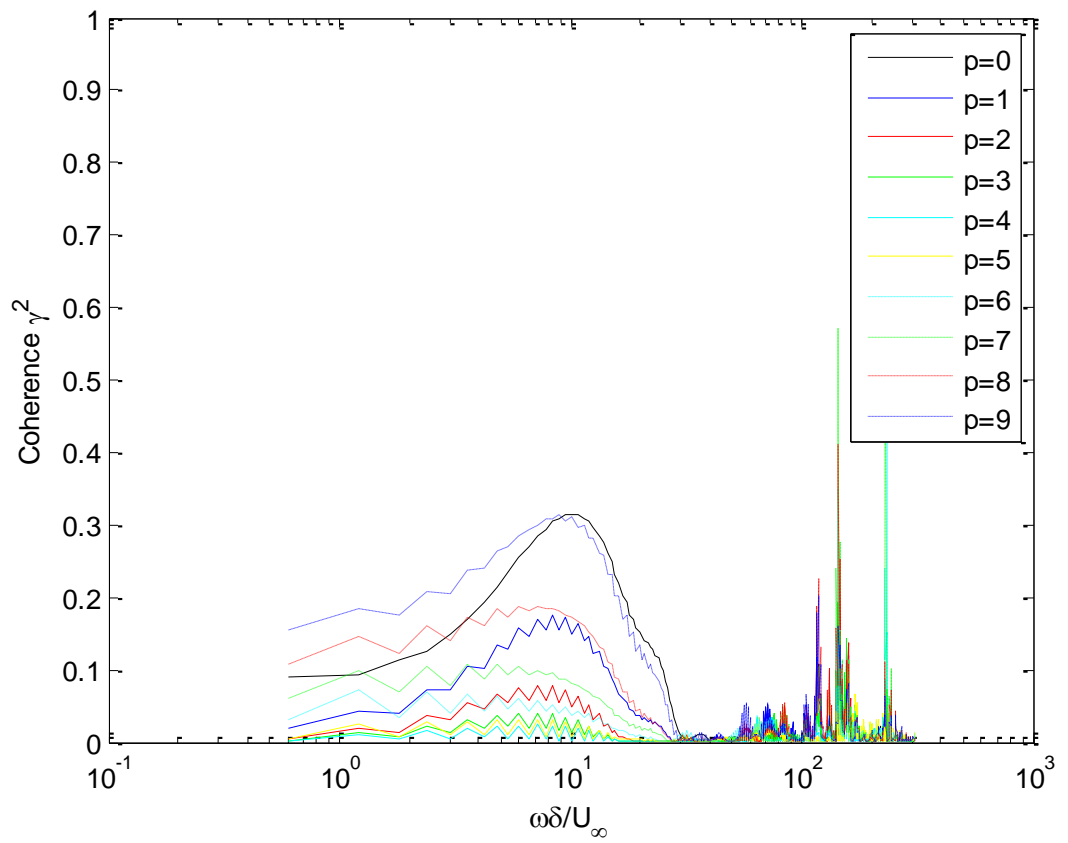

Figure 3.27. Blade to blade coherence between velocity fluctuations seen at the blade tip and at the $90 \%$ radial locations on following blades. $\left(R_{n}=1 R_{k}=0.90\right)$ 


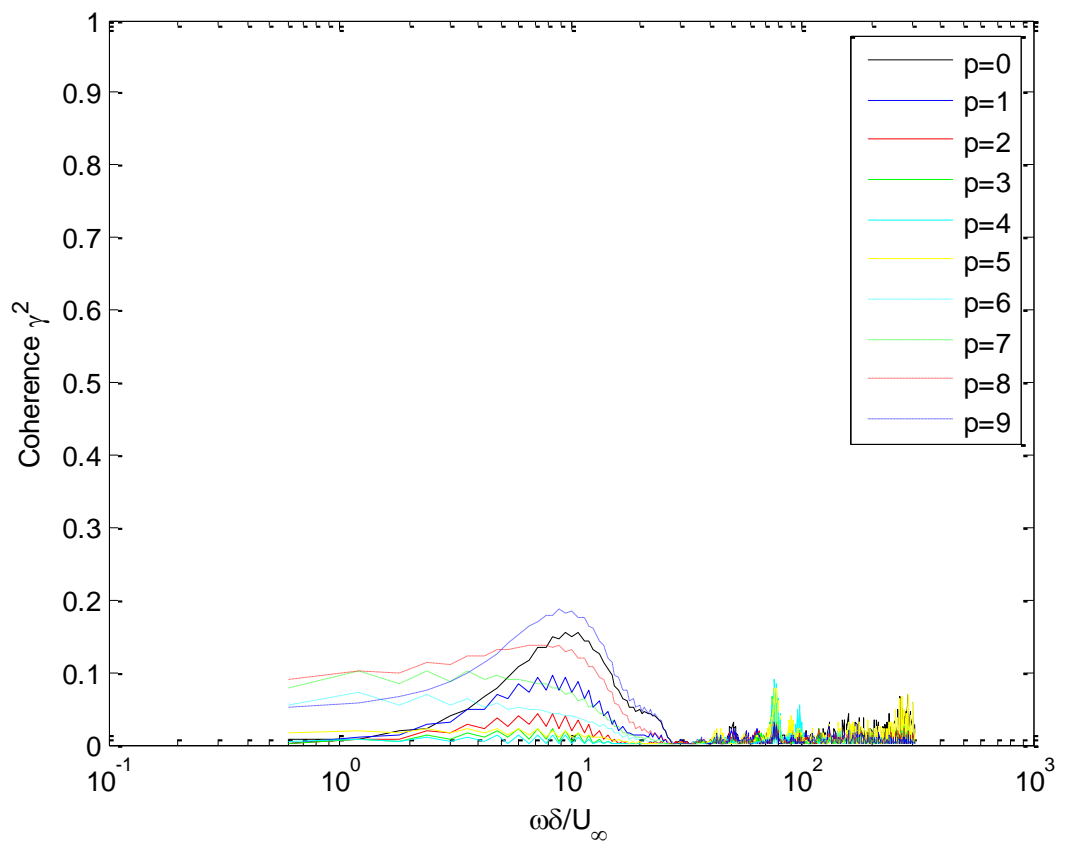

Figure 3.28. Blade to blade coherence between velocity fluctuations seen at the blade tip and at the $85 \%$ radial locations on following blades. $\left(R_{n}=1 R_{k}=0.85\right)$

\subsubsection{Farfield Sound}

Blade radii were discretized into 10 logarithmically spaced locations starting at the blade tip and decreasing to $60 \%$ of the blade radius since outside of this area the rotor blades do not produce significant sound. Source time intervals were computed exactly as discussed in Section 3.5.3. The far-field sound spectrum $S_{p p}$ was evaluated as a function of observer location $\boldsymbol{x}$ by utilizing Equation 3.14 -Equation 3.18. All noise predictions are compared to the above baseline case where design conditions consist of a free stream velocity of $20 \mathrm{~m} / \mathrm{s}$, boundary layer thickness of $.101 \mathrm{~m}$, advance ratio of 1.17 to match Sevik, and gap of $20.3 \mathrm{~mm}$ between the wall and blade tips. All results are shown as sound pressure level per frequency relative to $20 \mu \mathrm{Pa}^{2}$.

\subsubsection{Noise Predictions without Wall Effects}

By restricting the observer to a location 5 meters directly downstream on the axis of the rotor the effect of various operating and flow conditions can be analyzed. Figure 3.29 shows sound predictions of sound spectra, plotted as single-sided $1 \mathrm{~Hz}$ bandwidth SPL, for various advance ratios (J) with all other conditions remaining the same. An increase in advance ratio with free stream velocity remaining constant effectively means a decrease in rotor rpm. This decrease in rotor rpm decreases the blade passing frequency thus causing the haystacking humps to occur at lower frequencies. Also, an increase in advance ratio means blade to blade correlation in the boundary layer decreases causing the overall decibel levels to drop by $11 \mathrm{~dB}$ from an advance ratio of 0.7 to 1.44 . Looking at the theory, and ignoring the change in amplitude of the Sears function with frequency, one would expect the spectral level of the sound at the blade passing frequency to increase approximately as the 3rd power of the of the angular velocity of the rotor. Due to the frequency dependence of the upwash spectrum and the effect of the Sears function on the 
unsteady lift, the predicted change in sound level is slightly larger. An advance ratio of 1.44 represents the rotor's zero thrust condition, as predicted by JavaProp, meaning there would be no distortion present in the inflow. For $J=0.7$ the haystacking phenomenon in the sound spectrum is noticeable over 5 blade passing frequencies compared to only 4 for an advance ratio of 1.44. Since we are not accounting for distortion in the present noise predictions, results are most realistic for the $J=1.44$ case, but likely underestimate the haystacking for $J=1.17$ and 0.7 . At a free stream velocity of $20 \mathrm{~m} / \mathrm{s}$ advance ratios of 0.7 , 1.17 , and 1.44 imply rotor rpms of $3749.5,2243.3$, and 1822.69 respectively.

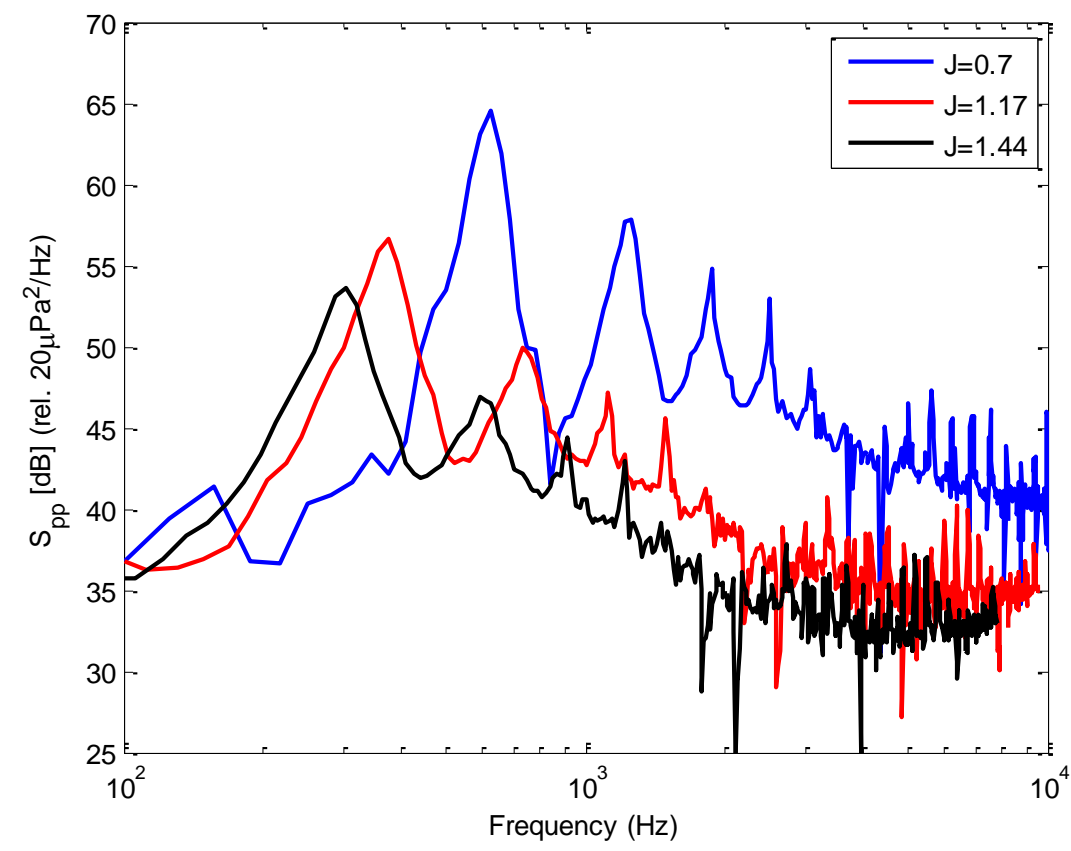

Figure 3.29. On axis sound pressure level at various advance ratios.

The amount the rotor is immersed in the boundary layer also plays a significant role in sound production. Figure 3.30 shows a comparison between 3 different rotor immersions designated by the distance the tip of the rotor blades get to the wall. The red line represents the baseline condition of a tip gap of $20.3 \mathrm{~mm}$. As shown in the figure, increasing the tip gap by a factor of 2 from the baseline condition to $40.6 \mathrm{~mm}$ decreases the sound spectrum over the entire frequency range by about $4 \mathrm{~dB}$ without changing the spectral shape. This is expected since an increase in the gap between the wall and rotor blade means less blade immersion and interaction with turbulence. Decreasing the gap to $10.15 \mathrm{~mm}$ increases the sound level at blade passing frequencies only slightly by approximately $1.5 \mathrm{~dB}$. This is due to the fact that the majority of the turbulence in the boundary layer was already interacting with the rotor blades when there was a tip gap of $20.3 \mathrm{~mm}$. Figure 3.31 shows the comparison between the design condition of $U_{\infty}=20 \mathrm{~m} / \mathrm{s}$ and $U_{\infty}=30 \mathrm{~m} / \mathrm{s}$. As expected the increase in free stream velocity increases both the sound spectrum across the frequency range and shifts the haystacking effect to higher frequencies. The shift in frequency of the haystacks occurs because in order to increase the free stream velocity and remain at the design advance ratio of 1.17 , the rotor angular velocity must be increased. Evaluating $S_{p p}$ theoretically suggests an approximate scaling of free stream velocity to the fifth power. Therefore, since the frequency scale also depends 
on $U_{\infty}$ the total change in SPL demonstrates a $U_{\infty}{ }^{4}$ dependence. Prior measurements in the Virginia Tech Stability Wind Tunnel have determined that both in flow and out of flow noise levels are below $40 \mathrm{~dB}$ at $20 \mathrm{~m} / \mathrm{s}$ meaning the effects of the rotor can be measured.

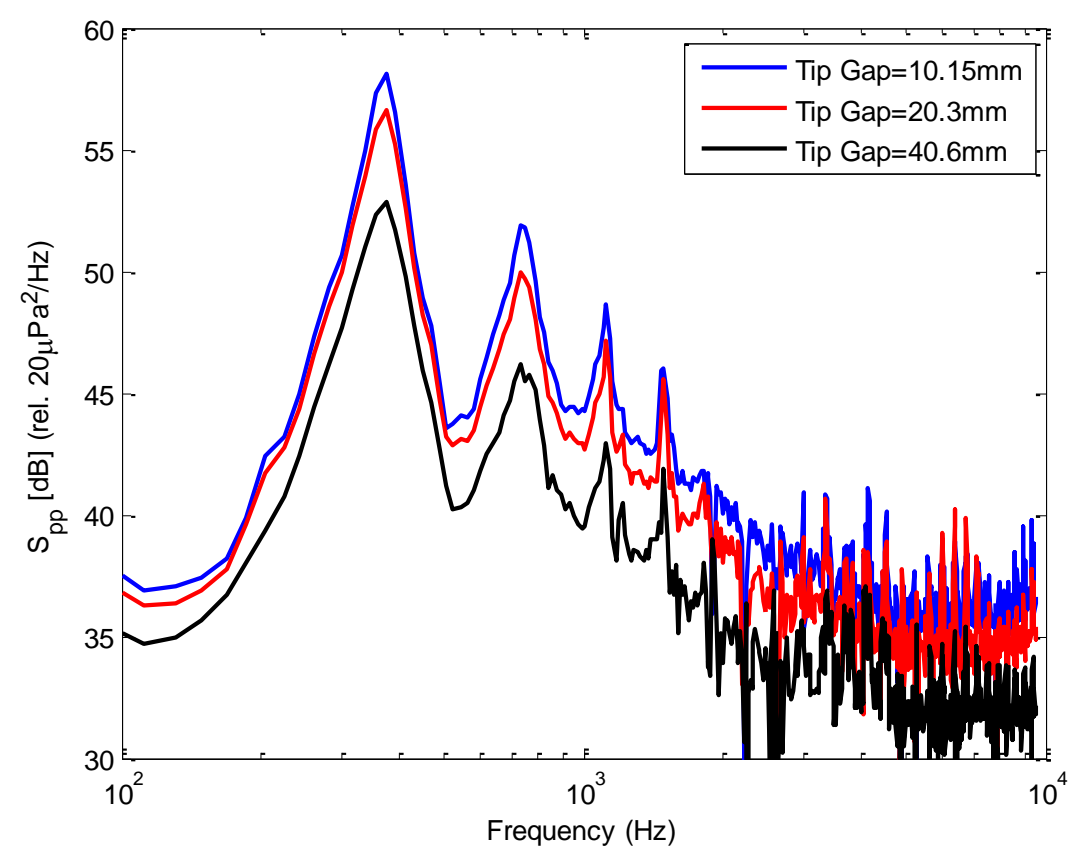

Figure 3.30. On axis sound pressure level at various tip gap heights.

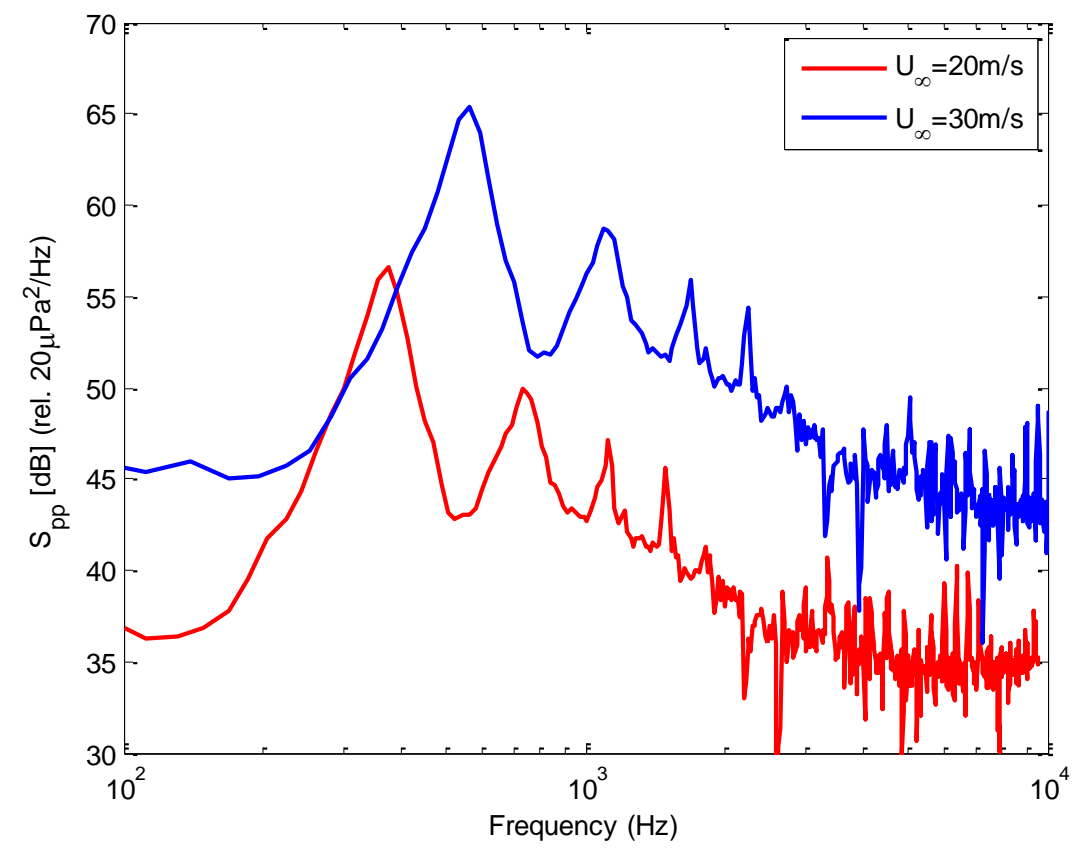

Figure 3.31. On axis sound pressure level at free stream velocities of 20 and $30 \mathrm{~m} / \mathrm{s}$. 
Due to the fact that in Equation 3.14 the unsteady lift spectrum is evaluated at a frequency of $\omega-m \Omega$ simple directivity plots at chosen frequencies do not accurately describe the far-field sound. Shown in Figure 3.32, as the angle that the observer is located about the $x_{2}$ axis increases there is a noticeable shift in spectral noise predictions especially at higher frequencies. The same shift occurs when analyzing the directivity about the $x_{3}$ axis thus it is of greater interest to plot directivity as contour plots. Figure 3.33 shows the sound pressure level about the $x_{2}$ axis for the baseline case of an advance ratio of 1.17, free stream velocity of $20 \mathrm{~m} / \mathrm{s}$, and tip gap of $20.3 \mathrm{~mm}$. In a plot like this a monopole at a given frequency would be represented by a vertical contour while a dipole would be represented by a steep gradient in contours as the 90 degree location is approached. Therefore, the farfield sound pressure level about the $x_{2}$ axis acts like a combination of a monopole and dipole. Increasing the angle from the streamwise on axis location does not seem to produce a significant effect on the overall sound level until angles greater than 30 degrees. At the 90 degrees location (in the plane of the rotor) the overall sound level is significantly less than at the on axis locations ( 0 and 180 degrees). The white regions of the plot are areas where the sound level is below the minimum $30 \mathrm{~dB}$. Figure 3.34 shows the directivity about the $x_{3}$ axis which is almost identical to the directivity about the $x_{2}$ axis as we would expect since the effect of the wall is not being considered. Analyzing the directivity about the $x_{1}$ axis with the same contour levels reveals very little since in the plane of the rotor the sound level is much less.

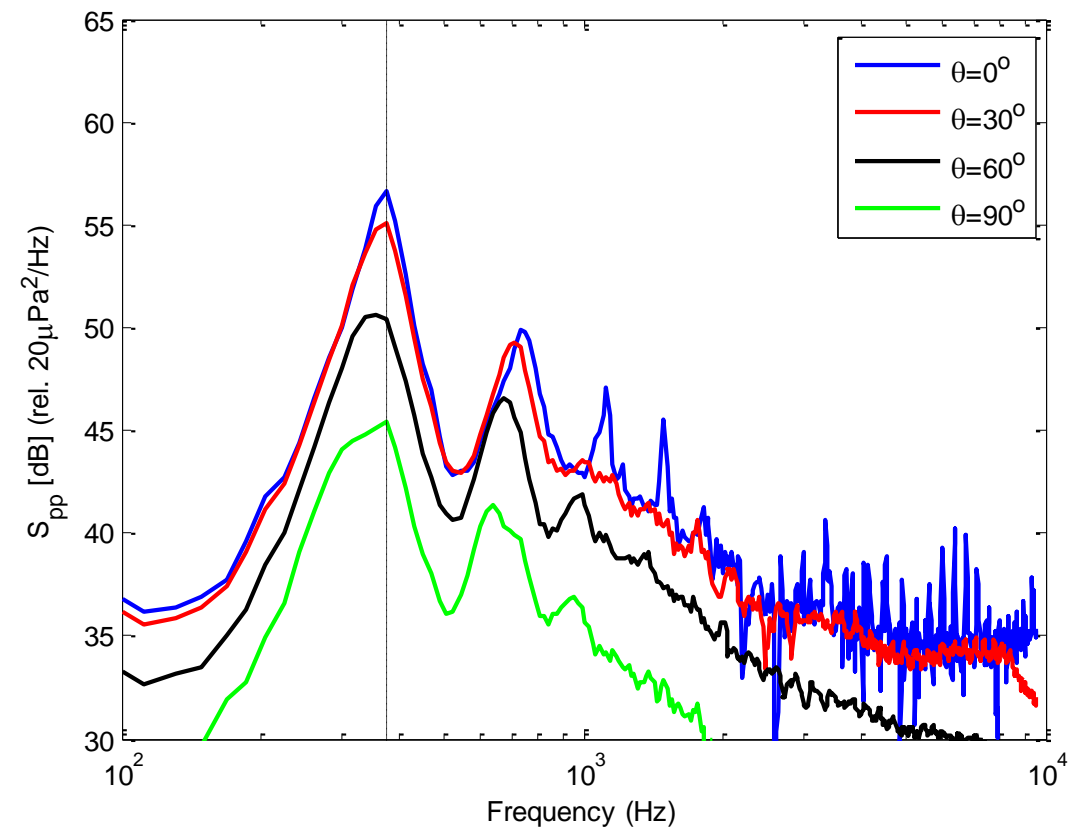

Figure 3.32. Sound pressure levels at various angles about the $x_{2}$ axis $(\phi=90)$. The dashed black line represents the first blade passing frequency. 


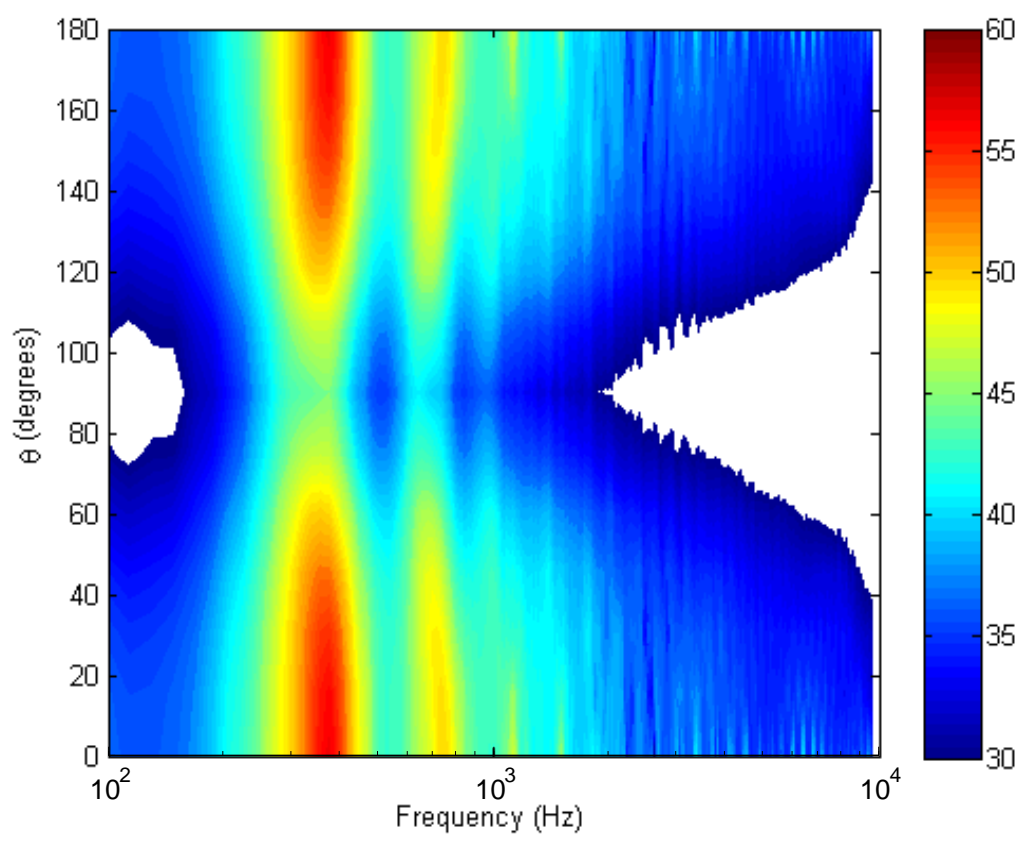

Figure 3.33. Far-field sound levels about the $x_{2}$ axis without the effect of the wall. Contour levels are $S_{p p}[\mathrm{~dB}]\left(\mathrm{rel} .20 \mu \mathrm{Pa}^{2} / \mathrm{Hz}\right)$.

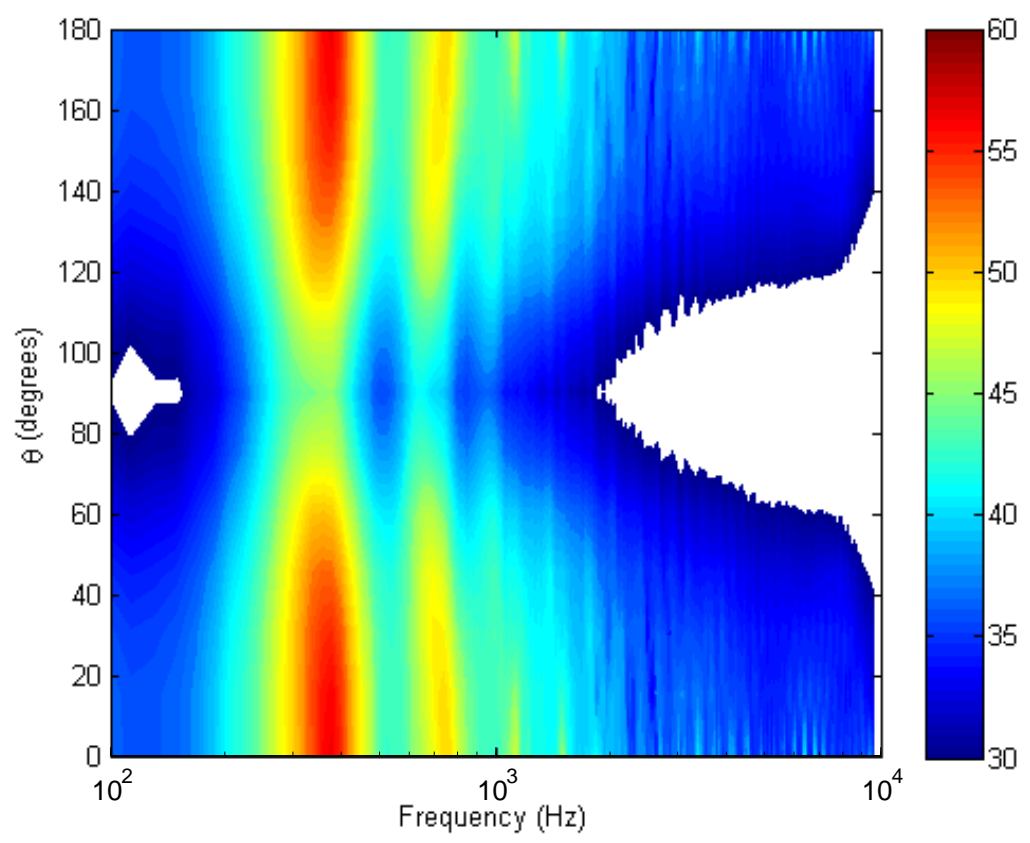

Figure 3.34. Far-field sound levels about the $x_{3}$ axis without the effect of the wall. Contour levels are $S_{p p}[\mathrm{~dB}]\left(\right.$ rel. $\left.20 \mu \mathrm{Pa}^{2} / \mathrm{Hz}\right)$.

\subsubsection{Noise Predictions with Wall Effects}

To include the effects of the wall on the far-field sound predictions the Fourier coefficients in Equation 3.14 were computed using Equation 3.19. As expected, the addition of the wall increases the overall sound levels over the entire frequency range as shown in 
Figure 3.35. This figure shows that an observer located 5 meters directly downstream on the axis of the rotor would experience a sound field $5 \mathrm{~dB}$ higher with the wall for the baseline case. A $5 \mathrm{~dB}$ increase is significant and also advantageous for future sound measurements of this configuration in the Virginia Tech Stability Wind Tunnel.

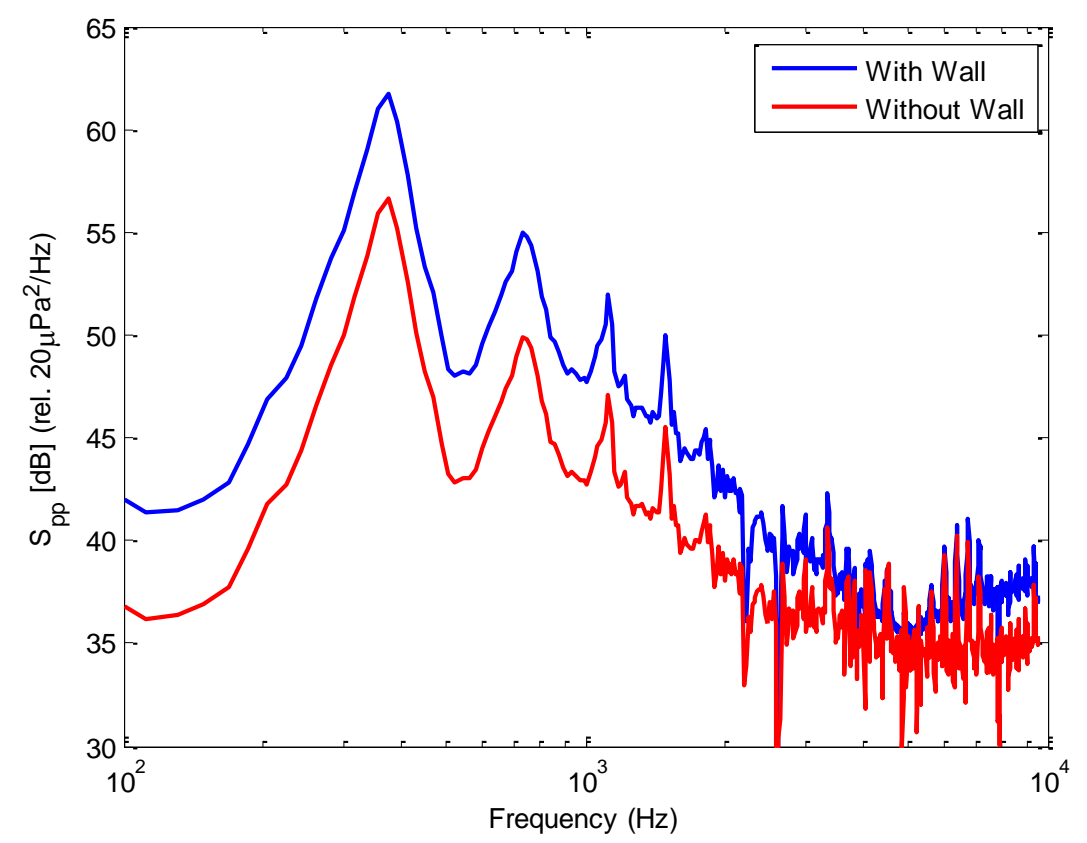

Figure 3.35. Effect of the wall on the far-field sound at an observer located $5 \mathrm{~m}$ downstream on the axis of the rotor plane for the baseline case.

Figure 3.36 shows the sound field about the $x_{2}$ axis where one can see that the wall also has a significant effect on the directivity. Not only does the wall increase the sound levels about the $x_{2}$ axis, it also increases the shift to lower frequencies as the plane of the rotor is approached. This is shown by the high $\mathrm{dB}$ lobes, represented by the first blade passing frequency, shifting to the left in the figure as the observer moves toward the 90 degree location. Also, at 90 degrees about the $x_{2}$ axis wall has little effect on the sound level. Analyzing the effect of the wall on the directivity about the $x_{3}$ axis demonstrates an increase in sound level similar to the previous figure but the shift to lower frequencies is not nearly as dramatic. Figure 3.37 shows the directivity about the $x_{3}$ axis for the baseline case. The lobes at the blade passing frequencies demonstrate nearly monopole characteristics. Observer locations close to the plane of the rotor reveal higher sound levels than about the $x_{2}$ axis since the observer is much closer to the wall when directivity is plotted about the $x_{3}$ axis. 


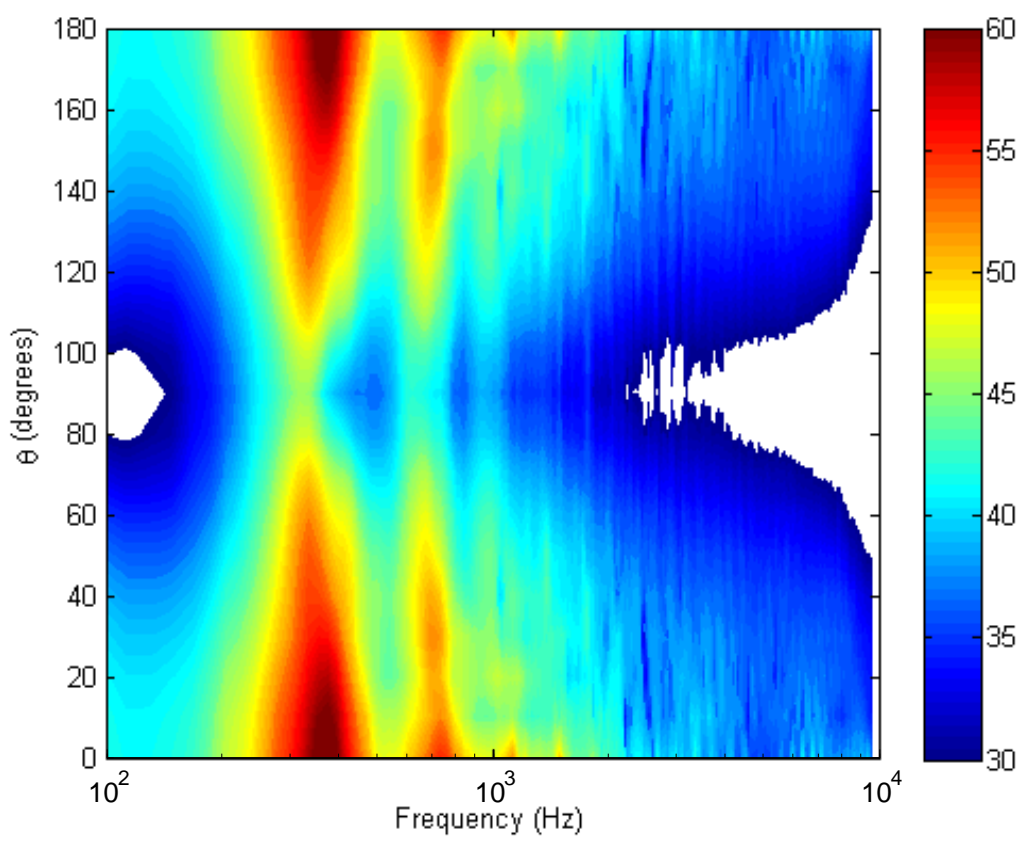

Figure 3.36. Far-field sound levels about the $x_{2}$ axis with the effect of the wall. Contour levels are $S_{p p}[\mathrm{~dB}]\left(\mathrm{rel} .20 \mu \mathrm{Pa}^{2} / \mathrm{Hz}\right)$.

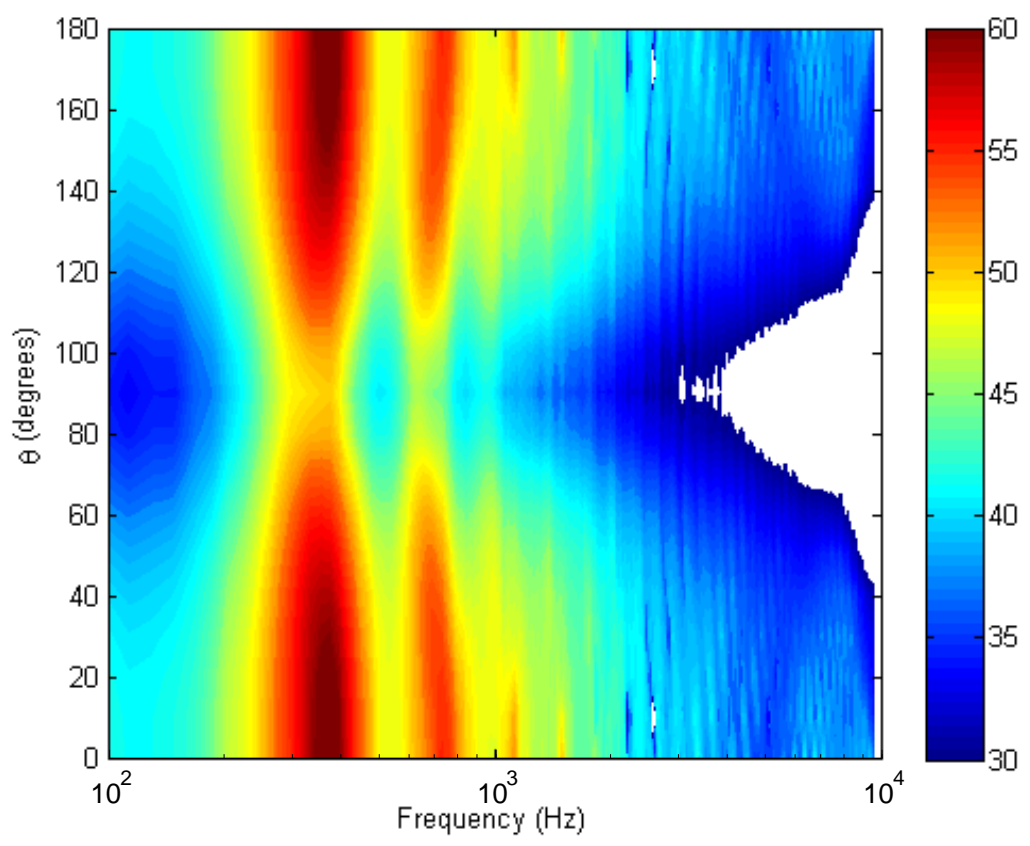

Figure 3.37. Far-field sound levels about the $x_{3}$ axis with the effect of the wall. Contour levels are $S_{p p}[\mathrm{~dB}]\left(\right.$ rel. $\left.20 \mu \mathrm{Pa}^{2} / \mathrm{Hz}\right)$. 


\section{CHAPTER 4 Conclusions}

The present study is concerned with the aerodynamics and aeroacoustics of a rotor partially immersed in a thick turbulent boundary layer. This simple configuration reconstructs the important physical processes involved when a rotor ingests a non-uniform turbulent flow, without containing many of the complexities that are common in most realworld applications. Detailed measurements of the four-dimensional space time correlation function of a thick turbulent boundary layer generated over a smooth wall have been performed and used to evaluate the source terms for rotor noise prediction using the theory of Glegg at al. (2012). The anechoic test section of the Virginia Tech Stability Wind Tunnel has been modified to include a false wall consisting of 6 Lexan panels over which the thick, high Reynolds number turbulent boundary layer flows. Single point boundary layer measurements reveal profiles typical of a fully developed zero pressure gradient twodimensional boundary layer which has a thickness of $101 \mathrm{~mm}$ and momentum thickness Reynolds number of 16,607 at a free stream velocity of $30 \mathrm{~m} / \mathrm{s}$. Two-point measurements were performed over a cross section of the flow under these conditions with measurement grids designed using space time correlation estimates based on channel flow simulations by Moser et al. (1999). When fully combined, these grids included over 1800 different relative positions in the boundary layer with a resolution of $4 \%$ of the boundary layer thickness. This allowed for the full four dimensional space time correlation function to be determined which is used to evaluate the source terms seen by the rotor. Analyzing the correlation function reveals anisotropy in the flow mainly showing a large scale correlation structure elongated in the streamwise direction and inclined about 30 degrees from the wall. One would expect this directionality in the correlation to have a substantial effect on the timing of blade to blade lift correlations seen by a cutting rotor, and thus the radiated sound.

A theory for the sound radiated from unsteady loading on a rotor due to blades cutting inhomogeneous turbulence has been previously developed by Glegg et al (2012). Using this theory and the measured turbulence data in the flow, noise predictions were performed for a 2.25 scaled version of the Sevik rotor. This is a $457.2 \mathrm{~mm}$ diameter rotor consisted of 10 unskewed constant chord blades. The correlation and cross spectrum of upwash velocity fluctuations seen in the blade frame of reference was determined from the measured data as a requirement for the theory of Glegg et al. Correlations between the first pass of a blade tip with the second are seen, a direct consequence of the large size of the boundary layer compared to the rotor and the long streamwise scales of the turbulence. Crossspectra along the radius of a blade show significant coherence over 80 percent of the blade which corresponds to just fewer than half the thickness of the boundary layer. Blade to blade coherence show asymmetry in the upwash that results from the inclination of the boundary layer structures relative to the wall which produce a correlation between velocity fluctuations in the upper part of the boundary layer and fluctuations seen at a later time close to the wall.

Far-field sound predictions were performed for a range of conditions involving advance ratio, rotor immersion depth, and free stream velocity. Sound spectra are first discussed in detail without considering the effect of the wall on sound propagation. These calculations reveal broadband sound with the presence of "haystacking" which occurs at multiples of the blade passing frequency. Sound levels were strongly dependent on flow 
and operating conditions as evident in the $11 \mathrm{~dB}$ increase when advance ratio is decreased from 1.44 to 0.7 . Directivity predictions reveal the far-field sound pressure level acts like a combination of a monopole and dipole. However, there is a left shift in frequency present when the observer approaches the plane of the rotor. Including the effects of the wall on sound propagation increases the overall sound levels by approximately $5 \mathrm{~dB}$ and increases the shift to lower frequencies as the rotor plane is approached. Overall, sound levels are predicted to be higher than the background noise of the Virginia Tech Stability Wind Tunnel allowing physical measurements to be made where the haystacking effect should be enhanced due to the stretching of turbulence as it is drawn into the rotor - an effect neglected in the present calculations. 


\section{REFERENCES}

Atassi, H., and M. Logue. "Fan Broadband Noise in Anisotropic Turbulence." 15th AIAA/CEAS Aeroacoustics Conference. Miami, FL, 2009.

Awasthi, M. High Reynolds Number Turbulent Boundary Layer Flow over Forward Facing Steps. Thesis, Virginia Tech, 2012.

Blake, W. Mechanics of Flow Induced Sound and Vibration. New York: Wiley, 1986.

Borgoltz, A. Modifications of Coherent Structures in Fan Blade Wakes for Broadband Noise Reduction. PhD Dissertation, Virginia Tech, 2007.

Catlett, M. R., J. M. Anderson, and D. O. Stewart. "Aeroacoustic Response of Propellers to Sheared Turbulent Inflows." 18th AIAA/CEAS Aeroacoustics Conference. Colorado Springs, CO, 2012.

de la Rosa Blanco, E., C. A. Hall, and D. Crichton. "Challenges in the Silent Aircraft Engine Design." 45th AIAA Aerospace Sciences Meeting and Exhibit. Reno, Nevada, 2007.

Devenport, W. J., C. W. Wenger, S. A. Glegg, and J. A. Miranda. "Wavenumber Frequency Spectra in a Lifting Wake for Broadband Noise Prediction." AIAA Journal, 1998: vol. 36, no. 6, pp 881-887.

Devenport, W. J., R. Burdisso, A. Borgoltz, R. Patricio, and M. Barone. "Aerodynamic and Acoustic Corrections for a Kevlar-Walled Anechoic Wind Tunnel." 16th AIAA/CEAS Aeroacoustics Conference. Stockholm, Sweden, 2010. AIAA-2010-3749.

Forest, J. B. The Wall Pressure Spectrum of High Reynolds Number Rough-Wall Turbulent Boundary Layers. M.S. Thesis, Blacksburg, Virginia: Aerospace and Ocean Engineering Department, Virginia Tech, 2012.

Ganz, U., P. Joppa, T. Patten, and D. Sharpf. Boeing 18-inch Fan Rig Broadband Noise Test. NASA, 1998.

Gavin, J. R., and G. C. Lauchle. "Modeling the Timespace Correlations in the Wake Region of a Turbulent Boundary Layer." Proceedings of ASME Winter Meeting. Orlando, FL, 2000.

Glegg, S. A., and W. J. Devenport. "Proper Orthogonal Decomposition of Turbulent Flows for Aeroacoustic and Hydroacoustic Applications." Journal of Sound and Vibration, 2001: vol. 239, no. 4, pp. 767-784.

Glegg, S. A., M. A. Morton, and W. J. Devenport. "Rotor Inflow Noise Caused by a Boundary Layer: Theory and Examples." 18th AIAA/CEAS Aeroacoustics Conference. Colorado Springs, CO, 2012.

Glegg, S., and N. Walker. "Fan Noise from Blades Moving Through Boundary Layer Turbulence." 5th AIAA/CEAS Aeroacoustics Conference. Bellevue, WA, 1999.

Graham, J. M. R. "The Effect of a Two-Dimensional Cascade of Thin Streamwise Plates on Homogeneous Turbulence." Journal of Fluid Mechanics, 1998: 125-147.

Hanson, D. "Spectrum of Rotor Noise Caused by Atmospheric Turbulence." Journal of the Acoustical Society of America, 1974: 110-126.

Hileman, J. I., Z. S. Spakowsky, M. Drela, and M. A. Sargeant. "Airframe design for silent aircraft." 45th AIAA Aerospace Sciences Meeting and Exhibit. Reno, Nevada, 2007. 453.

Huyer, S., and D. Beal. "A Turbulent Inflow Model Based on Velocity Modulation." Journal of Sound and Vibration, 2007: vol. 308, pp. 28-43. 
Huyer, S., and S. Snarski. "Analysis of a Turbulent Propeller Inflow." Journal of Fluids Engineering, 2003: vol. 125, pp. 533-542.

Joseph, P., and A. Parry. "Rotor/Wall Boundary-Layer Interaction Broadband Noise in Turbofan Engines." 7th AIAA/CEAS Aeroacoustics Conference. Maastricht, Netherlands, 2001.

Ko, A., J. Schetz, and W. Mason. "Assessment of the Potential Advantages of DistributedPropulsion for Aircraft." XVI International Symposium on Air Breathing Engines (ISABE). Cleveland, Ohio, 2003.

Lumley, J. L. "The Structure of Inhomogeneous Turbulence." Atmospheric Turbulence and Wave Propagation, 1967: pp. 166-178.

Lynch III, D. A., W. K. Blake, and T. J. Mueller. "Turbulence Correlation Length-scale Relationships for the Prediction of Aeroacoustic Response." AIAA Journal, 2005: 1187-1197.

Lynch III, D. A., W. K. Blake, and T. J. Mueller. "Turbulent Flow Downstream of a Propeller, Part 2: Ingested, Propeller-Modified Turbulence." AIAA Journal, 2005: 1211-1220.

Lynch III, D. A., W. K. Blake, and T. J. Mueller. "Turbulent Inflow Downstream of a Propeller, Part 1: Wake Turbulence." AIAA Journal, 2005: 1198-1210.

Majumdar, S., and N. Peake. "Noise Generation by the Interaction Between Ingested Turbulence and a Rotating Fan." Journal of Fluid Mechanics, 1998: 181-216.

Martinez, R. "Broadband Sources of Structure-Borne Noise for Propulsors in "Haystacked" Turbulence." Computers and Structures, 1997: 475-490.

Minniti, R. J., W. K. Blake, and T. J. Mueller. "Inferring Propeller Inflow and Radiation from Near-Field Response, Part 1: Analytic Development." AIAA Journal, 2001: 10371046.

Minniti, R. J., W. K. Blake, and T. J. Mueller. "Inferring Propeller Inflow and Radiation from Near-Field Response, Part 2: Empirical Application." AIAA Journal, 2001: 1030-1036.

Moiseev, N., B. Lakshminarayana, and D. Thompson. "Noise Due to Interaction of Boundary Layer Turbulence with a Compressor Rotor." Journal of Aircraft, 1978: vol. 15, pp. 53-61.

Moser, R. B., J. Kim, and N. N. Mansour. "Direct numerical simulation of turbulent channel flow up to Ret=590." Physics of Fluids, 1999: Vol. 11, Number 4, pp. 943-945.

Muench, J. Periodic Acoustic Radiation from a Low Aspect Ratio Propeller. PhD Dissertation, University of Rhode Island, 2001.

Plas, A. P. Performance of a Boundary Layer Ingesting Propulsion System. Thesis, Massachusetts Institute of Technology, 2006.

Pope, S. B. Turbulent Flows. Cambridge, UK: Cambridge University Press, 2000.

Remillieux, M. C., et al. "Calibration and Demonstration of the New Virginia Tech Anechoic Wind Tunnel." 14th AIAA/CEAS Aeroacoustics Conference. Vancouver, British Columbia, May 5-7, 2008. AIAA-2008-2911.

Scharpf, D. F., and T. J. Mueller. "An Experimental Investigation of the Sources of Propeller Noise due to the Ingestion of Turbulence at Low Speeds." Experiments in Fluids 18, 1995: 277-287.

Sevik, M. "Sound Radiation from a Subsonic Rotor Subjected to Turbulence." Symposium on Fluid Mechanics, Acoustics and Design of Turbomachinery. Pennsylvania State University, 1970. 
Stephens, D., and C. Morris. "Sound Generation by a Rotor Interacting with a Casing Turbulent Boundary Layer." AIAA Journal, 2009: 2698-2708.

Wittmer, K. S., W. J. Devenport, and J. S. Zsoldos. "A four-sensor hot-wire probe system for three-component velocity measurement." Experiments in Fluids, 1998: Vol. 24, pp. 416-423.

Wojno, J. P., T. J. Mueller, and W. K. Blake. "Turbulence Ingestion Noise, Part 1: Experimental Characterization of Grid-Generated Turbulence." AIAA Journal, 2002: 16-25.

Wojno, J. P., T. J. Mueller, and W. K. Blake. "Turbulence Ingestion Noise, Part 2: Rotor Aeroacoustic Response to Grid-Generated Turbulence." AIAA Journal, 2002: 26-32. 